\title{
Both Fallopian Tube and Ovarian Surface Epithelium Can Act as Cell-of-Origin for High Grade Serous Ovarian Carcinoma
}

Shuang Zhang", Tao Zhang, Igor Dolgalev, Hao Ran, Douglas A. Levine, Benjamin G. Neel ${ }^{\#}$

Laura and Isaac Perlmutter Cancer Center, NYU Langone Health, New York, NY, 10016, USA.

${ }^{\#}$ Corresponding authors: Benjamin G. Neel, New York University School of Medicine, 522 First Avenue, Smilow Building $12^{\text {th }}$ Floor, Suite 1201, New York, NY 10016. Phone: 212-263-3019; Fax: 212-263-9190; E-mail:Benjamin.Neel@nyumc.org; Shuang.Zhang@,nyumc.org

Running title: Cell-of-origin of High Grade Serous Ovarian Cancer

Keywords: high-grade serous ovarian cancer, ovarian surface epithelial cell, fallopian tube secretory epithelial cell, Pax8, Lgr5

Financial Support: This work was supported by Canadian Institutes for Health Research MOP-191992, the Department of Defense Ovarian Cancer Research Program W81XWH-15-1-0429, Ovarian Cancer Research Fund Alliance Postdoc fellowship and Cancer Center Core Grant P30 CA016087.

Conflicts of Interest: Benjamin G. Neel is co-founder, holds equity in, and received consulting fees from Navire Pharmaceuticals and Northern Biologics, Inc. 


\begin{abstract}
The cell-of-origin of high grade serous ovarian carcinoma (HGSOC) remains controversial, with fallopian tube epithelium (FTE) and ovarian surface epithelium (OSE) each suggested as candidates. Here, by using genetically engineered mouse models and novel organoid systems, we assessed the tumor-forming capacity and properties of FTE and OSE harboring the same oncogenic abnormalities. Combined RB family inactivation (via T121 expression) and Tp53 mutation in Pax8+ FTE caused transformation to Serous Tubal Intraepithelial Carcinoma (STIC), which rapidly metastasized to the ovarian surface. This mouse model was recapitulated by FTE organoids, which, upon orthotopic injection, generated widely metastatic HGSOC. The same genetic lesions in Lgr5 + OSE cells or organoids also caused metastatic HGSOC, although with longer latency and lower penetrance. Comparative transcriptome analysis was consistent with different human HGSOCs arising from FTE and OSE. Furthermore, FTE- and OSE-derived organoids showed differential sensitivity to HGSOC chemotherapeutics. Our results comport with a dualistic origin for HGSOC and suggest the cell-of-origin could influence therapeutic response.
\end{abstract}

SIGNIFICANCE: The cell-of-origin for high grade serous ovarian carcinoma (HGSOC) has been controversial. By generating novel GEMMs and organoid models with the same oncogenic defects, we demonstrate that HGSOC can originate from either fallopian tube epithelium (FTE) or ovarian surface epithelium (OSE). Importantly, FTE- and OSE-derived tumors differ significantly in biologic properties. 


\section{INTRODUCTION}

High-grade serous ovarian cancer (HGSOC) is the most common and deadly ovarian epithelial cancer, causing $\sim 70 \%$ of ovarian cancer deaths (1). Although HGSOC typically presents as a large ovarian mass accompanied by widespread peritoneal metastasis, its cell-of-origin remains controversial (2-5). Historically, as its name implies, HGSOC was thought to arise from the ovarian surface epithelium (OSE) or, more specifically, from cortical inclusion cysts, invaginations that result from normal ovulatory "wounds." "Trapped" OSE within these cysts were believed to undergo Mullerian metaplasia and accumulate causal mutations (5-7). Cortical inclusion cysts with columnar (Mullerian) epithelia and focal p53 immunoreactivity, but not frank carcinoma, have been reported, although such lesions are relatively rare $(8,9)$. More recently, attention has turned to the fallopian tube epithelium (FTE) as the cell of origin for HGSOC. Initial studies revealed lesions termed Serous Tubular Intraepithelial Carcinomas (STICs) in the fallopian tube fimbria of women with BRCA1/2 mutations undergoing riskreducing salpingoophorectomy (10-12). Subsequently, STICs were reported in up to $60 \%$ of sporadic HGSOC patients (13-15).

Molecular analyses strongly support a fallopian tube (FT) origin of most cases of HGSOC. Essentially all HGSOCs have TP53 somatic mutations, and STICs, primary tumors and HGSOC metastases have the same TP53 mutation, implying a shared origin (17-20). More comprehensive genomic characterization (e.g., exome sequencing, copy number analyses, targeted amplicon deep sequencing and gene expression profiling) has shown that, in most cases, ovarian masses and distant metastases have the same "truncal" lesions as STICs, but also possess additional, often sub-clonal, genomic abnormalities, suggesting that STICs are precursor lesions (13,21). Consistent with these findings, the transcriptome of most HGSOCs more closely resembles that of normal FTE than to OSE; nevertheless, up to $12 \%$ of HGSOCs show greater transcriptional similarity to OSE $(22,23)$. Furthermore, some genomic studies have found that HGSOC can metastasize to the FT and mimic STICs $(24,25)$, consistent with a non-FTE origin. In addition, FTs can harbor metastases that mimic STICs but arise from other sites (e.g., uterine serous carcinoma) (25-28). The Mullerian features of HGSOC have been cited as evidence of a FT origin, as FT is a Mullerian-derived structure. However, OSE and FTE share an even earlier embryonic progenitor $(6,29,30)$, and inappropriate expression of $H O X$ family members can confer Mullerian-like features in mouse models (31).

Mouse modeling has also failed to definitively define the cell-of-origin for HGSOC. Lineage tracing identified a stem cell niche in the mouse ovarian hilum containing Lgr $5+$ cells that can give rise to the entire OSE, and it was suggested that these cells were the target of transformation in HGSOC pathogenesis (32). HGSOC-like tumors have been generated by injecting adenovirus expressing Cre recombinase (Ad-Cre) into the ovarian bursae of mice bearing conditional alleles of tumor suppressor genes and/or oncogenes, including Myc;Tp53;Brcal (33), Pten;Pik3ca (34), Tp53;Rb1 (35), or TgK18GT 121 (N-terminal 121 amino acids of SV40 T antigen (T121) under the control of the keratin 18 promoter) and flTp53, \pm flBrcal (36). These reports concluded that HGSOC arises from OSE, but they did not exclude the possibility that FTE cells also were infected by Ad-Cre. Conversely, a mouse model with Pten deletion, Tp53 deletion or mutation, and Brcal/2 deletion driven by the Pax 8 promoter, which is expressed selectively in FTE secretory cells (not in OSE) develops STIC-like lesions and eventually, widely metastatic HGSOC (37). Similar results were seen with in mice that express SV40 large T-antigen (TAg) (38) or with simultaneous mutation of Brcal, Tp53, Rb1 and Nf1 in Ovgpl-expressing secretory FTE (39).

In other neoplasms, the cell-of-origin can contribute to inter-tumor heterogeneity and distinct tumor biology (40-42). Therefore, we used lineage-specific Cre recombinase (Cre) lines, combined with FTE- and OSE-derived 
organoids, to ask whether introducing the same genetic defects into FTE or OSE results in HGSOC, and if so, whether the resultant tumors have similar properties.

\section{RESULTS}

\section{Perturbing Tp53 and the RB family in Pax8+ cells cause STIC and metastasis}

TgK18GT121 (hereafter, “T121”) mice harbor a bacterial artificial chromosome (BAC) containing a Creconditional loxP-GFP-stop-loxP (LSL) T121 cassette inserted into the mouse cytokeratin (CK) 18 gene. Inducing expression of T121 (which inactivates all RB family members) by bursal injection of Ad-Cre, when combined with $T p 53$ deletion or heterozygotic $T p 53^{R 172 H}$ expression, results in HGSOC at a frequency of $70-90 \%$ (36). These findings were interpreted as showing that OSE gives rise to HGSOC. However, Ad-Cre injection into mice with a Cre-inducible $l a c Z$ allele (Rosa26-lacZ) resulted in mosaic LacZ staining in FTE in addition to the expected strong OSE staining (Supplementary Fig. S1), making the actual cell-of-origin in this model unclear. We therefore investigated the consequences of introducing the same ovarian cancer-associated genetic abnormalities into either FTE or OSE alone.

To this end, we generated $T 121 ; T p 53^{R 172 H}$ mice co-expressing appropriate lineage-specific Cre constructs. Pax8rtTA mice reportedly enable doxycycline (Dox)-inducible gene expression in secretory FTE (37). To confirm the lineage fidelity of this line, we generated Pax8rtTA;TetOCre:Rosa26-tdTomato reporter mice and Pax8rtTA;TetOCre:Rosa26-Lacz reporter mice (Fig. 1A, Supplementary Figure 2). Following Dox administration $(0.2 \mathrm{mg} / \mathrm{ml}$ in drinking water ad libitum $)$ for 1 week, histologic analysis revealed strong $\beta$-gal reactivity in the FTE, but no staining in the ovary, including the OSE (Fig. 1B). We also generated Pax8rtTA;TetOcre;Rosa26-tdTomato mice and exposed them to Dox for 2 days, followed by a "chase" in Dox-free water. At Day 2, Tomato+ cells colocalized with PAX8+ secretory FTE cells, as expected (Supplementary Fig. 2A). However, after 2 months of Doxfree "chase", secretory cells (PAX8+) and ciliated cells (which stain positive for acetylated $\alpha$-tubulin) both were Tomato+ (Supplementary Fig. S2B). These data confirm that PAX8+ cells give rise to ciliated cells in vivo, consistent with a recent study (43).

Having confirmed the selective expression of Pax8rtTA in FTE, we generated Pax8rtTA;TetOcre;Tp53 $3^{R 172 H / f l}$ (hereafter, "PTP mice"), Pax8rtTA;TetOcre;T121 mice (hereafter, "PTT mice"), which direct inducible expression of T121 alone in Pax8+ cells, and Pax8rtTA;TetOcre;Tp53 ${ }^{\text {R172H/fl }}$; T121 mice (hereafter, "PTPT mice"), which enable Dox-dependent expression of T121 and deletion of one Tp53 allele on a background of $T p 53^{R 172 H}$ heterozygosity. As noted above, T121 mice contain a GFP reporter embedded within their LSL cassette (Fig. 1C), which allows deletion efficiency to be monitored by absence of GFP. As expected, in the absence of Dox, GFP was detected diffusely in FTE, which exhibited very low levels of proliferation, as shown by Ki67 staining. Following Dox administration, the newly generated GFP- cells showed substantial Ki67 positivity, indicating that T121 evokes FTE proliferation (Fig. 1D). Within 1 month, FTE in Dox-activated PTPT mice showed clear evidence of transformation, with characteristic features of STIC, including secretory cell proliferation, loss of polarity, cellular atypia, and serous histology (Fig. 1E and F). As early as 1 month post-Dox, metastases were detected on the ovarian surface (Fig. 1G, compare boxed regions). Notably, 5/9 PTT mice underwent similar FTE transformation, suggesting that RB family inactivation alone can promote STIC. This finding is consistent with a previous study in which expressing SV40 large T antigen under control of the Ovgp-1 promoter resulted in STIC 
(38). We did not detect STIC in PTP mice (0/9), but combining Tp53 perturbation with T121 expression significantly increased FTE transformation (from 55\% to 100\%) and metastasis (from 0 to $88 \%$ ) to the ovary (Fig. $1 \mathrm{~F}$ and $\mathrm{H}$ ). Immunohistochemical analysis (IHC) showed HGSOC-related features, including TP53 accumulation, proliferation and PAX8 expression in the primary lesion and the ovarian metastases of PTPT mice (Fig. 1I and J).

Unexpectedly, PTT and PTPT mice died within 2 months (Supplementary Fig. S3A), but their demise was not due to peritoneal masses/obstruction, as would be expected with lethal HGSOC. Instead, these mice had markedly enlarged thymi, which led to breathing difficulties and ultimately, respiratory arrest (Supplementary Fig. $\mathrm{S} 3 \mathrm{~B}$ ). Combined Rb/p130 deletion/p107 heterozygosity causes a similar phenotype (44), and surprisingly, reanalysis of Pax8rTtA;TetO-Cre; Rosa 26-tdTomato mice showed that TetO-Cre is active in thymic epithelial cells (although not in FTE) even in the absence of Dox (Supplementary Fig. S3C). This unanticipated "leakiness" of $\mathrm{TetO}-\mathrm{Cre}$ in the thymic epithelium precluded us from asking if Pax8-driven T121 expression/Tp53 perturbation leads to widespread metastasis, although the early ovarian studding and our organoid studies (see below) make this highly likely.

\section{FTE organoids recapitulate fallopian tube differentiation and transformation in vitro}

Organoids initiated from adult stem cells can often be propagated long-term and are useful for cancer modeling $(45,46)$. Culture conditions for human FTE organoids have been established (47), but OSE organoids have not been reported, nor have human or mouse organoids been used to model HGSOC. We developed serumfree defined media that allow mouse FTE and OSE (see below) organoids to be passaged indefinitely ( $>30$ passages) in Matrigel-based media, cryopreserved, and re-established in culture (see Methods). By 48hr after seeding FACSpurified EPCAM-/CD45- cells into such media, small (1-5 $\mu \mathrm{m}$ in diameter), round, cystic spheres appeared (EPCAM+CD45-), and they expanded rapidly to form large (100-1000 $\mu \mathrm{m}$ in diameter) hollow cysts. After 10 days, epithelial invagination resulted in mucosal folds, morphologically recapitulating FT architecture (Fig. 2A). Organoids cultured longer than 7 days contained secretory and ciliated cells, like their tissue of origin (Fig. 2B).

To evaluate the utility of this system for modeling HGSOC, we established organoid cultures from FTE of wild type, PTP, PTT, and PTPT mice. Dox-treated PTT and PTPT organoids activated Cre expression, as indicated by loss of GFP after 3 passages (Fig. 2C and data not shown). Dox-treated (but not control) PTPT organoids exhibited frank dysplasia, TP53 accumulation, and staining for $\gamma \mathrm{H} 2 \mathrm{AX}$, a marker of DNA damage (Fig. 2D). Consistent with our GEMM results (Fig. 1), PTT and PTPT organoids proliferated more rapidly and were larger than controls (Fig. 2E and F, Supplementary Fig. S4A and B). By contrast, organoid-forming efficiency was not increased in mutants, suggesting that $T p 53$ mutation and/or RB family inactivation did not affect FTE self-renewal, as least under our culture conditions (Supplementary Fig. S4C). STIC and HGSOC typically are PAX8+ (although staining can be mosaic), and they do not express ciliated cell markers (48). Similarly, ciliated cells were undetectable in PTT or PTPT organoids, suggesting that the RB family inactivation restricts FTE differentiation to the secretory lineage (Fig. 2G and H). Tp $53^{R I 72 H /-}$ mutation did not impair differentiation, but it enhanced invasive capability (Fig. 2I and J). Thus, FTE organoids can be used to deconstruct various aspects of the malignant phenotype and attribute each to specific genetic defects.

\section{FTE-derived organoids can give rise to HGSOC}


We previously found that primary human HGSOC cells implanted into the mouse mammary fat pad (MFP) recapitulate HGSOC histomorphology, inter- and intra-tumor heterogeneity, and patient drug response $(49,50)$. Therefore, as an initial test of their tumor-forming capacity, we injected wild type and mutant organoids into the MFPs of $n u / n u$ mice (Fig. 3A). Wild type and $T p 53^{R 172 H /-}$ organoids formed glandular structures composed of simple cuboidal cells that persisted for at least 4 weeks, but these disappeared ( $n=0 / 8$ for wild type, $n=0 / 9$ for PTP organoids) by 2 months (Fig. 3B-D). By contrast, PTPT organoids formed easily palpable $(>2 \mathrm{~mm})$ masses of high grade, poorly differentiated adenocarcinoma with prominent cellular/nuclear pleomorphism and frequent mitoses (Fig. 3D). PTT organoids also gave rise to HGSOC in 3 out of 8 mice, but consistent with our finding that biallelic Tp53 perturbation is associated with disease progression (Fig. 1F and H), PTPT organoids were more tumorigenic (7 out of 8 mice, Fig. 3B). The ovary is thought to provide trophic signals that enable HGSOC growth and metastasis (51), so we also assessed tumorigenicity of organoids injected into the ovarian fat pad. PTT and PTPT organoids both gave rise to primary tumors, but within 3 months, 5/7 mice injected with PTPT organoids developed ascites, sometimes hemorrhagic, with widespread peritoneal studding similar to the human disease. By contrast, 0/7 lines of PTT-derived organoids showed evident metastasis at 9 months post-injection (Fig. 3E and F). Metastases expressed HGSOC markers, including PAX8, p53, Ki67, $\gamma \mathrm{H} 2 \mathrm{AX}$, Stathmin1 and p16 (Fig. 3G). Hence, our FTE organoid system recapitulates HGSOC biology morphologically and molecularly.

\section{OSE also can give rise to HGSOC}

Ad-Cre injection into the ovarian bursa of $T p 53^{R 172 H / f l}$;T121 mice results in HGSOC (36), but as shown above, FTE cells also are infected under these conditions. As an initial test of whether OSE itself could serve as the HGSOC cell-of-origin, we performed salpingectomies 3 days after Ad-Cre injection into Tp $53^{R 172 H / f l}$;T121 mice (Supplementary Fig. S5A). Gross inspection and histology of the ovaries of injected mice confirmed that the FT had been removed (Supplementary Fig. S5B). Nevertheless, when mice were examined 3 months later, the OSE showed abnormal morphology and hyperproliferation (Supplementary Fig. S5C), which prompted us to more carefully evaluate OSE as a potential cell-of-origin for HGSOC.

Lgr5+ embryonic and neonatal populations establish the OSE lineage and the fimbrial FTE (52). In adult mice, however, Lgr5 expression is concentrated in the ovarian hilum, together with the stem cell markers CD133 and $\mathrm{ALDH}$, and lineage-tracing using $\operatorname{Lgr} 5 \mathrm{Cr} e^{E R T 2}$ mice showed that $\operatorname{Lgr} 5+$ cells can repopulate the entire OSE (32). To confirm the lineage fidelity of this Cre line, and in particular, to assess its expression in adult FTE, we

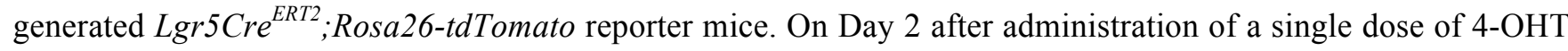
(to induce CRE activity), there were sparse Tomato+ cells in OSE. By 4 months, though, these cells had expanded and populated a significant fraction of OSE. By contrast, there was no FTE expression at either time (Fig. 4A and B).

We next generated Lgr5-Cre; Tp53 ${ }^{R 172 H / f l} ; T 121$ (LPT) mice, and compared them with Lgr5-Cre; $T p 53^{R 172 H / f l}$ (LP) and Lgr5-Cre;T121 (LT) mice, respectively (Fig. 4C). All mice received a single intraperitoneal dose of 4-OHT. As expected, LP mice $(n=0 / 10)$ did not develop tumors, and only 1 out of 12 LT mice showed an obvious ovarian mass by 18 months after 4-OHT treatment. By contrast, all (11/11) LPT mice had large, palpable, abdominal masses by 11 months post-4-OHT injection (Fig. 4D and E). Gross inspection revealed markedly enlarged, hemorrhagic ovaries, with 16\% $(\mathrm{n}=2 / 11)$ developing multifocal peritoneal carcinomatosis and ascites (Fig. 4D and F). Histology and IHC revealed poorly differentiated adenocarcinoma with papillary and 
micropapillary/filigree morphology (Fig. 4G), resembling human HGSOC. Notably, however, these tumors showed little PAX8 staining.

As an additional test of whether Lgr5+ OSE cells directly evoke HGSOC transformation, we generated compound LPT;Rosa 26-tdTomato mice (Supplementary Fig. 6A), administered a single dose of 4-OHT, and "chased" for 3, 6, or 9 months (Supplementary Fig. 6B). By 3 months following CRE activation, neoplasia had developed from $\operatorname{Lgr} 5+$ cells (Supplementary Fig. 6C). These lesions expanded, and at 6 months, $>50 \%$ of the ovarian surface was covered by malignant, Tomato+ cells, whereas at 9 months, the surface was almost entirely overrun (Supplementary Fig. 6C-F). Histologic examination again showed multi-villus neoplasia (Supplementary Fig. 6D and G), and IHC revealed highly proliferative tumors expressing HGSOC markers, including Wilms' tumor 1 (WT1) and Stathmin1 (Supplementary Fig. 6G). Neither hyperproliferation nor neoplasia was evident in the FT of LPT mice (Supplementary Fig. S7A), and the OSE-derived tumors also were PAX8-negative (Supplementary Fig. S7B).

\section{OSE-derived organoids also give rise to ovarian cancer}

We were unable to culture OSE organoids in the FTE medium. However, with additional factors, such as hydrocortisone, estrogen, LIF and forskolin, organoids could be propagated from single OSE cells (Fig. 5A). These organoids expressed epithelial markers, such as E-cadherin, but unlike FTE organoids, they did not express PAX8 (Fig. 5B). As noted above, Lgr5+ cells can repopulate the entire OSE, suggesting that they have stem/progenitor activity. Consistent with this notion, GFP ${ }^{\text {hi }}$ (Lgr5-expressing) cells accounted for almost all organoid-forming capability, compared with $\mathrm{GFP}^{\text {neg }}$ (Lgr5-negative) cells ( $\mathrm{P}<0.001$, Fig. 5C). We next generated OSE organoids from wild type, LP, LT and LPT mice, and added 4-OHT to the medium for $48 \mathrm{~h}$ to activate CRE. Following 4-OHT treatment, T121 was expressed in LT and LPT organoids, as expected (Fig. 5D). Organoid size was not affected, but mutant organoids were more condensed, containing larger numbers of cells (Fig. 5E). Although organoidforming capacity was similar among the 4 groups, LPT $(\mathrm{P}<0.001)$, and to a much lower extent $(\mathrm{P}<0.01), \mathrm{LT}$ organoids grew faster (Fig. 5G). Finally, we implanted all four types of organoids into the MFP and orthotopically (Fig. 5H). Consistent with the cognate GEMMs (Fig.4F), only LPT organoids induced metastasize tumors, and organoids and the tumors they evoked had similar histology and marker expression (Fig. 5I and J).

\section{Transcriptome analysis reveals similarities and differences between FTE- and OSE-derived HGSOC}

To molecularly characterize our HGSOC mouse models in an unbiased manner, we performed RNAseq on FTE-derived tumors (T-FT hereafter) from PTPT mice and OSE-derived tumors (T-O hereafter) from LPT mice. Pooled normal OSE (N-O hereafter) and FTE (N-FT hereafter) samples (3 each) were profiled as controls. Unsupervised analysis showed that FTE-derived (tumor and normal) samples segregated from OSE-derived (tumor and normal) samples (Fig. 6A). Multiple genes were differentially expressed in the two normal cell types (N-O vs $\mathrm{N}-\mathrm{FT}$ ) and in the tumors that derive from them (T-O vs T-FT); indeed, nearly 3,000 lineage-specific genes contributed to significant differences in gene expression (FDR-adjusted $\mathrm{P} \leq 0.05$ ) between FTE-and OSE-derived tumors, respectively (intersection between the N-O vs. N-FT and T-O vs. T-FT ovals in the Venn diagram in Fig. 6B; also see Supplementary Table S1A). Furthermore, supervised analysis revealed that the top 50 and top 100 differentially expressed genes between FTE-derived and OSE-derived tumors largely reflected differential gene expression in N-FT and N-O, although other (non-lineage) genes contribute to the top 1,000 DEGs (Supplementary Fig. S8). Comparing N-FTE and N-O, Pax8 $\left(\operatorname{logFC}=4.05, \mathrm{P}_{\text {adj }}=5.5{\mathrm{X} 10^{-14}}^{-1}, \operatorname{Ltf}\left(\operatorname{logFC}=-5.92, \mathrm{P}_{\mathrm{adj}}<1 \mathrm{X} 10^{-15}\right), \operatorname{Slc} 34 a 2\right.$ 
$\left(\operatorname{logFC}=4.84, \mathrm{P}_{\mathrm{adj}}<1 \mathrm{X} 10^{-15}\right)$, which are known FT-specific genes (53), are highly expressed both in N-FT and T-FT, compared with N-O and T-O. By contrast, the known OSE-specific gene $\operatorname{Lgr} 5\left(\operatorname{logFC}=5.54, \mathrm{P}_{\mathrm{adj}}=0\right)$ was expressed at significantly higher levels in N-O and T-O than N-FT and T-FT, as were Nr5a1 (encoding a transcriptional activator involved in sex determination and differentiation of steroidogenic tissues, $\operatorname{logFC}=6.5, \mathrm{P}_{\mathrm{adj}}<1 \mathrm{X} 10^{-}$ ${ }^{15}$ ), Gata4 (encoding a zinc finger TF), $L h x 9$ (encoding a target of GATA4, $\operatorname{logFC}=6.58, \mathrm{P}_{\text {adj }}<1 \times 10^{-15}$ ), and Unc45b (encoding a GATA4 chaperone, $\operatorname{logFC}=6.6, \mathrm{P}_{\mathrm{adj}}<1 \times 10^{-15}$ ); see Supplementary Fig. $\mathrm{S} 8$ and Supplementary Table 1B).

Next we searched for common DEGs in each sample type. Nearly 7,000 (6844) DEGs were seen in OSEderived tumors compared with normal OSE, whereas 5305 DEGs were yielded in T-FT vs N-FT (all at the $\mathrm{P}<0.05$ level). Of these, 1844 genes were shared between T-FT and T-O, compared with the cognate normal samples (Supplementary Fig. S8 and Supplementary Table S1C). KEGG analysis showed that DEGs in normal FTE, compared with normal OSE, were enriched for different pathways; e.g., peroxisome-related transcripts were enriched in the FTE, whereas ribosome-related genes, were enriched in the OSE. Although a substantial fraction of differential gene expression in T-FT vs T-O reflected lineage-specific gene expressions, tumors also shared multiple DEGs (compared with their cognate normal cells-of-origin). These were enriched for genes involved in pathways annotated as controlling the cell cycle, DNA replication and DNA repair (Fig.6C). Of note, the "p53 signaling pathway" was more enriched in the FTE-derived tumors, whereas homologous recombination, mismatch repair and nucleotide excision repair were more enriched in OSE-derived tumors (Fig. 6C). Similar to the KEGG analysis, the most enriched GO categories in all of the tumor sets involved cell/nuclear division and cell cycle control and DNA replication and repair, while none of the pathways enriched in the normal FT and normal OSE (Supplementary Fig. S9A). GSEA showed a predominance of G2/M checkpoint control and E2F target genes in all of the tumor samples (Fig. 6D and Supplementary Fig. S9B), the latter consistent with dysregulation of RB family/E2F regulation.

To assess the potential relevance of these mouse model findings with human genomic data, we compared protein-coding genes with known human orthologs (17, 598 genes) in each group (T-FT, T-O, N-FT, N-O), and selected genes that were uniquely expressed in each to generate group signatures (Supplementary Table S2). Comparison of the mean of scaled expression of the top 50, 100, 250 or 500 T-FT and T-O genes, respectively, with the other four groups showed the expected segregation of samples (Supplementary Fig. S10). We then applied the mouse T-FT and T-O scores (calculated for either the top 50, 100, 250 or 500 DEGs) to TCGA ovarian cancer data. This analysis revealed that human tumors classified as mesenchymal (mean enrichment score $=0.27,0.26$ and 0.25 with gene cutoff at Top 100, 250 and 500, respectively. $\mathrm{P}<0.001$ in all cutoffs when compared to other subtypes), and to a lesser extent, as immunoreactive, were more similar to the mouse T-FT, than to the mouse T-O, signature. By contrast, a larger proportion of proliferative type human tumors more closely resembled the mouse T-O signature (mean enrichment score $=0.13,0.16$ and 0.15 , respectively. $\mathrm{P}<0.05$ in all cutoffs when compared to other subtypes), whereas differentiated tumors segregated fairly equally into those more similar to FTE-derived (mean enrichment score $=-0.05,-0.04$ and -0.07 with gene cutoff at Top 100, 250 and 500, respectively) vs. OSE-derived mouse tumors (mean enrichment score $=-0.1,-0.13$ and -0.12 with gene cutoff at Top 100, 250 and 500, respectively), respectively (Fig. 6E and Supplementary Table S3). The robustness of the outcome independent of the number of T-FT and T$\mathrm{O}$ genes chosen gives us confidence in the reliability of this analysis. Furthermore, similar results were obtained by analyzing a distinct set of tumor transcriptomes [(22), data not shown)].

\section{The cell-of-origin can affect response to chemotherapeutic agents}


To ask if the cell-of-origin of HGSOC might affect therapeutic response, we tested the response of FTEand OSE-derived tumorigenic organoids to commonly used ovarian cancer drugs. PTPT (FTE) and LPT (OSE) organoids at day 4 of culture ( $>3$ passages post-Dox and 4-OHT treatment) were released from Matrigel, $\sim 150$ organoids were re-seeded in each well of 96-well plates pre-coated with Matrigel, and morphology and viability were assessed 5 days later. Tumorigenic FTE and OSE organoids responded similarly to niriparib, olaparib and gemcitabine (Fig. 7A-C). By contrast, FTE-derived (PTPT) organoids were significantly more sensitive that OSE (LPT) organoids to carboplatin $(\mathrm{P}<0.05$ at $10 \mu \mathrm{M})$ and, even more prominently, to paclitaxel $(\mathrm{P}<0.001$ at $5 \mu \mathrm{M}, 10$ $\mu \mathrm{M}$ and $25 \mu \mathrm{M}$ ) treatment (Fig. 7D and E). These data indicate that the cell-of-origin could influence response to at least some types of chemotherapy.

\section{DISCUSSION}

HGSOC is usually diagnosed late, as bulky multifocal disease that has metastasized widely to the peritoneal cavity, a presentation that makes identifying the cell-of-origin difficult (54). Although precursor lesions on the FT fimbriae have been reported in up to $60 \%$ of HGSOC cases (13-15), some studies are more consistent with an ovarian or peritoneal origin (5-7). Previous mouse modeling studies showed clearly that FTE can act as the cell-oforigin of HGSOC, but a similar role for OSE has not been excluded. Here, by introducing the same genetic abnormalities into FTE or OSE in GEMMs and organoid models, we have found that HGSOC can originate from either cell type (Supplementary Fig. S11). Importantly, FTE- and OSE-derived tumors differ in their metastatic behavior, transcriptomes and chemosensitivity.

Similar to a previous report, in which combined deletion of Tp53, Brca1/2, and Pten was driven by Pax8rtTA;TetOCre (37), PTPT mice rapidly developed STIC-like lesions and early ( $<2$ month) metastasis to the ovarian surface. Lineage tracing confirmed that Pax8 rtTA is expressed in FTE secretory cells, but importantly, consistent with a recent study (43), we find that Pax8-ciliated cells also derive from Pax $8+$ progenitors (Supplementary Fig. 2). Thus, while it is clear that HGSOC can arise from mouse FTE, we cannot be certain that FTE secretory cells (as opposed to Pax8-derived, but Pax 8- ciliated cells or a specialized Pax 8+ progenitor) are the actual/unique cell-of-origin in the FT. Others have found that the Ovgp1 promoter also can drive HGSOC $(38,39)$. This Cre line purportedly is secretory cell-specific (55), but lineage tracing studies have not been reported. In any event, there should now be little debate that a majority of HGSOC originates from FTE.

Unlike earlier studies using Pax8rtTA;TetOCre to drive combined Brca1/2;Pten;Tp53 deletion, which gave rise to STIC, invasive HGSOC, and eventually, peritoneal carcinomatosis, PTPT mice died prematurely from massive thymic hyperplasia. Subsequent analysis revealed unexpected, leaky expression of the TetOCre promoter in thymic epithelial cells. Previous work showed that E2F activation, resulting from RB family inactivation, leads to increased Foxn1 expression, which drives thymic epithelial cell proliferation (44). Presumably, Brca1/2;Pten;Tp53 deletion does not cause such hyperproliferation, although conceivably, these defects could affect other thymic epithelial cell properties and, indirectly, immune function, in such mice. Regardless, our results argue for caution in using the TetOCre line to study FTE biology and oncogenesis.

Although HGSOC usually arises from FTE, whether OSE can also give rise to HGSOC had been less clear. Studies in which oncogenic defects were induced by Ad-Cre injections into the ovarian bursa were interpreted as indicating an OSE cell-of-origin (56,57), although others have argued that such injections can result in infection of adjacent tissue, including the oviduct or even the uterus (58). Indeed, our lineage tracing experiments show clearly 
that bursal injection can target FTE as well as OSE. Nevertheless, several lines of evidence prove that mouse OSE can serve as the cell-of-origin for HGSOC, at least HGSOC induced by combined Tp53 mutation/inactivation and RB family inactivation. First, Ad-Cre-injected $T p 53^{R 172 H / f l} ; T 121$ mutant mice develop frank ovarian carcinoma even after salpingectomy. Moreover, $\mathrm{Lgr} 5$-Cre, which we confirm by lineage tracing marks OSE, but not FTE, in adult mice, also can drive HGSOC generation by combined RB family inactivation/Tp53 mutation/hemizygosity. Furthermore, OSE organoids in which the above genetic defects are induced in vitro, give rise to HGSOC-like lesions.

Although metastasis could not be evaluated in PTPT mice, organoid transplantation clearly shows that mouse FTE-derived HGSOC metastasizes widely. By establishing conditions for OSE-derived organoids, we could directly compare tumorigenesis by FTE- and OSE-derived HGSOC. Interestingly, these experiments suggest that the cell-of-origin can influence HGSOC behavior: FTE-derived tumors showed greater ability to disseminate, whereas OSE-derived HGSOC more commonly formed large, solitary lesions that show less frequent (although nonetheless significant) dissemination. Notably, human HGSOC also differs in its pattern of growth and metastasis, with some cases forming large primary tumors and fewer, less diffuse tumor deposits, whereas others show peritoneal carcinomatosis (59). Defined combinations of genetic defects (e.g., as found in TCGA) can be engineered into our FTE and OSE organoids, allowing various aspects of the transformation process (proliferation, polarity, apoptosis, necrosis, invasion) to be deconstructed in vitro and tumorgenicity to be assessed in vivo. It will be interesting to determine the extent to which the differential behavior observed here reflects the particular combination of cell-of-origin/oncogenic defects vs. effects of the cell-of-origin per se. Our RNAseq data, however, suggest that whereas common pathways and processes are de-regulated by imposing the same oncogenic insults on FTE and OSE, there are marked differences in which specific genes within these pathways/process are affected in the two cell types. Such differences might manifest as differential sensitivities to the two agents used as front line therapy for HGSOC, carboplatin and paclitaxel. In this regard, it is interesting to note that whereas repair genes are differentially expressed in both FTE- and OSE-derived tumors (compared with their cognate normal tissues), the extent of altered expression is greater in the latter. By contrast, signaling from p53-dependent pathways (presumably by alternative mechanisms, given the combined TP53 mutation/deletion in both types of tumors) is elevated to a greater extent in FTE-derived, than in OSE-derived tumors. Either or both of these differences might help explain the observed cell-of-origin-dependent differences in chemosensitivities.

Several lines of evidence suggest that our results are relevant for human HGSOC. First, a recent case report documented the development of stage IV HGSOC three years post- salpingectomy without evident STIC in the excised FT (60). Furthermore, recent exome sequencing (24), transcriptome (22) and ChiPseq experiments (23) support the notion that HGSOC can arise at sites other than the FTE. Our comparison of the transcriptome of FTEand OSE-derived HGSOC, respectively, compared with TCGA samples, indicates greater resemblance of some human HGSOCs, particularly proliferative type HGSOC, to OSE-derived tumors. Understanding the contribution of the cell-of-origin to HGSOC generation could be of substantial importance, given that our results indicate that tumorigenic OSE- and FTE-derived organoids bearing the same oncogenic defects have different biologic behavior, including sensitivities to anti-neoplastic drugs. 


\section{METHODS}

\section{Mice}

Rosa26-tdTomato [B6;129S6-Gt(ROSA)26SOR $\left.{ }^{\text {tm9(CAG-tdTomato)Hze }], ~[B 6 ; 129-G t(R O S A) 26 S o r T ~}{ }^{\text {MIsor }}\right]$, Lgr5-

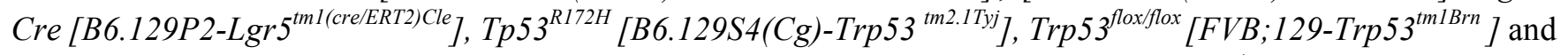
$n u / n u[N U / J]$ mice were obtained from the Jackson Laboratory. Conditional $\operatorname{TgK} 18 G T_{121}{ }^{t g /+}$ BAC transgenic mice (T121 mice) were described previously (36). Pax8rtTA and TetOcre strains were described previously (37). $T p 53^{R 172 H}$, Trp53 $3^{\text {floxflox }}$, T121 mice were interbred with Pax8rtTA and TetOcre mice to obtain Pax8rtTA;TetOcre;Tp53 ${ }^{\text {R172Hfl }}$ (PTP), Pax8rtTA;TetOcre;T121 (PTT) and Pax8rtTA; TetOcre; Tp53 ${ }^{\text {R172H/fl }}$;T121 (PTPT) mice, respectively. Trp53 ${ }^{R 172 H}, \operatorname{Trp} 53^{\text {floxflox }}, T 121$ and Lgr5Cre ${ }^{E R T 2}$ were interbred to obtain Lgr5Cre;Tp53 ${ }_{R 172 H / f l}$ (LP), Lgr5Cre;T121(LT), Lgr5Cre; Trp53 ${ }^{R 172 H / f l}$;T121 (LPT) mice, respectively. Rosa26-lacz and Rosa26tdTomato mice were bred to Pax8rtTA and TetOcre strains to obtain Pax8rtTA;TetOcre;Rosa26-LacZ and Pax8rtTA;TetOcre;Rosa26-tdTomato mice, respectively. When indicated, mice were euthanized by $\mathrm{CO}_{2}$ inhalation and FT and/or ovaries were harvested for histology and organoid culture. All animal experiments were approved by, and conducted in accordance with the procedures of, the IACUC at New York University School of Medicine (protocol no.170602).

\section{Animal experiments}

For lineage tracing of Pax8+FTE cells, Pax8rtTA; TetOcre;Rosa26-LacZ female mice (6-8 week old) were induced with $2 \mathrm{mg} / \mathrm{ml}$ doxycycline in their drinking water for 2 days. Mice were sacrificed, and their reproductive systems were collected, at 2 or 60 days after Dox administration. Lgr5-Cre;Rosa26-tdTomato mice (6-8 weeks old) were injected intraperitoneally with a single dose of 4-OHT in sesame oil $(10 \mathrm{mg} / \mathrm{ml})$ at a concentration of $2 \mathrm{mg}$ (in $200 \mu \mathrm{l}$ ), and then sacrificed at $48 \mathrm{~h}$ or 4 months after induction. Superovulation was performed by injection with 5 IU pregnant mare serum gonadotropin (PMSG, Sigma) and 5IU of human chorionic gonadotropin (hCG, Sigma), spaced $48 \mathrm{~h}$ apart. For Cre induction in PTP, PTT and PTPT mice, Dox $(2 \mathrm{mg} / \mathrm{ml})$ was added for 2 weeks to the drinking water of 6 week-old females, and relevant tissues were collected for H\&E and IHC after 1 month. For Cre induction in LP, LT and LPT mice, 6 week-old females were injected with 4-OHT, and mice were sacrificed at indicated time.

For ovarian bursa injection, recombinant adenovirus Ad5-CMV-Cre (Ad-Cre) was purchased from the Vector Development Lab at Baylor College of Medicine. Superovulation was performed in 6-8 week old female $T p 53^{R 172 H / f l} ; T 121$ mice, as described (35). Approximately 1.5 days later, a single injection of $10 \mu \mathrm{Ad}$-Cre $\left(10^{11}\right.$ $10^{12}$ infectious particles $/ \mathrm{ml}$ ) was delivered into one surgically exposed ovarian bursa, with the contralateral ovary serving as a control. Rosa26-LacZ female mice were used to test Cre expression and were euthanized 2 weeks postinjection. Salpingectomies on $T p 53^{R 172 H f f l} ; T 121$ females were performed 3 days after Ad-Cre injection, and mice were euthanized 3 months afterwards. Animals were routinely monitored for signs of distress, poor body condition, and tumor burden, and were euthanized according to veterinary recommendations. For survival experiments, the mice were monitored until their death or upon veterinary recommendation.

Organoids were amplified in 6-well plates coated with $200 \mu \mathrm{l}$ of Matrigel/well and supplemented with $2 \mathrm{ml}$ organoid growth medium. Orthotopic injections were performed on 8 week-old female mice. For surgeries, mice were anesthetized by intraperitoneal injection of $0.2 \mathrm{ml}$ xylazine hydrochloride, shaved, and cleaned with betadine. A midline dorsal incision was made, followed by an incision into the peritoneal cavity above the fat pad of the right ovary. The ovary was externalized through the incision and $0.5 \times 10^{6}$ cells/matrix mixture $(1: 1,15-20 \mu l)$ was injected by inserting a $27 \mathrm{G}$ needle into the fat pad. Only one ovary in each animal was injected, with the contralateral ovary serving as a control. The injected tissue was returned to the peritoneal cavity, the inner incision was sutured, 
and the outer incision was sealed with wound clips. For mammary fat pad injections, cells were resuspended with Matrigel $(1: 1$, around $15 \mu \mathrm{l})$ and injected into the mammary fat pad just inferior to the nipple of female mice (6-8 weeks) with $28 \mathrm{G}$ needle (BD insulin syringe). Mice that developed tumors were euthanized at the indicated times, when tumors ulcerated or reached a maximum diameter of $20 \mathrm{~mm}$, or when mice showed any sign of discomfort due to tumor burden.

\section{Organoid Cultures and Assays}

For FTE organoids, FT fimbriae from wild type, PTP, PTT and PTPT were dissected under a microscope, minced and digested with Collagenase type I and dispase $0.012 \%(\mathrm{w} / \mathrm{v})$ (STEMCELL Technologies) at $37^{\circ} \mathrm{C}$ for 1 hour, followed by incubation in TrypLE ${ }^{\mathrm{TM}}$ Express Enzyme (Thermo Fisher Scientific) for $10 \mathrm{~min}$ at $37^{\circ} \mathrm{C}$, and inactivation with FBS (Gibco) 1\% in DMEM media (Gibco). Dispersed FTE cells were passed through a strainer $(70 \mu \mathrm{m})$, mixed with Matrigel (BD Bioscience), seeded and cultured as described(61). After Matrigel solidified (10 min at $37^{\circ} \mathrm{C}$ incubator), culture medium was added. The medium was based on Ad+++ medium (AdDMEM/F12 (Invitrogen) with HEPES (Thermo Fisher Scientific), penicillin/streptomycin (Life Technologies), Glutamax (Life Technologies)), supplemented with B27 (Invitrogen), N2 supplement (Thermo Fisher Scientific), $1.25 \mathrm{mM} \mathrm{N}-$ acetylcysteine (Sigma), 50 ng/ml EGF (Thermo Fisher Scientific), 500 ng/ml RSPO1 (Peprotech) or R-spondin-1conditioned medium (25\%, v/v), and $100 \mathrm{ng} / \mathrm{ml}$ Noggin (Peprotech). For the first 3 days after thawing, culture medium was supplemented with $10 \mathrm{mM} \mathrm{Y-27632} \mathrm{(Sigma-Aldrich).} \mathrm{For} \mathrm{OSE} \mathrm{organoid} \mathrm{culture,} \mathrm{fat} \mathrm{and} \mathrm{adjacent}$ fallopian tubes were carefully removed under a microscope, and the ovaries were digested with $0.25 \%$ trypsin/EDTA (Invitrogen) and inactivated with DMEM media containing 1\% FBS (Gibco) at $37^{\circ} \mathrm{C}$ for $30 \mathrm{~min}$. Supernatants, containing cells stripped from the OSE, were seeded in Matrigel, and cultured in Ad+++ medium, supplemented with B27, N-acetylcysteine (1 mM, Sigma), WNT3a-conditioned medium (50\% v/v), R-spondin-1conditioned medium (10\% v/v), Noggin (100 ng/ml, Peprotech), EGF $(0.0125 \mu \mathrm{g} / \mathrm{ml})$, nicotinamide $(10 \mathrm{mM}$, Sigma), A83-01 (0.5 $\mu \mathrm{M}$, Tocris Bioscience), hydrocortisone (0.5 $\mu \mathrm{g} / \mathrm{ml}$, Sigma), and $\beta$-estradiol (100 $\mathrm{nM}$, Sigma). Passaging and freezing were carried out as described above for FTE organoids. WNT3a- and R-spondin-1conditioned media were prepared as described (62).

Organoid medium was changed every 2-3 days, and they were passaged approximately 1:4 (10,000 cells/well) every 6-8 days. For passage, growth medium was removed, and the Matrigel was resuspended in cold Cultrex ${ }^{\circledR}$ Organoid Harvesting Solution and transferred to a 15-ml Falcon tube, which was placed on ice for 15 minutes. Organoids were recovered by centrifugation at $1,000 \mathrm{~g}$ for 5 minutes and resuspended in $500 \mu \mathrm{l}$ TrypLE Express Enzyme (Gibco) for 10 minutes at $37^{\circ} \mathrm{C}$. Cells were then seeded as indicated for each experiment. For freezing, cells were resuspended in organoid medium with 10\% DMSO and 10\% FBS, cooled, and stored in liquid nitrogen.

To activate Cre in vitro, $0.5 \mu \mathrm{l}$ of Dox $(1 \mathrm{mg} / \mathrm{ml})$ were added to $500 \mu \mathrm{l}$ of freshly isolated PTP, PTT and PTPT cells, which were plated as described above. Similarly, $1 \mu \mathrm{g} / \mathrm{ml} 4-\mathrm{OHT}$ was used to activate CRE in LP, LT and LPT organoids. All data were generated at least 3 passages after induction. Organoid size was quantified as the surface area of horizontal cross sections. If all organoids in a well could not be measured, several random, nonoverlapping pictures were acquired from each well using an Invitrogen ${ }^{\mathrm{TM}}$ EVOS ${ }^{\mathrm{TM}}$ FL Digital Inverted Fluorescence Microscope, and analyzed by using ImageJ software. Organoid perimeters for area measurements were defined manually and by automated determination using the "Analyze Particle" function of ImageJ, with investigator verification of the automated determinations. Organoids touching the edges of images were excluded from counting. 
To compare organoid-forming efficiency of different genotypes, 5,000 cells were seeded into a 24-well plate, total organoid number was counted under a light microscope after 5-7 days in culture, and the percentage of organoids formed relative to organoids formed by wild type cells was calculated.

For in vitro growth curves, organoids were incubated in TrypLE Express (Gibco) for $15 \mathrm{~min}$ at $37^{\circ} \mathrm{C}$, followed by an additional 5 min digestion in Dispase I. Isolated cells were passed through a strainer, seeded at 2 $\mathrm{x} 10^{4}$ cells/well in a 24 well plate and placed in culture medium. At the indicated times, cells were recovered as described above, and viable counts were obtained by trypan blue exclusion.

Drugs were tested in organoids based on a previously described protocol (62). Briefly, organoids in culture at day 4 were released from Matrigel and diluted to 50 organoids $/ \mu 1$ in growth medium lacking $\mathrm{N}$-acetylcysteine and Y-27632. Clear bottom 96-well plates were coated with $20 \mu 1$ Matrigel before the addition of $30 \mu 1$ of organoid suspension. The indicated concentrations of paclitaxel (Selleck Chem), carboplatin (Sigma), olaparib (Selleck Chem), niraparib (Selleck Chem), or DMSO (control) were added in triplicate. On day 5 of treatment, media were removed and the Matrigel drops were suspended in $40 \mu$ CellTiter-Glo 3D (Promega) and $80 \mu 1$ advanced AdDMEM/F12, incubated for $30 \mathrm{~min}$ at room temperature before luminescence was measured in FlexStation 3 Multi-Mode Microplate Reader. Results were normalized to DMSO controls.

Invasion was assessed by using chambers with $8 \mu \mathrm{m}$ pore size polycarbonate membrane (Transwell) inserts (Costar). Matrigel $(30 \mu \mathrm{l})$ was added to the chambers and allowed to solifiy at $37^{\circ} \mathrm{C}$ for 10 minutes. Wild type, PTP, PTT, or PTPT cells $\left(2 \times 10^{4} / 50 \mu \mathrm{l} \mathrm{Ad}+++\right.$ medium/well) were seeded into the Matrigel-coated top chamber, and allowed to attach for 12 hours, followed by the addition of $500 \mu \mathrm{l}$ of culture medium to each well. After an additional 96 hours of incubation, the upper surface of the Transwell membrane was scrubbed carefully several times with a cotton swab soaked in PBS to remove non-invaded cells. The lower membrane was then rinsed with PBS carefully several times, and cells that had invaded were visualized by staining with crystal violet and counted under a microscope. Invasion was calculated as the average number of cells per 10x field, determined by Image J software.

\section{FACS}

Ovaries from Lgr5-Cre ( $L g r 5-E G F P-i r e s-C r e^{E R T 2}$ ) females (6-8 weeks) were digested as described above and recovered OSE cells were passed through a strainer $(40 \mu \mathrm{m})$ to obtain single-cell suspensions. OSE cells were pelleted by centrifugation at 1,000 g for 5 min and resuspended in PBS containing 2\% FBS, Rock inhibitor (Y27632, $10 \mu \mathrm{M}$, STEMCELL Technologies Inc.), DAPI $(1 \mu \mathrm{g} / \mathrm{ml})$, FACS was performed immediate by using a $\mathrm{MoFlo}^{\mathrm{TM}} \mathrm{XDP}$, and sorted GFP ${ }^{\text {hi }}$ and $\mathrm{GFP}^{\text {neg }}$ cells were seeded at 5000/well. Organoids were counted 6 days later, and organoid forming efficiency was calculated as number of the organoids/number of cells seeded, pooled from three biological replicates.

\section{Histology and Immunostaining}

Tissues were fixed in $4 \%$ paraformaldehyde (PFA) in PBS at $4^{\circ} \mathrm{C}$ for 4 hours. Organoids were fixed in $4 \%$ PFA for 15 minutes and placed in Histogel (Thermo Fisher Scientific) before tissue processing and embedding. Formalin-fixed paraffin-embedded (FFPE) tissue sections $(5 \mu \mathrm{m})$ were de-paraffinized, rehydrated, and then stained with hematoxylin and eosin (H\&E) or subjected to IHC. For antigen retrieval, slides were autoclaved in $0.01 \mathrm{M}$ citrate buffer ( $\mathrm{pH}$ 6.0). Endogenous peroxidase activity was quenched in $3 \% \mathrm{H}_{2} \mathrm{O}_{2}$ in methanol for 15 min and sections were blocked with $0.5 \%$ BSA-PBS for $1 \mathrm{~h}$. Primary antibodies were added overnight at $4^{\circ} \mathrm{C}$, then washed with PBS 3x10 min, incubated with HRP-labeled secondary antibodies, and after washing with PBS 3x for 10 minutes each, signals were visualized by using the HRP Polymer Detection Kit and DAB peroxidase (HRP) 
substrate (34002, Life Technologies). Primary antibodies included: Ki67 1:200 (ab15580, Abcam), $\gamma$-H2AX 1:500 (05-636, Thermo Fisher Scientific), CK7 1:200 (ab181598, Abcam), Stathmin-1 1:200 (3352S, cell signaling), P16 1:200 (sc-1661, Santa Cruz), PAX8 1:200 (10336-1-AP, Proteintech), p53 1:800 (P53-CM5P-L, Leica). Secondary antibodies included: goat anti-chicken IgY-HRP 1:200 (sc-2428, Santa Cruz), goat anti-rabbit IgG-HRP 1:200 (sc2030, Santa Cruz).

Immunofluorescence was performed in frozen tissue sections $(5 \mu \mathrm{m})$ or in whole organoids released by gently dissolving the Matrigel in ice-cold PBS. Following fixation as above, cells were permeabilized in PBS containing $0.5 \%$ Triton X-100 and blocked in PBS containing 1\% BSA, 3\% normal goat serum and 0.2\% Triton X100. Primary antibodies were incubated at $4^{\circ} \mathrm{C}$ overnight, and sections were washed in PBS $3 \mathrm{x}$ for 10 minutes each, followed by incubation with DAPI $(2 \mu \mathrm{g} / \mathrm{ml})$ and Alexa 488-, 555- or 647-conjugated anti-chicken, anti-rabbit or anti-mouse antibodies, as indicated. After washing, samples were mounted with ProLong Gold antifade reagent (Life Technology). Primary antibodies were: GFP 1:300 (ab13970, Abcam), WT1 1:200 (ab15249, Abcam), Ecadherin 1:200 (ab15148, Abcam). Secondary antibodies included: goat anti-mouse IgG, Alexa Fluor ${ }^{\circledR} 647$ conjugate 1:200 (A28181, Thermo Fisher Scientific), goat anti-rabbit IgG, Alexa Fluor ${ }^{\circledR} 555$ conjugate 1:200 (A27039, Thermo Fisher Scientific), and goat anti-chicken IgY H\&L (Alexa Fluor ${ }^{\circledR} 488$ ) 1:200 (ab150169, Abcam).

\section{Laser capture microdissection and RNA extraction}

Tissues, including normal ovary and fallopian tube (fimbriae), ovarian tumors from LPT females, and STICs from PTPT mice, were embedded in FSC 22 Clear Frozen Section Compound and immediately frozen with liquid $\mathrm{N}_{2}$. The resultant blocks were cut at 5-8 $\mu \mathrm{m}$ and mounted on a PEN membrane frame (Leica), followed by air-drying the slides for 30 minutes at room temperature. Laser capture was performed with a Leica LMD6000 laser microdissection system, and excised pieces were harvested into $200 \mu 1$ RNase-free tubes, which were carefully recovered from the microscope, centrifuged, and placed on ice. RNA was extracted by using the miRNeasy mini Kit (Qiagen), following the manufacturer's instruction.

\section{RNA-Sequencing and Analysis}

Libraries were prepared using the Illumina TruSeq Stranded Total RNA Sample Preparation Kit. and sequenced on an Illumina HiSeq 4000 sequencer using 150bp paired-end reads by the Perlmutter Cancer Center Genome Technology Center shared resource (GTC). Sequencing results were demultiplexed and converted to FASTQ format using Illumina bcl2fastq software. The average number of read pairs was $60.3 \mathrm{M}$ per sample. Data were processed by the Perlmutter Cancer Center Applied Bioinformatics Laboratory shared resource (ABL). The reads were adapter and quality trimmed with Trimmomatic [http://www.ncbi.nlm.nih.gov/pubmed/24695404] and then aligned to the mouse genome (build mm10/GRCm38) using the splice-aware STAR aligner [http://www.ncbi.nlm.nih.gov/pubmed/23104886]. The featureCounts program [https://www.ncbi.nlm.nih.gov/pubmed/24227677] was utilized to generate counts for each gene based on how many aligned reads overlap its exons. These counts were then normalized and used to test for differential expression using negative binomial generalized linear models implemented by the DESeq2 $\mathrm{R}$ package [http://www.ncbi.nlm.nih.gov/pubmed/25516281]. Statistical analysis and visualization of gene sets were performed using the clusterProfiler R package [https://www.ncbi.nlm.nih.gov/pubmed/22455463].

FT/O-specific gene signatures were determined by calculating differentially upregulated genes pair-wise between all four examined groups. For each group, they were ranked by the highest p-value across all three comparisons for each gene. The genes were subset to protein coding and with available human ortholog predictions in any of the 14 databases indexed by the HUGO Gene Nomenclature Committee Comparison of Orthology 
Predictions resource. To measure the FT/O enrichment in individual samples, the normalized counts were scaled and centered, and the mean was calculated for all genes comprising the signature. TCGA Ovarian Cancer batch effects normalized mRNA data and molecular subtypes were retrieved from the UCSC Xena Pan-Cancer Atlas hub.

\section{Quantification and statistical analysis}

Unless otherwise specified, data are presented as mean \pm SEM. Survival rates were analyzed by log-rank test, using GraphPad Prism software. P values were determined by two-tailed Student's t-test, unless otherwise specified, with $\mathrm{P}<0.05$ considered statistically significant.

\section{Acknowledgments}

We thank Terry Van Dyke for sharing the T121 mice. We would also like to acknowledge technical support for this work provided by the PCC Experimental Pathology, Microscopy, GTC and ABL shared resources (P30CA016087). We also thank Drs. Jiyuan Hu (Division of Biostatistics), Chan Wang (Division of Biostatistics), Kwan Ho Tang (PCC) and Victor Ho (Princess Margaret Cancer Center) for advice and discussion. Initial work on this project was supported by grant-MOP-191992 from the Canadian Institutes for Health Research to B.G.N. D.A.L is supported by the Department of Defense Ovarian Cancer Research Program (W81XWH-15-1-0429), and S. Z. was supported by a post-doctoral fellowship from the Ovarian Cancer Research Fund Alliance. 


\section{REFERENCE}

1. Kobel M, Kalloger SE, Huntsman DG, Santos JL, Swenerton KD, Seidman JD, et al. Differences in tumor type in low-stage versus high-stage ovarian carcinomas. Int J Gynecol Pathol 2010;29(3):203-11 doi 10.1097/PGP.0b013e3181c042b6.

2. Klotz DM, Wimberger P. Cells of origin of ovarian cancer: ovarian surface epithelium or fallopian tube? Arch Gynecol Obstet 2017;296(6):1055-62 doi 10.1007/s00404-017-4529-z.

3. Kurman RJ. Origin and molecular pathogenesis of ovarian high-grade serous carcinoma. Ann Oncol 2013;24 Suppl 10:x16-21 doi 10.1093/annonc/mdt463.

4. Karnezis AN, Cho KR, Gilks CB, Pearce CL, Huntsman DG. The disparate origins of ovarian cancers: pathogenesis and prevention strategies. Nat Rev Cancer 2017;17(1):65-74 doi 10.1038/nrc.2016.113.

5. Auersperg N. Ovarian surface epithelium as a source of ovarian cancers: unwarranted speculation or evidence-based hypothesis? Gynecol Oncol 2013;130(1):246-51 doi 10.1016/j.ygyno.2013.03.021.

6. Auersperg N, Woo MM, Gilks CB. The origin of ovarian carcinomas: a developmental view. Gynecol Oncol 2008; 110(3):452-4 doi 10.1016/j.ygyno.2008.05.031.

7. Bell DA, Scully RE. Early de novo ovarian carcinoma. A study of fourteen cases. Cancer 1994;73(7):185964.

8. Scully RE. Pathology of ovarian cancer precursors. J Cell Biochem Suppl 1995;23:208-18.

9. Pothuri B, Leitao MM, Levine DA, Viale A, Olshen AB, Arroyo C, et al. Genetic analysis of the early natural history of epithelial ovarian carcinoma. PLoS One 2010;5(4):e10358 doi 10.1371/journal.pone.0010358.

10. Zweemer RP, van Diest PJ, Verheijen RH, Ryan A, Gille JJ, Sijmons RH, et al. Molecular evidence linking primary cancer of the fallopian tube to BRCA1 germline mutations. Gynecol Oncol 2000;76(1):45-50 doi 10.1006/gyno.1999.5623.

11. Colgan TJ, Murphy J, Cole DE, Narod S, Rosen B. Occult carcinoma in prophylactic oophorectomy specimens: prevalence and association with BRCA germline mutation status. Am J Surg Pathol 2001;25(10):1283-9.

12. Rebbeck TR, Lynch HT, Neuhausen SL, Narod SA, Van't Veer L, Garber JE, et al. Prophylactic oophorectomy in carriers of BRCA1 or BRCA2 mutations. N Engl J Med 2002;346(21):1616-22 doi 10.1056/NEJMoa012158.

13. Labidi-Galy SI, Papp E, Hallberg D, Niknafs N, Adleff V, Noe M, et al. High grade serous ovarian carcinomas originate in the fallopian tube. Nat Commun 2017;8(1):1093 doi 10.1038/s41467-017-009621.

14. Bowtell DD, Bohm S, Ahmed AA, Aspuria PJ, Bast RC, Jr., Beral V, et al. Rethinking ovarian cancer II: reducing mortality from high-grade serous ovarian cancer. Nat Rev Cancer 2015;15(11):668-79 doi $10.1038 / \mathrm{nrc} 4019$.

15. Kindelberger DW, Lee Y, Miron A, Hirsch MS, Feltmate C, Medeiros F, et al. Intraepithelial carcinoma of the fimbria and pelvic serous carcinoma: Evidence for a causal relationship. Am J Surg Pathol 2007;31(2):161-9 doi 10.1097/01.pas.0000213335.40358.47.

16. Harmsen MG, IntHout $\mathrm{J}$, Arts-de Jong $\mathrm{M}$, Hoogerbrugge $\mathrm{N}$, Massuger LF, Hermens RP, et al. Salpingectomy With Delayed Oophorectomy in BRCA1/2 Mutation Carriers: Estimating Ovarian Cancer Risk. Obstet Gynecol 2016;127(6):1054-63 doi 10.1097/AOG.0000000000001448.

17. Kuhn E, Kurman RJ, Vang R, Sehdev AS, Han G, Soslow R, et al. TP53 mutations in serous tubal intraepithelial carcinoma and concurrent pelvic high-grade serous carcinoma--evidence supporting the clonal relationship of the two lesions. J Pathol 2012;226(3):421-6 doi 10.1002/path.3023.

18. Cancer Genome Atlas Research N. Integrated genomic analyses of ovarian carcinoma. Nature 2011;474(7353):609-15 doi 10.1038/nature10166.

19. Mehra K, Mehrad M, Ning G, Drapkin R, McKeon FD, Xian W, et al. STICS, SCOUTs and p53 signatures; a new language for pelvic serous carcinogenesis. Front Biosci (Elite Ed) 2011;3:625-34. 
20. Gilks CB, Irving J, Kobel M, Lee C, Singh N, Wilkinson N, et al. Incidental nonuterine high-grade serous carcinomas arise in the fallopian tube in most cases: further evidence for the tubal origin of high-grade serous carcinomas. Am J Surg Pathol 2015;39(3):357-64 doi 10.1097/PAS.0000000000000353.

21. Bashashati A, Ha G, Tone A, Ding J, Prentice LM, Roth A, et al. Distinct evolutionary trajectories of primary high-grade serous ovarian cancers revealed through spatial mutational profiling. J Pathol 2013;231(1):21-34 doi 10.1002/path.4230.

22. Ducie J, Dao F, Considine M, Olvera N, Shaw PA, Kurman RJ, et al. Molecular analysis of high-grade serous ovarian carcinoma with and without associated serous tubal intra-epithelial carcinoma. Nat Commun 2017;8(1):990 doi 10.1038/s41467-017-01217-9.

23. Kate Lawrenson MASF, Felipe Segato, Janet M. Lee, Rosario I. Corona, Ji-Heui, Seo SC, Yvonne G. Lin, Tanja Pejovic, Paulette Mhawech-Fauceglia, Ronny Drapkin, Beth Y. Karlan, Dennis J. Hazelett, Matthew L. Freedman, Simon A. Gayther, Houtan Noushmhr. Integrated Molecular Profiling Studies to Characterize the Cellular Origins of High-Grade Serous Ovarian Cancer. bioRxiv 2018:1-22 doi doi: http://dx.doi.org/10.1101/330597.

24. Eckert MA, Pan S, Hernandez KM, Loth RM, Andrade J, Volchenboum SL, et al. Genomics of Ovarian Cancer Progression Reveals Diverse Metastatic Trajectories Including Intraepithelial Metastasis to the Fallopian Tube. Cancer Discov 2016;6(12):1342-51 doi 10.1158/2159-8290.CD-16-0607.

25. McDaniel AS, Stall JN, Hovelson DH, Cani AK, Liu CJ, Tomlins SA, et al. Next-Generation Sequencing of Tubal Intraepithelial Carcinomas. JAMA Oncol 2015;1(8):1128-32 doi 10.1001/jamaoncol.2015.1618.

26. Kommoss F, Faruqi A, Gilks CB, Lamshang Leen S, Singh N, Wilkinson N, et al. Uterine Serous Carcinomas Frequently Metastasize to the Fallopian Tube and Can Mimic Serous Tubal Intraepithelial Carcinoma. Am J Surg Pathol 2017;41(2):161-70 doi 10.1097/PAS.0000000000000757.

27. Rabban JT, Vohra P, Zaloudek CJ. Nongynecologic metastases to fallopian tube mucosa: a potential mimic of tubal high-grade serous carcinoma and benign tubal mucinous metaplasia or nonmucinous hyperplasia. Am J Surg Pathol 2015;39(1):35-51 doi 10.1097/PAS.0000000000000293.

28. Reyes C, Murali R, Park KJ. Secondary Involvement of the Adnexa and Uterine Corpus by Carcinomas of the Uterine Cervix: A Detailed Morphologic Description. Int J Gynecol Pathol 2015;34(6):551-63 doi 10.1097/PGP.0000000000000206.

29. Ng A, Barker N. Ovary and fimbrial stem cells: biology, niche and cancer origins. Nat Rev Mol Cell Biol 2015;16(10):625-38 doi 10.1038/nrm4056.

30. Auersperg N. The origin of ovarian carcinomas: a unifying hypothesis. Int J Gynecol Pathol 2011;30(1):1221 doi 10.1097/PGP.0b013e3181f45f3e.

31. Cheng W, Liu J, Yoshida H, Rosen D, Naora H. Lineage infidelity of epithelial ovarian cancers is controlled by HOX genes that specify regional identity in the reproductive tract. Nat Med 2005;11(5):531-7 doi $10.1038 / \mathrm{nm} 1230$.

32. Flesken-Nikitin A, Hwang CI, Cheng CY, Michurina TV, Enikolopov G, Nikitin AY. Ovarian surface epithelium at the junction area contains a cancer-prone stem cell niche. Nature 2013;495(7440):241-5 doi 10.1038/nature11979.

33. Xing D, Orsulic S. A mouse model for the molecular characterization of brca1-associated ovarian carcinoma. Cancer Res 2006;66(18):8949-53 doi 10.1158/0008-5472.CAN-06-1495.

34. Kinross KM, Montgomery KG, Kleinschmidt M, Waring P, Ivetac I, Tikoo A, et al. An activating Pik3ca mutation coupled with Pten loss is sufficient to initiate ovarian tumorigenesis in mice. J Clin Invest 2012;122(2):553-7 doi 10.1172/JCI59309.

35. Flesken-Nikitin A, Choi KC, Eng JP, Shmidt EN, Nikitin AY. Induction of carcinogenesis by concurrent inactivation of $\mathrm{p} 53$ and $\mathrm{Rb} 1$ in the mouse ovarian surface epithelium. Cancer Res 2003;63(13):3459-63.

36. Szabova L, Yin C, Bupp S, Guerin TM, Schlomer JJ, Householder DB, et al. Perturbation of Rb, p53, and Brca1 or Brca2 cooperate in inducing metastatic serous epithelial ovarian cancer. Cancer Res 2012;72(16):4141-53 doi 10.1158/0008-5472.CAN-11-3834.

37. Perets R, Wyant GA, Muto KW, Bijron JG, Poole BB, Chin KT, et al. Transformation of the fallopian tube secretory epithelium leads to high-grade serous ovarian cancer in Brca;Tp53;Pten models. Cancer Cell 2013;24(6):751-65 doi 10.1016/j.ccr.2013.10.013. 
38. Sherman-Baust CA, Kuhn E, Valle BL, Shih Ie M, Kurman RJ, Wang TL, et al. A genetically engineered ovarian cancer mouse model based on fallopian tube transformation mimics human high-grade serous carcinoma development. J Pathol 2014;233(3):228-37 doi 10.1002/path.4353.

39. Zhai Y, Wu R, Kuick R, Sessine MS, Schulman S, Green M, et al. High-grade serous carcinomas arise in the mouse oviduct via defects linked to the human disease. J Pathol 2017;243(1):16-25 doi 10.1002/path.4927.

40. Van Keymeulen A, Lee MY, Ousset M, Brohee S, Rorive S, Giraddi RR, et al. Reactivation of multipotency by oncogenic PIK3CA induces breast tumour heterogeneity. Nature 2015;525(7567):119-23 doi 10.1038/nature14665.

41. Turashvili G, Brogi E. Tumor Heterogeneity in Breast Cancer. Front Med (Lausanne) 2017;4:227 doi 10.3389/fmed.2017.00227.

42. Sutherland KD, Visvader JE. Cellular Mechanisms Underlying Intertumoral Heterogeneity. Trends Cancer 2015;1(1):15-23 doi 10.1016/j.trecan.2015.07.003.

43. Ghosh A, Syed SM, Tanwar PS. In vivo genetic cell lineage tracing reveals that oviductal secretory cells self-renew and give rise to ciliated cells. Development 2017;144(17):3031-41 doi 10.1242/dev.149989.

44. Garfin PM, Min D, Bryson JL, Serwold T, Edris B, Blackburn CC, et al. Inactivation of the RB family prevents thymus involution and promotes thymic function by direct control of Foxn1 expression. J Exp Med 2013;210(6):1087-97 doi 10.1084/jem.20121716.

45. Drost J, Clevers H. Organoids in cancer research. Nat Rev Cancer 2018 doi 10.1038/s41568-018-0007-6.

46. Dutta D, Heo I, Clevers H. Disease Modeling in Stem Cell-Derived 3D Organoid Systems. Trends Mol Med 2017;23(5):393-410 doi 10.1016/j.molmed.2017.02.007.

47. Kessler M, Hoffmann K, Brinkmann V, Thieck O, Jackisch S, Toelle B, et al. The Notch and Wnt pathways regulate stemness and differentiation in human fallopian tube organoids. Nat Commun 2015;6:8989 doi $10.1038 /$ ncomms 9989.

48. Laury AR, Hornick JL, Perets R, Krane JF, Corson J, Drapkin R, et al. PAX8 reliably distinguishes ovarian serous tumors from malignant mesothelioma. Am J Surg Pathol 2010;34(5):627-35 doi 10.1097/PAS.0b013e3181da7687.

49. Stewart JM, Shaw PA, Gedye C, Bernardini MQ, Neel BG, Ailles LE. Phenotypic heterogeneity and instability of human ovarian tumor-initiating cells. Proc Natl Acad Sci U S A 2011;108(16):6468-73 doi 10.1073/pnas.1005529108.

50. Cybulska P, Stewart JM, Sayad A, Virtanen C, Shaw PA, Clarke B, et al. A Genomically Characterized Collection of High-Grade Serous Ovarian Cancer Xenografts for Preclinical Testing. Am J Pathol 2018; 188(5):1120-31 doi 10.1016/j.ajpath.2018.01.019.

51. Auersperg N, Wong AS, Choi KC, Kang SK, Leung PC. Ovarian surface epithelium: biology, endocrinology, and pathology. Endocr Rev 2001;22(2):255-88 doi 10.1210/edrv.22.2.0422.

52. $\mathrm{Ng} \mathrm{A}$, Tan S, Singh G, Rizk P, Swathi Y, Tan TZ, et al. Lgr5 marks stem/progenitor cells in ovary and tubal epithelia. Nat Cell Biol 2014;16(8):745-57 doi 10.1038/ncb3000.

53. O'Shannessy DJ, Jackson SM, Twine NC, Hoffman BE, Dezso Z, Agoulnik SI, et al. Gene expression analyses support fallopian tube epithelium as the cell of origin of epithelial ovarian cancer. Int J Mol Sci 2013;14(7):13687-703 doi 10.3390/ijms140713687.

54. Swisher EM, Garcia RL, Kilgore MR, Norquist BM. Culprit or Bystander? The Role of the Fallopian Tube in "Ovarian" High-Grade Serous Carcinoma. Cancer Discov 2016;6(12):1309-11 doi 10.1158/21598290.CD-16-1197.

55. Wu R, Zhai Y, Kuick R, Karnezis AN, Garcia P, Naseem A, et al. Impact of oviductal versus ovarian epithelial cell of origin on ovarian endometrioid carcinoma phenotype in the mouse. J Pathol 2016;240(3):341-51 doi 10.1002/path.4783.

56. Bobbs AS, Cole JM, Cowden Dahl KD. Emerging and Evolving Ovarian Cancer Animal Models. Cancer Growth Metastasis 2015;8(Suppl 1):29-36 doi 10.4137/CGM.S21221.

57. Stuckelberger S, Drapkin R. Precious GEMMs: emergence of faithful models for ovarian cancer research. J Pathol 2018;245(2):129-31 doi 10.1002/path.5065. 
58. Sale S, Orsulic S. Models of ovarian cancer metastasis: Murine models. Drug Discov Today Dis Models 2006;3(2):149-54 doi 10.1016/j.ddmod.2006.05.006.

59. Lengyel E. Ovarian cancer development and metastasis. Am J Pathol 2010;177(3):1053-64 doi 10.2353/ajpath.2010.100105.

60. Sato E, Nakayama K, Ishikawa M, Nakamura K, Ishibashi T, Kyo S. High-grade serous ovarian cancer 3 years after bilateral salpingectomy: A case report. Mol Clin Oncol 2017;6(2):201-3 doi 10.3892/mco.2016.1105.

61. Sato T, Vries RG, Snippert HJ, van de Wetering M, Barker N, Stange DE, et al. Single Lgr5 stem cells build crypt-villus structures in vitro without a mesenchymal niche. Nature 2009;459(7244):262-5 doi 10.1038/nature07935.

62. Sachs N, de Ligt J, Kopper O, Gogola E, Bounova G, Weeber F, et al. A Living Biobank of Breast Cancer Organoids Captures Disease Heterogeneity. Cell 2018;172(1-2):373-86 e10 doi 10.1016/j.cell.2017.11.010. 


\section{FIGURE LEGENDS}

Figure 1. Combined Tp53 mutation/RB family inactivation in fallopian tube epithelium results in HGSOC. A, Schematic showing the Pax8rtTA, TetOCre, and Rosa26-lacZ alleles. B, Whole mount (Left panel) and 10Xmagnified histological section (right panel) of genital tracts of Pax8rtTA;TetOCre;Rosa26-LacZ female mice, treated with Dox $(2 \mathrm{mg} / \mathrm{ml}$ in drinking water for 2 weeks), stained with X-gal to detect $\beta$-galactosidase and counterstained with eosin. C, Schematic of Pax8rtTA;TetOcre; Tp53 $3^{R 172 H /} ; T 121$ (PTPT) mice. D, Immunofluorescence staining for GFP (marker for keratin 18+ FTE cells, green), Ki67 (proliferation marker, red) and DAPI (nuclear stain, blue) with or without Dox treatment as in B. Scale bar: $10 \mu \mathrm{m}$. E, Representative H\&Estained section of fallopian tube from PTPT mice with or without Dox $(2 \mathrm{mg} / \mathrm{ml}$ in drinking water for $1 \mathrm{month})$. Boxed regions in left panels are magnified in the right panel of each pair. Scale bar: $50 \mu \mathrm{m}$. F, \% mice of each genotype with STIC, assessed after 1 month of Dox treatment. G, Representative H\&E-stained ovary sections from PTPT mice, without or with Dox treatment for 1 month. Boxed regions in left panels are magnified in the right panel of each pair. The yellow dashed line shows the border between OSE and underlying cells. Arrows show areas of neoplasia on ovarian surface. Scale bar: $50 \mu \mathrm{m} . \mathbf{H}, \%$ of mice of each genotype that developed ovarian metastasis, assessed after 1 month of Dox treatment. I and J, IHC for key STIC markers in representative sections of FTE (top) and OSE (bottom) of PTPL mice. Scale bar: $10 \mu \mathrm{m}$.

Figure 2. Establishment and characterization of FTE organoids. A, Bright field image of organoid developing from single FTE cell at the indicated days of culture. Scale bar, $20 \mu \mathrm{m}$. B, Immunofluorescence staining for ciliated cell marker acetyl- $\alpha$-tubulin (green) and secretory cell marker PAX8 (red) in FTE organoid after 7 days in culture. Scale bar, $20 \mu \mathrm{m}$. C, Top: Schematic showing generation of FTE organoids from PTPT mice. Bottom: GFP in organoids with or without Dox treatment; Dox $(500 \mathrm{ng} / \mathrm{ml})$ was added after seeding cells and GFP was gone in PTPT organoids after 2 passages. Scale bar, $10 \mu \mathrm{m}$. D, Sections of PTPT organoids after 2 consecutive passages, with or without Dox treatment $(500 \mathrm{ng} / \mathrm{ml})$, stained with H\&E or subjected to immunofluorescence or IHC staining for the indicated markers. Scale bar, $20 \mu \mathrm{m}$. E, Organoids were established from the indicated mice, incubated with EdU $(2 \mu \mathrm{M})$ and DAPI $(1 \mu \mathrm{g} / \mathrm{ml})$ for 2 hours on day 7 of culture, and visualized by immunofluorescence. Scale bar, $20 \mu \mathrm{m}$. F, \% EdU-positive cells in organoids established from the indicated mice. G, Representative immunofluorescence images of PAX8 (red) and acetylated- $\alpha$-tubulin (green) staining of the indicated organoid lines at day 7 of culture. Scale bar, $20 \mu \mathrm{m}$. H, \% ciliated cells (aceylated- $\alpha$-tubulin + ) in organoids established from the indicated mice. I, Representative images of the bottom surface of Transwell units seeded with the indicated organoids. J, Quantification of invasive cells in I. Data represent means \pm SEM from three mice of each genetic background. $* \mathrm{P}<0.05,{ }^{*} \mathrm{P}<0.01,{ }^{* * *} \mathrm{P}<0.001$.

Figure 3. Transplanted organoids recapitulate HGSOC progression and metastasis. A, Scheme depicting organoid transplantation. B, \% of tumors formed in mammary fat pads within 6 months after injection of $10^{5}$ cells from wild type, PTP (Dox-treated), PTT (Dox-treated), and RPTP (Dox-treated) organoids, respectively. C, Graph showing the average tumor volume of mice injected with organoids of the genotypes indicated in $\mathbf{B}$. Data represent mean \pm $\mathrm{SE},{ }^{*} \mathrm{P}<0.01$. D, H\&E-stained sections of mammary fat pads of mice injected with $10^{6}$ cells from the indicated organoids at 3 months after transplant. Inserts show gross appearance of nodules/tumors. Scale bar, $50 \mu \mathrm{m}$. E, Left panel: exposed mouse abdomen 3 months after orthotopic injection of mice with $10^{6}$ PTPT organoid cells, showing large ovarian mass (circled on left panel) and widespread peritoneal metastasis (arrows). Right panel: the genital duct dissected from the left panel shows large tumors on ovary. F, \% of mice injected with the PTT (dox) organoids $(0 \%, n=10)$ and PTPT (Dox) organoids $(30 \%, n=10)$ that develop peritoneal metastasis, assessed at 6 months 
following injection of $10^{6}$ organoid cells. G, H\&E-stained sections and IHC staining for the indicated HGSOC markers in omental metastases. Scale bar, $50 \mu \mathrm{m}$.

Figure 4. Combined Tp53 mutation/RB family inactivation in Lgr5+ OSE also causes HGSOC-like malignancy. A, Scheme depicting Lgr5Cre;Rosa26-tdTomato mice.B, EGFP co-immunostaining of Tomato+ OSE clone in ovary and fallopian tube sections, $48 \mathrm{~h}$ or 4 months post 4-OHT induction of Lgr5-Cre;Rosa26-tdTomato mice. Scale bars, $50 \mu \mathrm{m}$. C, Scheme depicting generation of $\operatorname{Lgr} 5 \mathrm{Cr} \mathrm{E}^{E R T 2} ; \mathrm{T12} 1$; Tp $53^{R 172 \mathrm{H} /}$ mice (LPT mice). D, Exposed abdominal cavity of an LPT mouse 11 months after 4-OHT treatment, showing marked abdominal distention due to ascites, large ovarian tumors and peritoneal studding. E, \% of mice of the indicated genotypes showing ovarian tumors at 18 months post 4-OHT induction. F, \% LT and LPT mice with peritoneal metastasis 18 months after 4OHT treatment. G, Representative p53, CK7 and PAX8 staining (by IHC) in metastatic tumor from an LPT mice.

Figure 5. Transplanted OSE organoids also can give rise to HGSOC. A, Representative serial images of an OSE organoid at the indicated times after seeding. Magnification: $\times 10$. Scale bar, $20 \mu \mathrm{m}$. B, Left panel, H\&E stain of an OSE organoid. Middle panel, Immunofluorescence staining for E-cadherin (E-cad, green) and Ki67 (red). Right panel, immunofluorescence staining for PAX8 in a mixed culture of FTE (FT) and OSE (O) organoids. Note that only FTE organoids are strongly PAX8+. Scale bar, $20 \mu \mathrm{m}$. C, Representative flow cytometric plot, showing gates used to purify $\mathrm{EGFP}^{\mathrm{hi}}$ and $\mathrm{EGFP}^{\text {neg }}$ populations from Lgr5-GFP OSE cells (Left panel), and bright field pictures, showing organoids that developed from FACS-purified cells (5,000 cells/well) cultured for 6 days (Middle panel). Right panel shows organoid-forming efficiency of the two populations. ( $n=10$ wells per group combined from three experiments). Data indicate means \pm SEM, $* * * p<0.001$. D, Representative immunoblot for T121 in LPT organoids without 4-OHT treatment and 2 weeks after 4-OHT induction. ERK2 serves as a loading control. WT, wild type organoid. E, Micrographs showing typical OSE organoids from mice of the indicated genotypes after 6 days in culture. F, Relative organoid-forming efficiency of OSE cells from mice of the indicated genotypes. Cells (5000) were seeded from the $3^{\text {rd }}$ passage of each genotype, and organoids were counted at day 6 of culture. For each genotype, 3 independent mice were used to generate organoids. Data represent means \pm SEM. Statistical analysis was performed by Dunnett's multiple comparison test. ns, not significant. G, Growth curves of OSE organoids from the indicated mice. Note that cultures show exponential growth within the time window analyzed. Cells per well were counted at each passage from P1-P3 and P4-P6. Organoids from 3 independent mice for each group were used, each in duplicate. Data represent means \pm SEM. Statistical analysis was performed by Dunnett's multiple comparison test. $* * \mathrm{P}<0.01, * * * \mathrm{p}<0.001$. H, Scheme depicting OSE organoid transplantation (I and $\mathbf{J})$ Representative H\&E staining and IHC for the indicated markers in LPT organoids (top) and the orthotopic tumors derived from these organoids, 4-months post injection of $10^{6}$ cells (bottom).

Figure 6. Comparative transcriptome analysis of mouse and human HGSOC. A, Heatmap of sample distances with hierarchical clustering based on the overall gene expression levels of normal FTE (N-FT), normal OSE (N-O), and tumors derived from FTE (T-FT) and OSE (T-O), respectively. The cell shading represents the Euclidian distance for each sample pair. B, Venn diagram showing number of differentially expressed genes (adjusted $\mathrm{P}<0.05)$ in $\mathrm{T}$ FT vs N-FT, N-O vs N-FT, T-O vs N-O, and T-O vs T-FT. C and D, Significant KEGG pathway (C) and GSEA (D) analyses of the top 250 differentially expressed genes (DEGs) between the indicated groups $\mathbf{E}$, Application of Ovarian or Fallopian tube (O/FT) enrichment score, based on mouse Top 100, 250 and 500 DEGs (T-O or T-FT, respectively), to TCGA samples. Color coding indicates the transcriptional subtype assigned to each sample by TCGA. See Methods for details. T-FT and T-O signatures were compared across HGSOC molecular subgroups. 
The T-FT score was highest in the mesenchymal subgroup (upper panel); the T-O signature was highest in the proliferative subgroup (lower panel). Statistical analysis was performed by Dunnett's multiple comparison test. $* \mathrm{p}<0.05, * * * \mathrm{p}<0.001$.

Figure 7. Differential response of FTE- and OSE-derived tumor organoids to chemotherapy. Representative pictures and dose-response curves for LPT (OSE) and PTPT (FTE) organoids, treated with: A, Olaparib (0-50 $\mu \mathrm{M})$, B, Niraparib $(0-50 \mu \mathrm{M}), \mathbf{C}$, Gemcitabine $(0-500 \mathrm{nM})$, D, Carboplatin $(0-90 \mu \mathrm{M})$ or E, Paclitaxel $(0-30 \mu \mathrm{M})$ on. Cell viability was calculated relative to $0.01 \%$ DMSO-treated control cells, measured at 5 days of treatment. Each time point represents the means $\pm \mathrm{SEM}$ of 3 independent experiments, each in triplicate. ${ }^{*} \mathrm{p}<0.05, * * * \mathrm{p}<0.001$. 


\section{SUPLLMENTARY FIGURE LEGENDS}

Supplementary Figure 1: Ad-Cre injected into the ovarian bursa targets OSE and FTE. A, Schematic showing AdCre injection into ovarian bursa. B, X-gal staining shows $\beta$-galactosidase expression in OSE (left) and FTE (right) following injection.

Supplementary Figure 2: Lineage tracing of $\operatorname{Pax8}$ cells in the mouse fallopian tube. A, Scheme depicting induction of adult Pax8-rtTA;tetO-Cre;Rosa26-tdTomato mice by treatment with Dox for 2 days, followed by sacrifice and tissue collection. B, Immunofluorescence staining for acetylated- $\alpha$-tubulin (yellow), PAX8 (green) and DAPI (blue) in mouse oviductal epithelium after 2-day Dox treatment. C, Scheme showing chase experiment. D, Immunofluorescence staining for acetylated- $\alpha$-tubulin (yellow) and PAX8 (green) in oviductal epithelium of Pax8rtTA;TetOcre;Rosa26-tdTomato mice, 60 days after Dox induction. The lower panels in (B) and (D) represent a higher magnification of the corresponding upper panel. DAPI-stained nuclei are shown in blue. Tomato (red), acetylated- $\alpha$-tubulin (yellow), PAX8 (green) and DAPI (blue) staining, with the overlap (Merge) shown in the right panels. Scale bars: $20 \mu \mathrm{m}$.

Supplementary Figure 3: "Leaky" expression of TetOCre in thymic epithelium of PTT or PTPT mice results in lethal hyperplasia. A, Survival curves of PTP, TT, PTT and PTPT mice. TT: TetOcre;T121. B, Representative morphology of thymi from PTP, TT, PTT and PTPT mice. c, Representative Tomato immunofluorescence in thymi from Pax8rtTA;TetOcre;Rosa26-tdTomato without Dox treatment. Slides were counterstained with DAPI. Scale bars: $10 \mu \mathrm{m}$.

Supplementary Figure 4: Effect of mouse genotype on FTE organoid size. A, Bright field views of FTE organoids from the indicated mice, cultured for 6 days. Each culture was established by seeding 5,000 FTE cells. B, Average diameter of organoids from the indicated genotypes, measured on day 6 of culture. Organoids from 3 wells were analyzed for each group. Data represent means $\pm \mathrm{SEM}$. ${ }^{*} \mathrm{P}<0.05$; $* * * \mathrm{P}<0.01$. C, Organoid-forming efficiency for FTE cells from the indicated genotypes, quantified at day 6 of culture, from organoids derived from 3 independent mice of each genotype. Data represent the mean \pm SEM. Statistical analysis was performed by Dunnett's multiple comparison test. ns, not significant.

Supplementary Figure 5: Salpingectomy does not prevent neoplasia in Ad-Cre-induced $T p 53^{R 172 H / f ;}$ T121 mice. A, Schematic showing experimental strategy. B, Micrograph showing gross morphology (Left panel) and H\&Estained section of female genital tract from salpingectomized $T p 53^{R 172 H / f l} ; T 121$ mice, injected with Ad-Cre (Right panel). Dashed yellow ovals show lack of fallopian tube in the female genital tract. $\mathbf{C}$, Representative H\&E stain and Ki67 IHC of section from Ad-Cre-injected $T p 53^{R 172 H / f l}$;T121 mice with or without (Sham) salpingectomy, assessed 3 months post injection. The yellow dashed line shows the border between tumor and underlying cells.

Supplementary Figure 6: Lgr5 + cells can initiate tumors with markers of HGSOC. A, Schematic depicting crosses of Tp53 ${ }^{\text {R172H/- }}$;T121; Lgr5Cre and Rosa26-tdTomato mice. B, Lineage tracing scheme for Lgr5Cre;Rosa26Tdtomato mice. C, Whole mounts of ovaries from LPT;Rosa26-Tdtomato mice induced with 4-OHT at 6 weeks, followed by the indicated chase times, showing Tomato (red) and DAPI (blue) fluorescence. In left panel, region within the top white box is magnified below. Note expansion of isolated Tomato + cells into Tomato+ clones. Scale bar, $50 \mu \mathrm{m}$. D, Corresponding H\&E-stained sections from ovaries in (C). The yellow dashed line shows the border between the OSE-derived tumor and underlying cells. E, Relative areas of Tomato+ OSE clones at the indicated 
times after 4-OHT induction ("chase times"). Clone size was determined by measuring the longest 'length' of a discrete Tomato + clone. Each circle represents a distinct Tomato + clone, with $n=3$ ovaries analyzed/time point, and a total of 180 clones counted. F, Quantification of hyperplastic clone size on the ovarian surface. Clone size was determined by measuring the longest 'length' of epithelial protrusions (tumor cells) from the border with stromal cells (yellow dash line in D). Twenty (20) cross-sections from 3 ovaries were counted for each time point. Data represent mean \pm SEM. ${ }^{*} \mathrm{p}<0.05,{ }^{*} \mathrm{p}<0.01$, and $* * * \mathrm{p}<0.001$. G, Representative IHC (p53, Ki67 and Stathmin1) and immunofluorescence (WT-1) staining for key HGSOC markers in OSE from LPT mice at the indicated times after 4-OHT induction, or without treatment.

Supplementary Figure 7: Lgr5-Cre only directs tumorigenesis in OSE. A, Ki67 IHC staining of OSE and FTE from an LPT mouse. Right panels are higher magnifications (20X) of the left image. B, Immunofluorescence staining for PAX8 (red) and $\beta$-catenin (green) in ovary and fallopian tube, DAPI (blue) was used as nuclear counterstain. Note that FTE stains strongly for PAX8, while OT is PAX8 negative. OT: OSE-derived tumor. FT: fallopian tube. Fim: fimbria.

Supplementary Figure 8: Contribution of lineage-dependent and -independent gene expression to differential gene expression in FTE- vs OSE-derived tumors. Heatmap of the 50, 100 and 1000 most significant differentially expressed genes in OSE-derived (T-O) and FTE-derived (T-FT) tumors. For reasons of space, gene names are shown only for the top 50 genes. Red colored genes are reported lineage-related genes.

Supplementary Figure 9: Additional pathway analyses comparing normal FTE and OSE and the tumors derived from each. A, GO categories analysis B, Gene set enrichment analysis (GSEA). The size of each circle represents the number of DEGs within each category; color coding indicates the significance level.

Supplementary Figure 10: Validation of ovarian and fallopian tube-derived tumor signatures. Signatures were developed based on the top 50, 100, 250, or 500 FTE- or OSE-derived tumor-specific genes, and compared with the mean scaled gene expression from normal FTE (N-FT), normal OSE (N-O), and FTE (T-FT)- or OSE (T-)derived tumors, as indicated. See Methods for details.

Supplementary Figure 11: Model showing approaches used to assess cell-of-origin of HGSOC. Upper Panel: Combined RB family inactivation (via T121 expression) and Tp53 mutation in Lgr5+ OSE cells, initiated by CRE activation (with 4-OHT), results in patchy areas of OSE transformation that expand, taking over the entire ovarian surface and eventually spreading to the peritoneal cavity. These processes can be reproduced by transplanting cognate OSE organoids. Lower Panel: The same genetic events also cause transformation of Pax $8+$ FTE, leading to Serous Tubal Intraepithelial Carcinoma (STIC) and ovarian metastasis. These behaviors also can be recapitulated in FTE organoids, which generate widespread abdominal metastasis following orthotropic injection.

Supplementary Table S1: RNA analyses. A, DEGs shared between N-O vs N-FT and T-O vs T-FT at Pvalue $<0.05$. B, Top50 DEGs between T-O (OSE-derived tumor) and T-FT (FTE-derived tumor), N-O (Normal OSE) and N-FT (Nomal FTE) at a fold change $(\mathrm{FC})>2(\log 2 \mathrm{FC}>1)$. C, DEGs in diferent comparisons in T-O vs N-O, T-FT vs N-FT and the shared DEGs between T-O vs N-O and T-FT vs N-FT. All at Pvalue $<0.05$. 
bioRxiv preprint doi: https://doi.org/10.1101/481200; this version posted November 29, 2018. The copyright holder for this preprint (which was not certified by peer review) is the author/funder. All rights reserved. No reuse allowed without permission.

Supplementary Table S2: Top 100, 250 and 500 T-FT and T-O specific mouse genes and the corresponsive HGSOC patient genes from TCGA.

Supplementary Table S3: Scores and $P$ values of multiple comparisons among T-FT and T-O enriched HGSOC subtypes. 
bioRxiv preprint doi: https://doi.org/10.1101/481200; this version posted November 29, 2018. The copyright holder for this preprint (which was

A

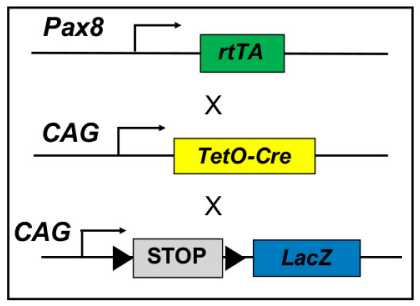

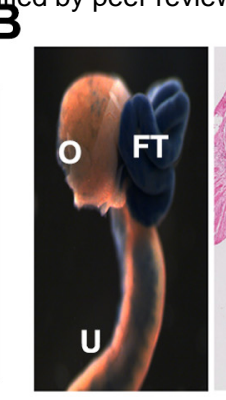

C

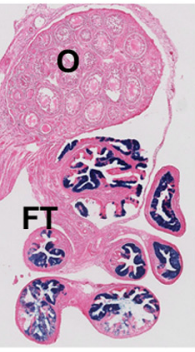

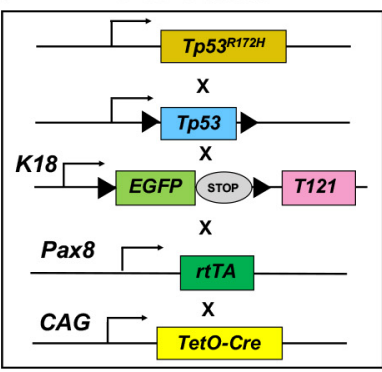
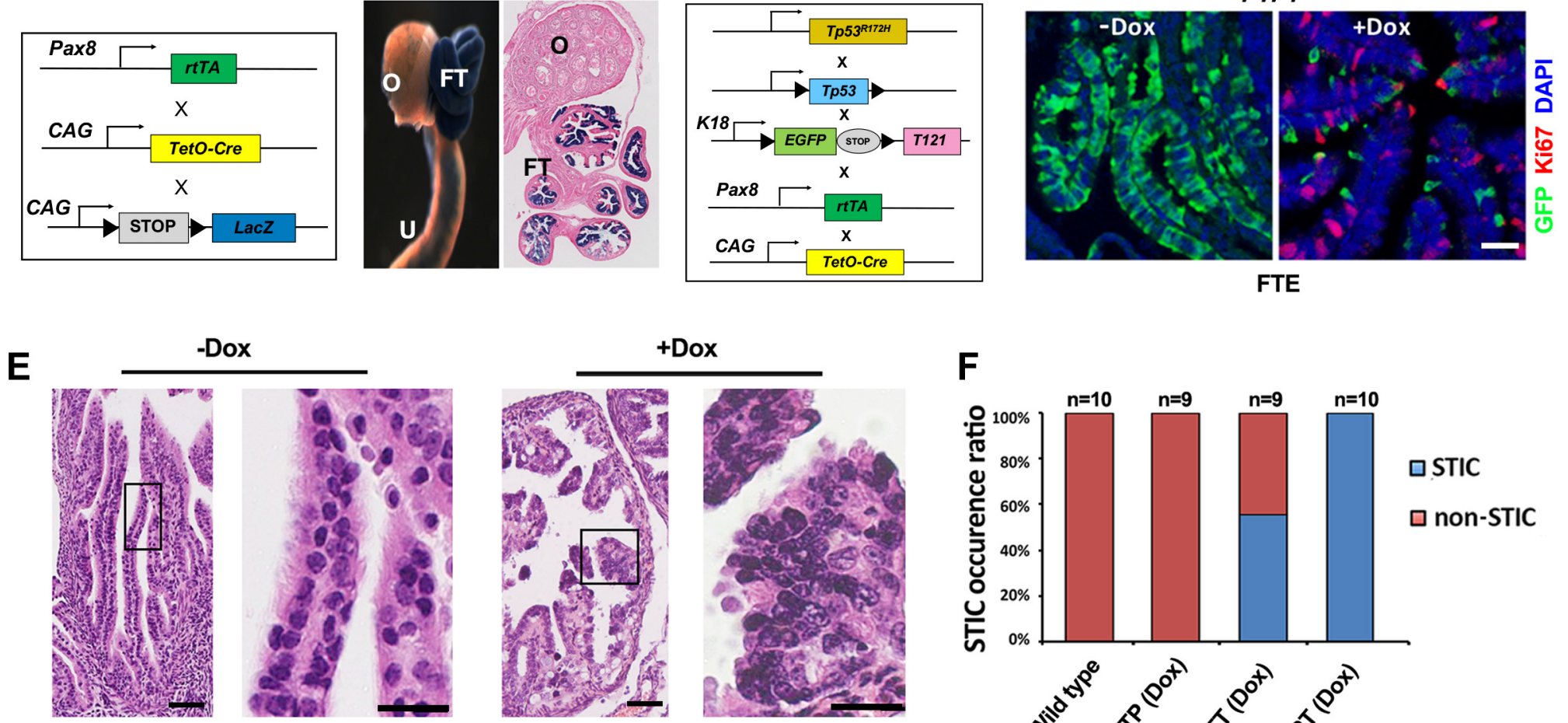

G

-Dox
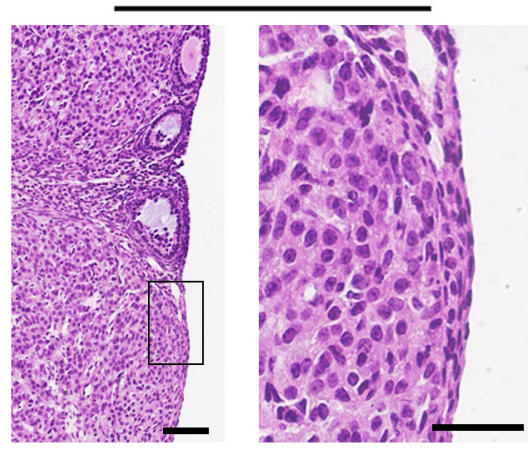

PTPT

$D^{2}$

FTE
F

H

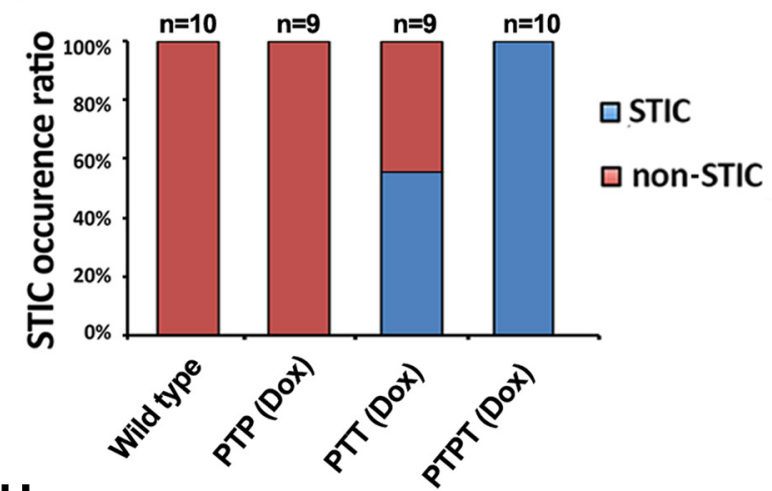

$\square$ metastasis

$\square$ no metastasis
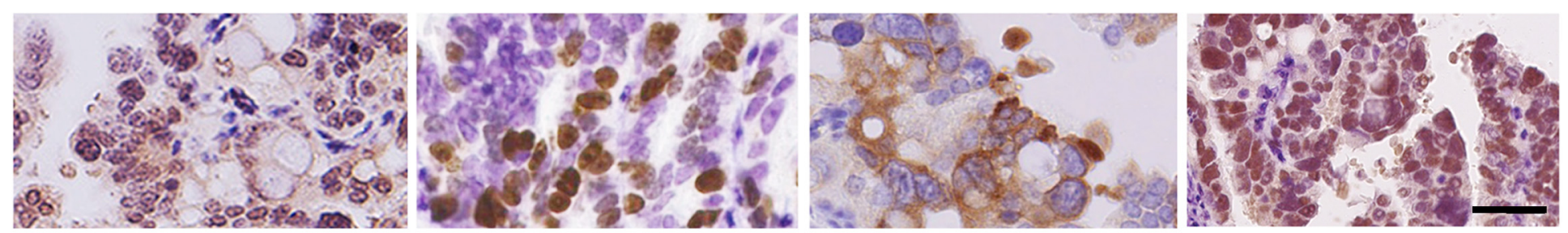

J

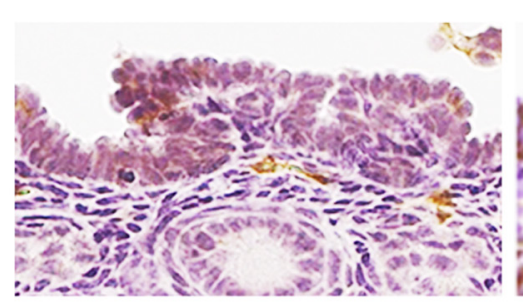

p53

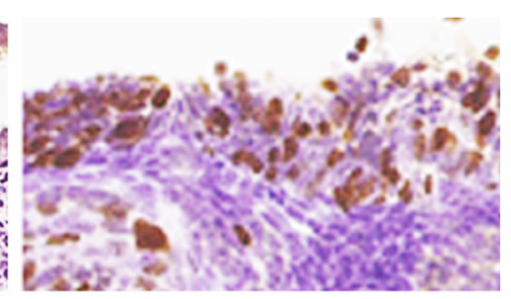

Ki67

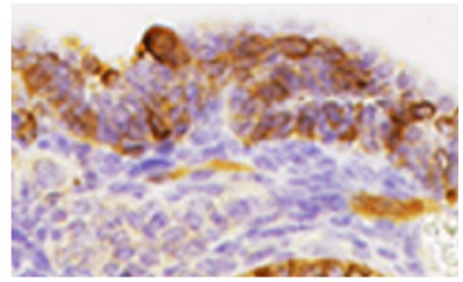

Stathmin1

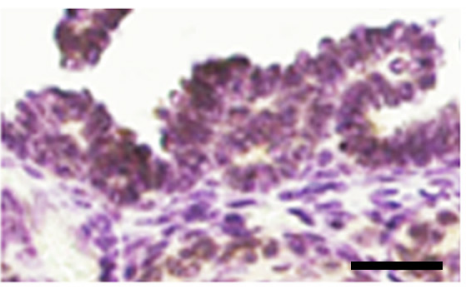

PAX8 


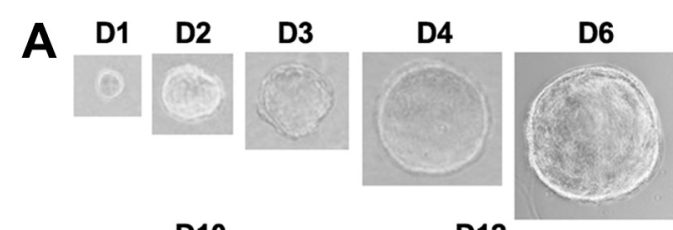

B
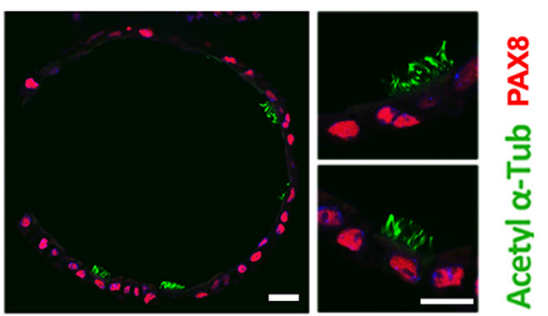

E

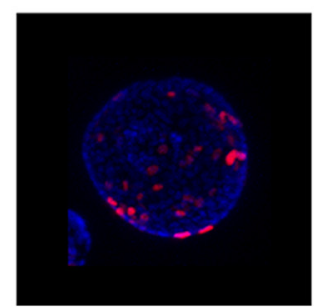

G

$$
\text { Wild type }
$$

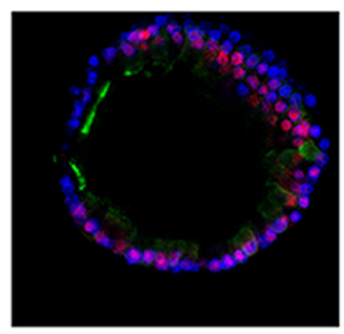

Wild type

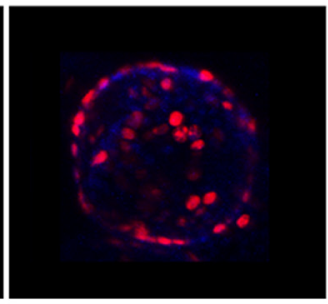

PTP (Dox)
C
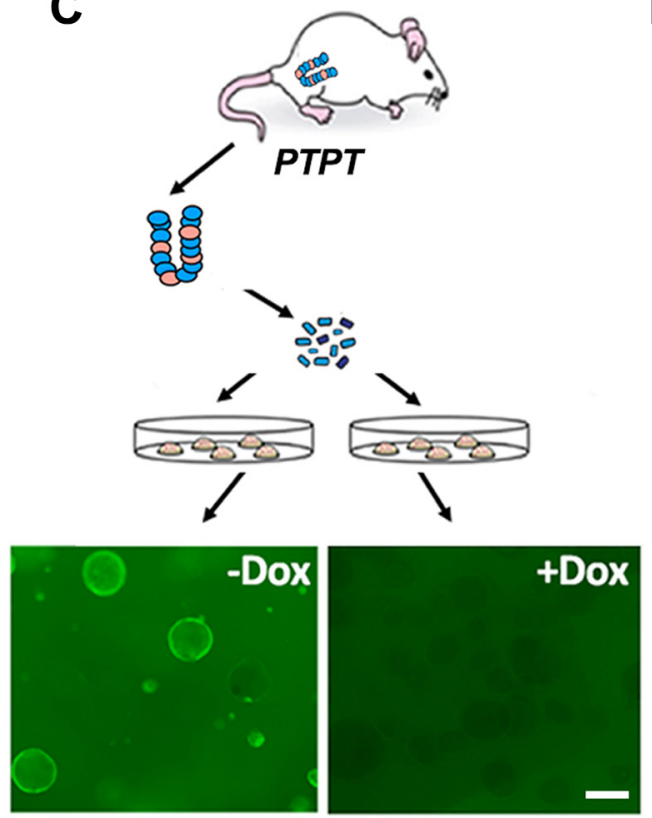

D
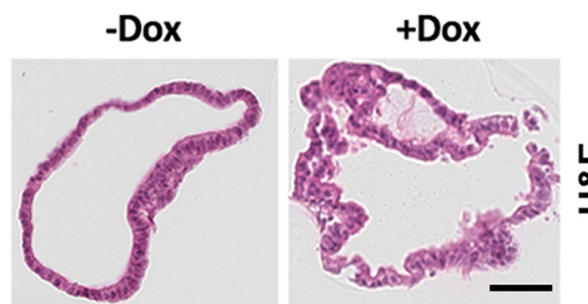

Ш
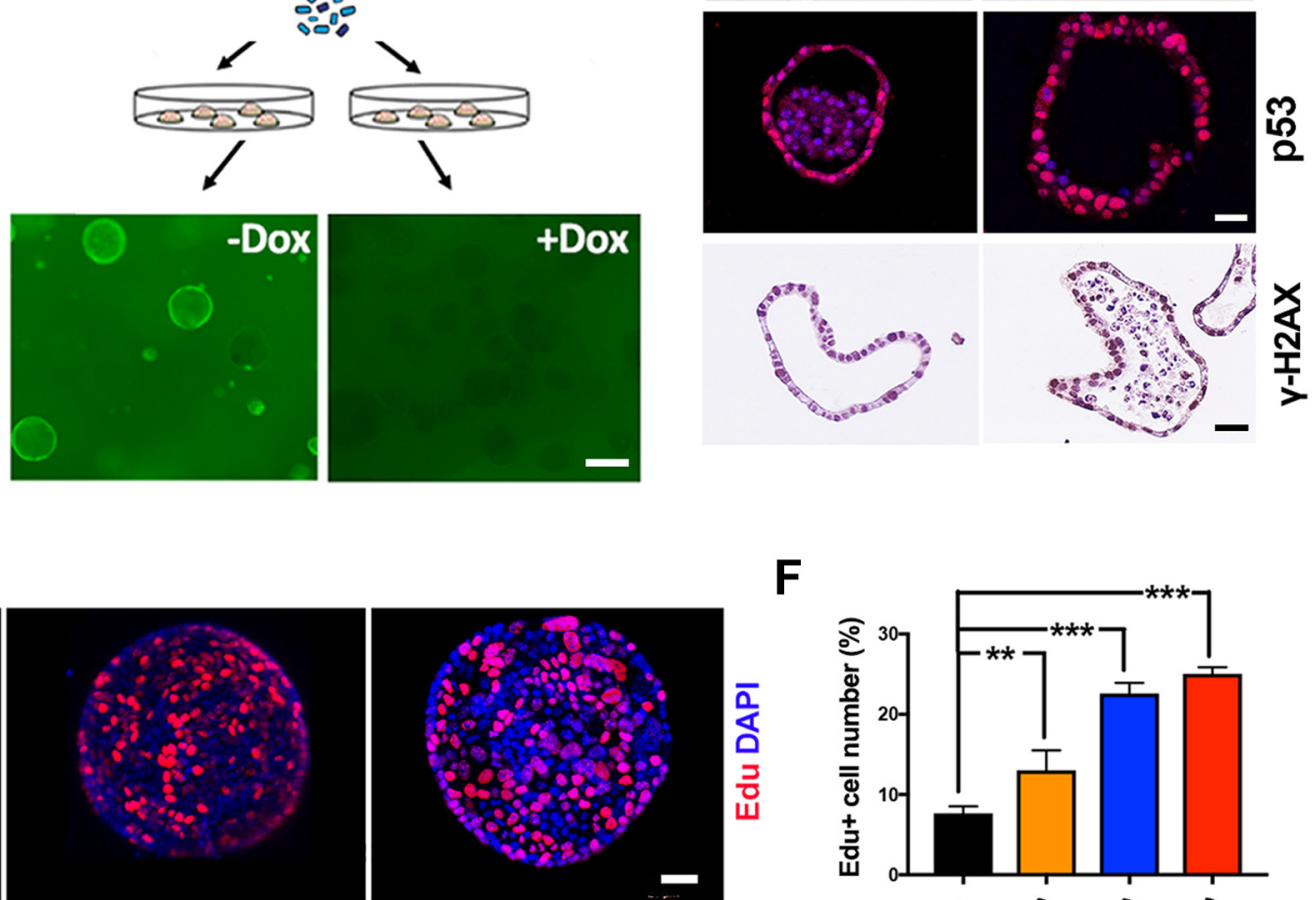

PTT (Dox)

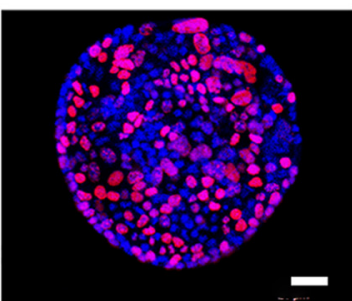

PTPT (Dox)

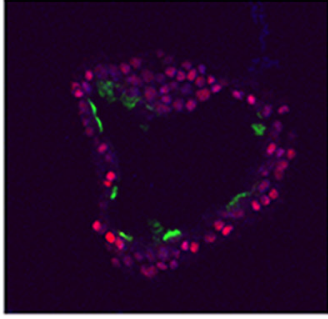

PTP (Dox)

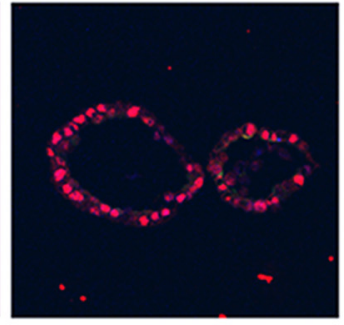

PTT (Dox)

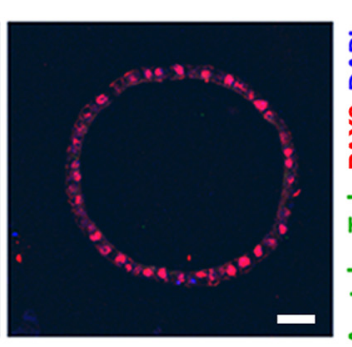

PTPT (Dox)

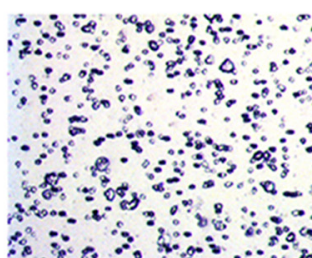

80

Wild type

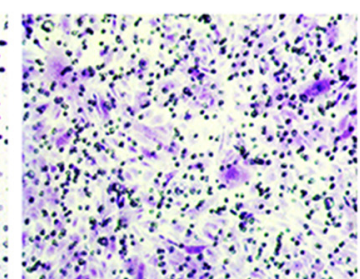

PTP (Dox)

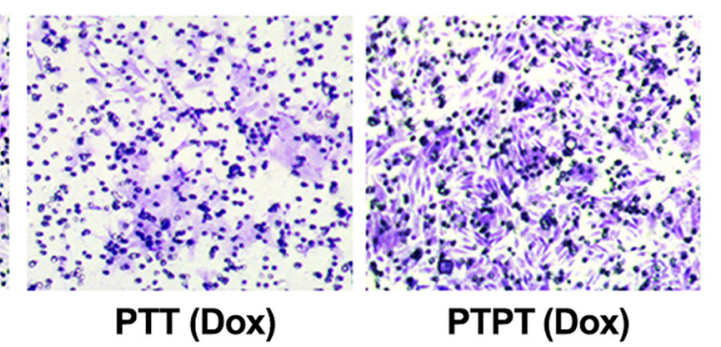

PTPT (Dox)
F
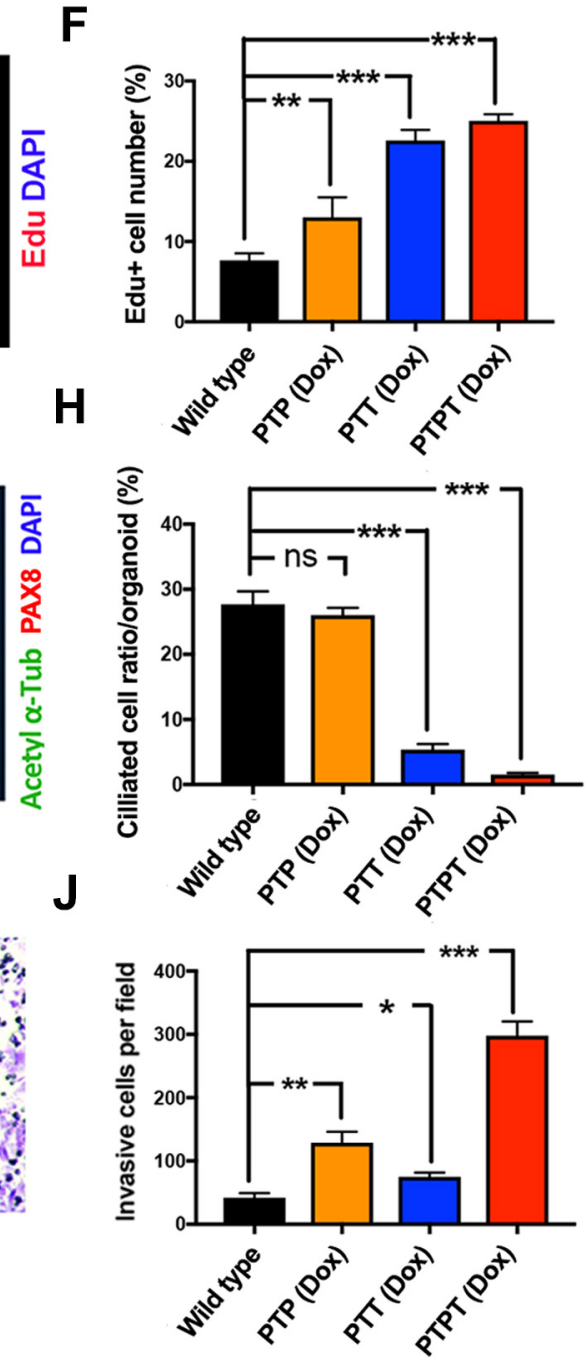
A

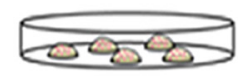

Expand mutant organoids

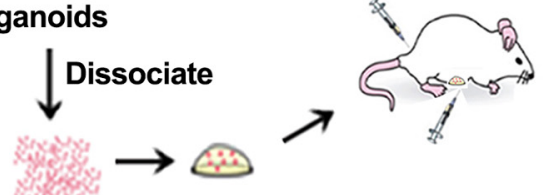

Mix with Matrigel

D

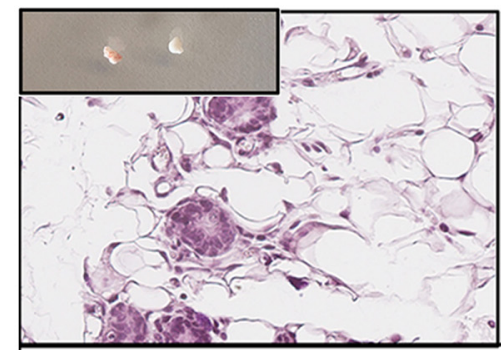

Wild type
B

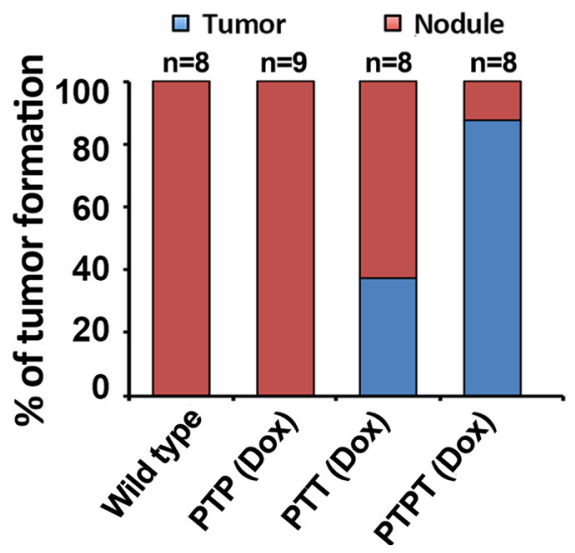

C

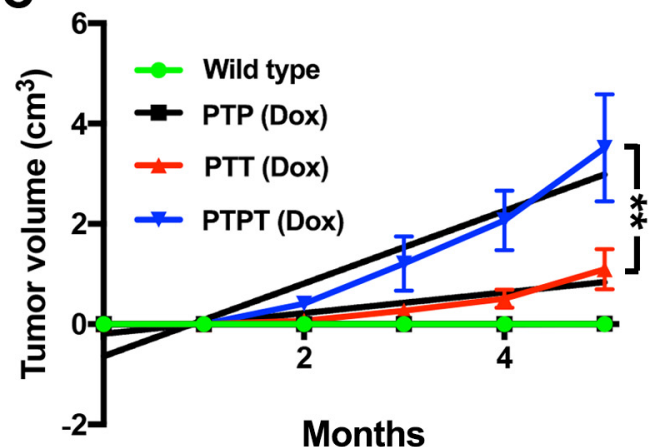

$\mathbf{E}$

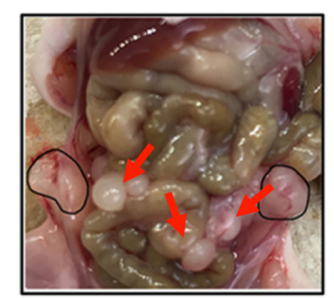

$\mathbf{F}$

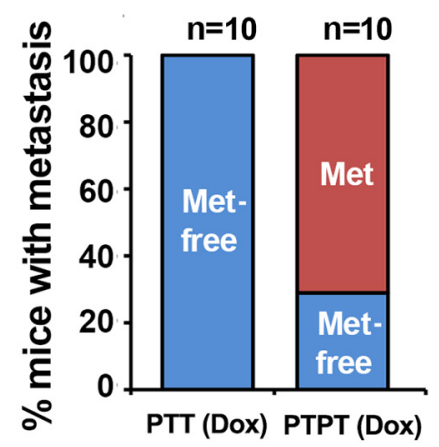

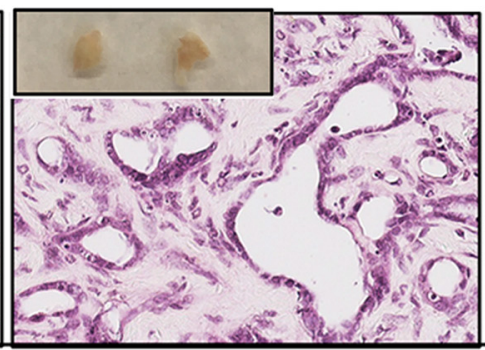

PTP (Dox)

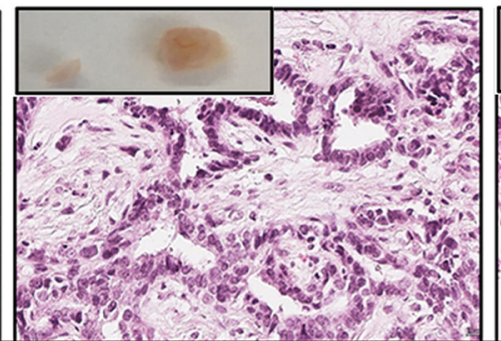

PTT (Dox)

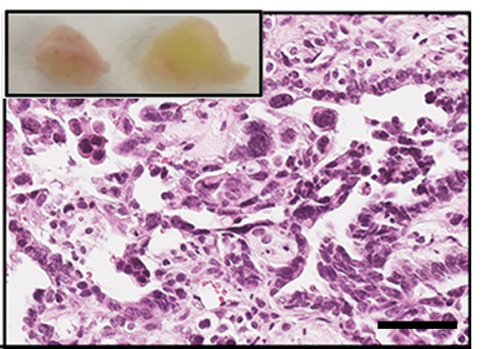

PTPT (Dox) 
A
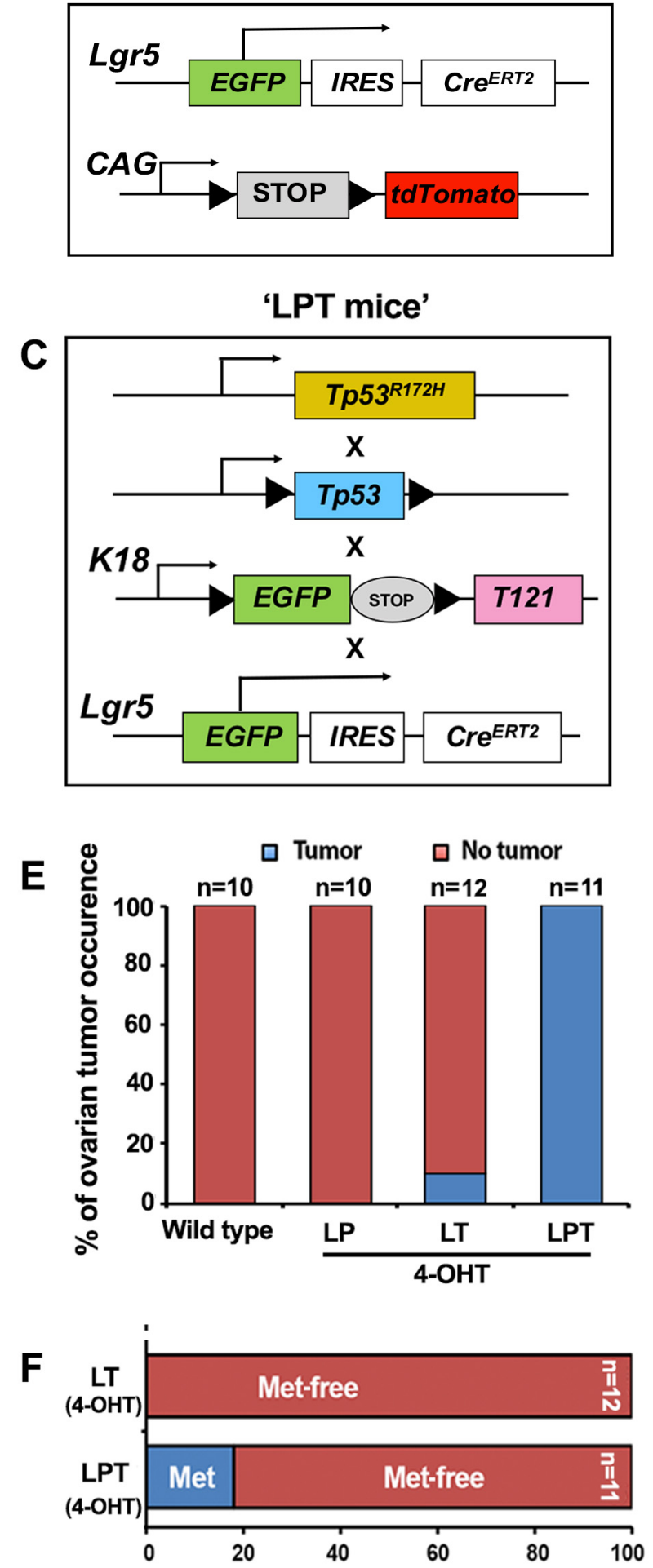

B
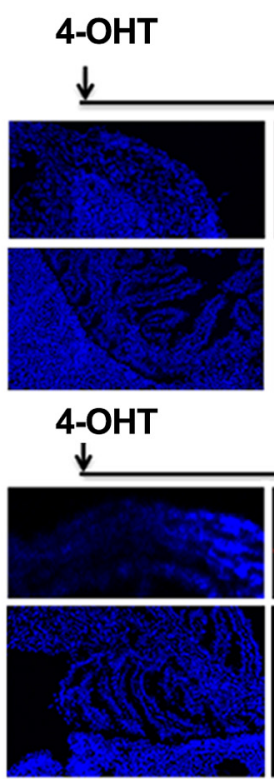

DAPI

D
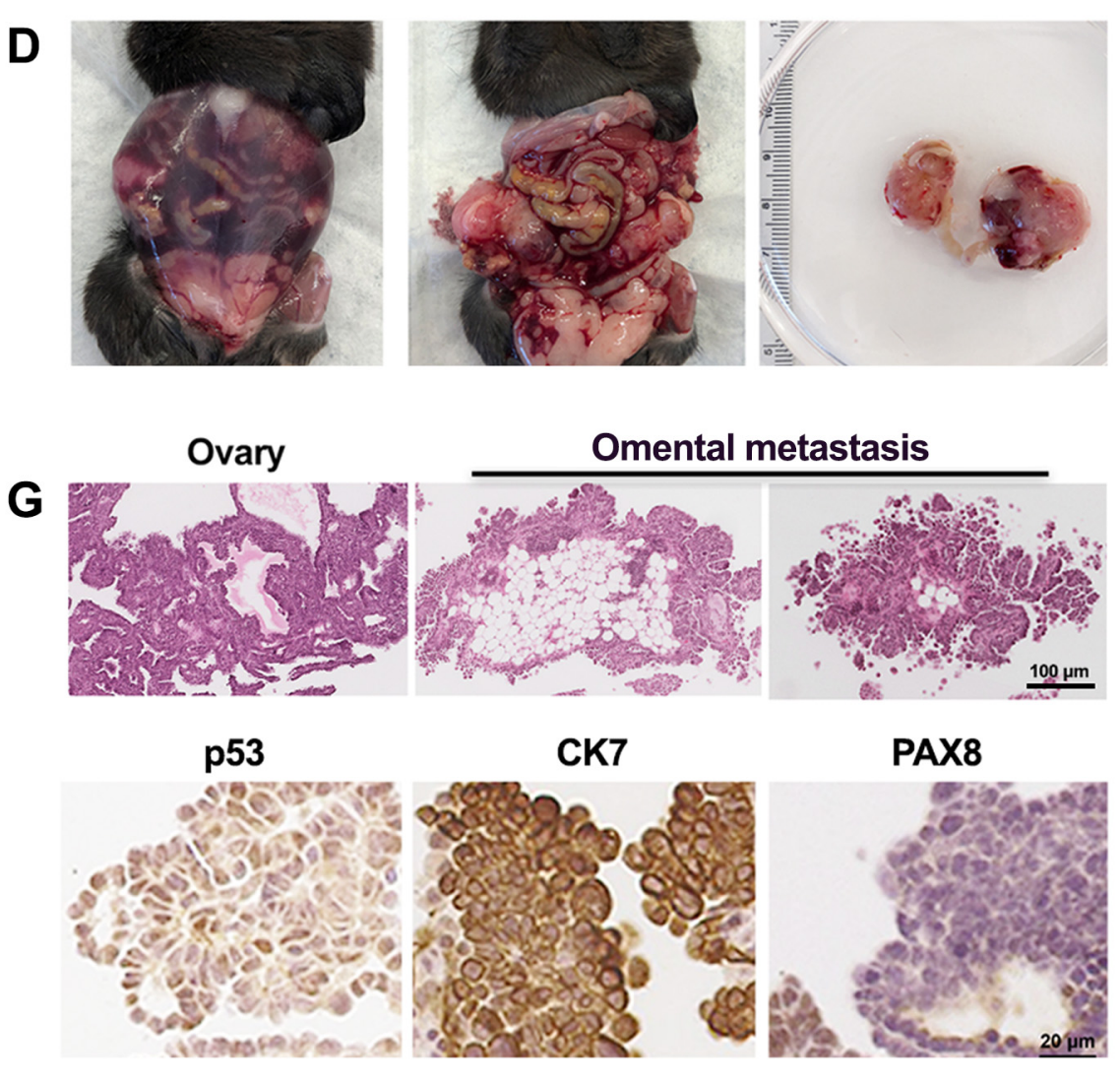

Figure 4 
A

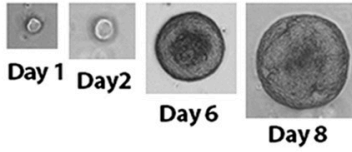

B

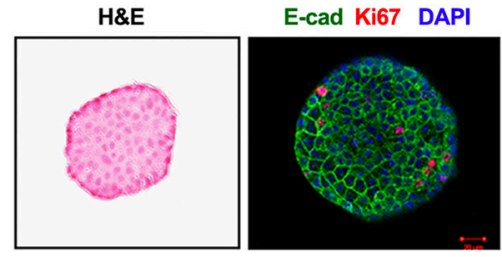

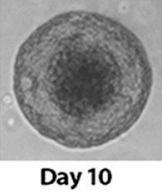

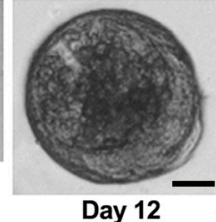

PAX8 DAPI

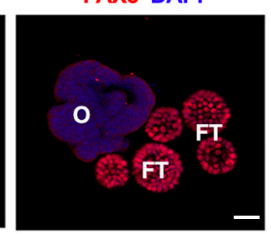

C

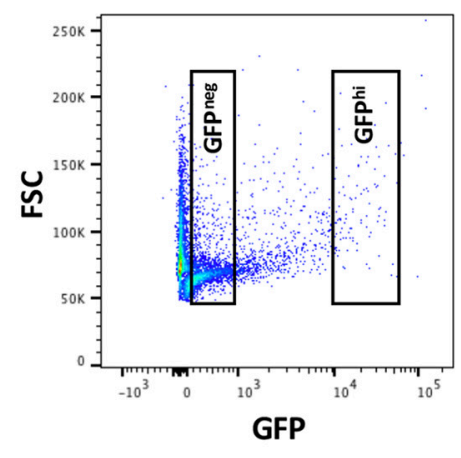

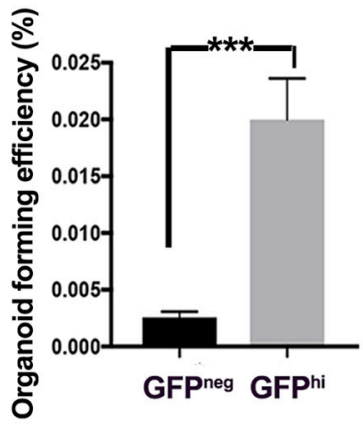
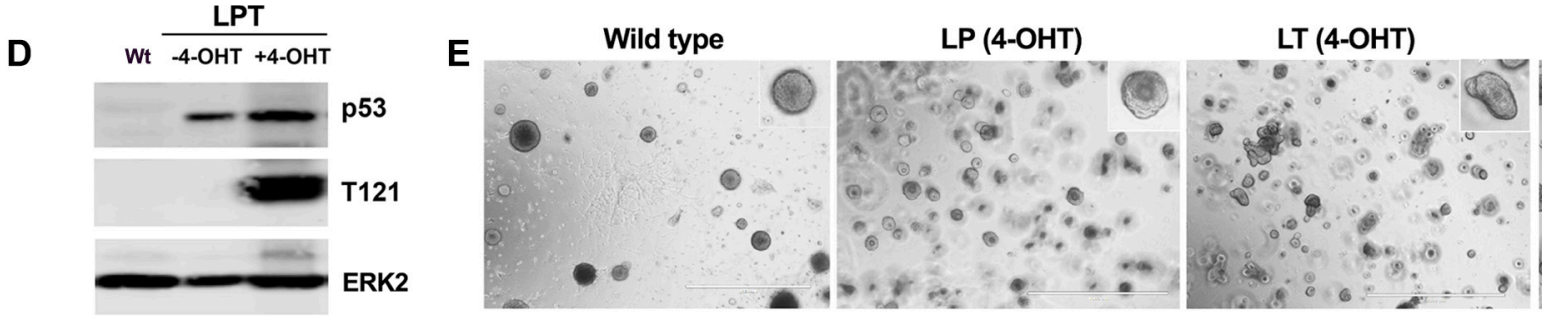

LPT (4-OHT)

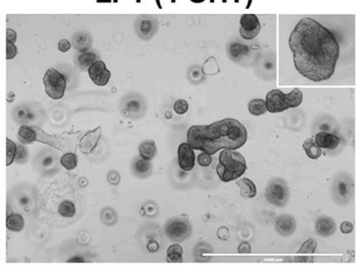

$\mathbf{F}$

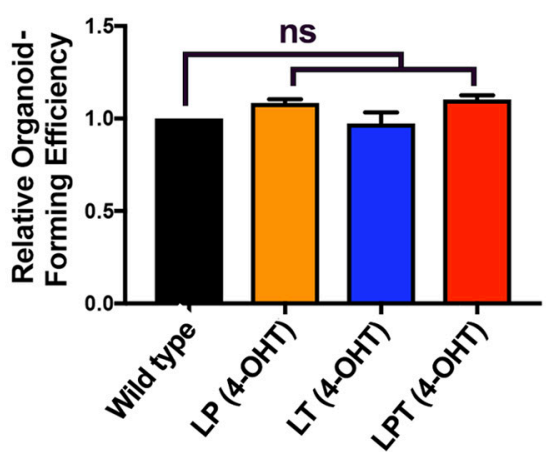

G $\left(x 10^{5}\right)$ Serially passaged (P0-P3)

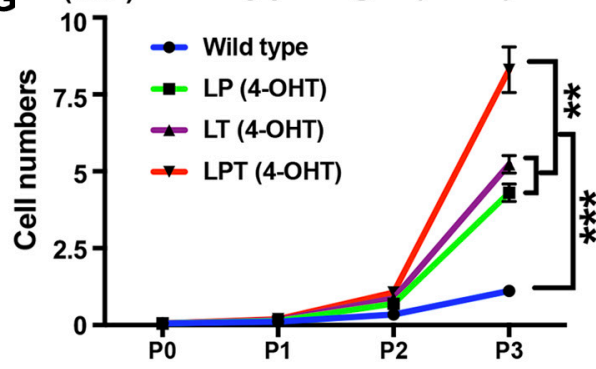

Passages (7 days/passage)

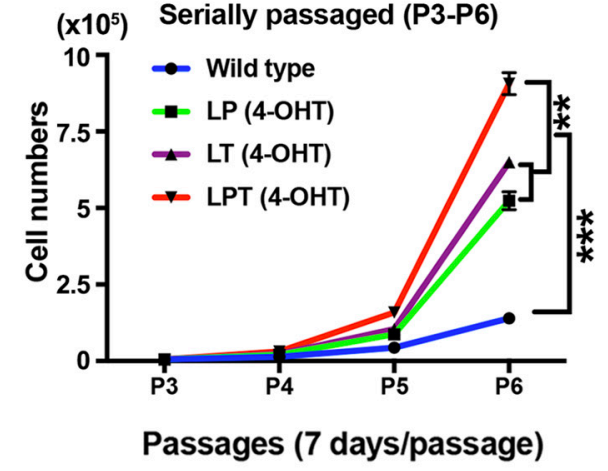

Passages (7 days/passage)

H

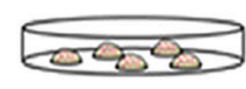

Expand mutant organoids $\downarrow$ Dissociate<smiles>CC1(C)CCCCC1</smiles>

Orthotopic injection
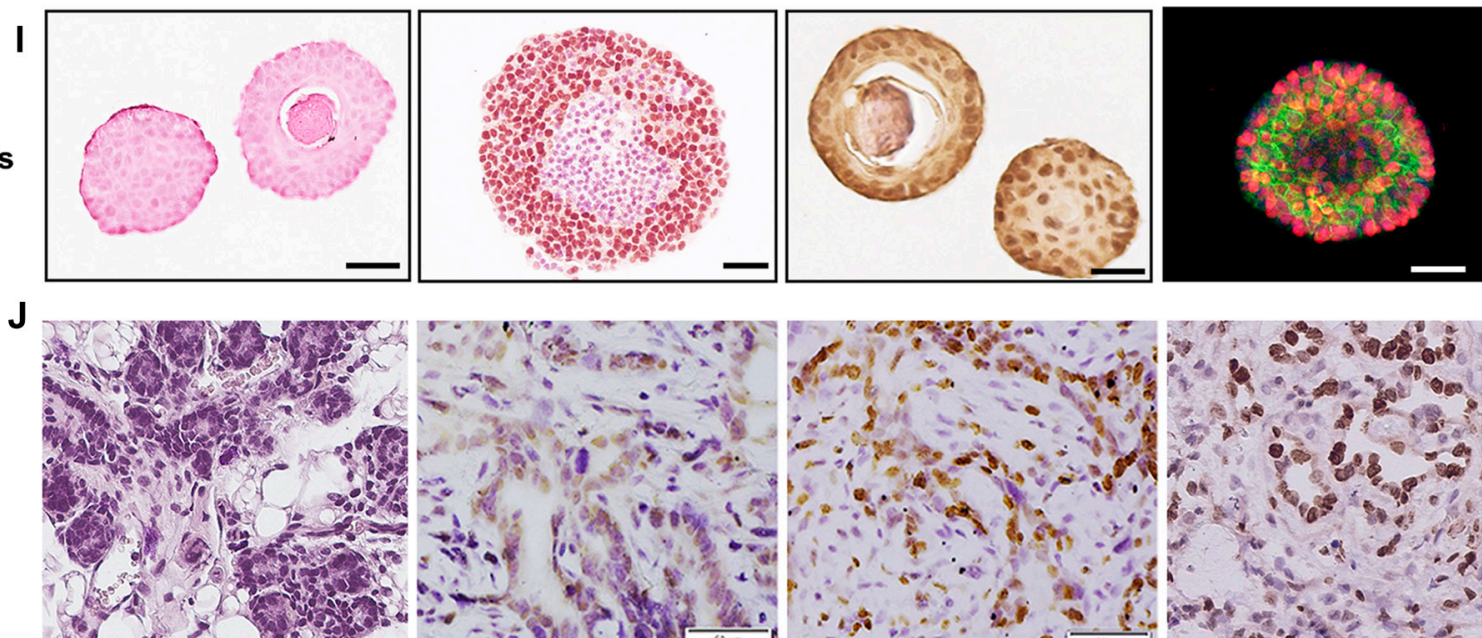

H\&E

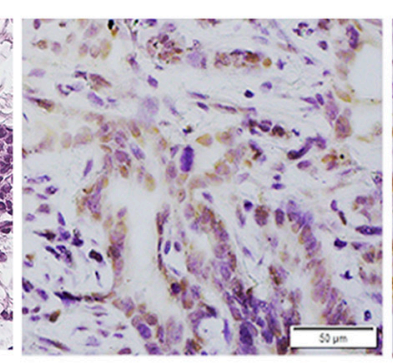

p53
WT1

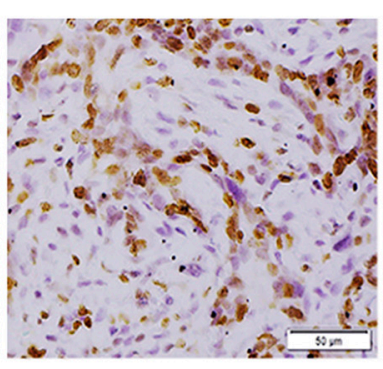

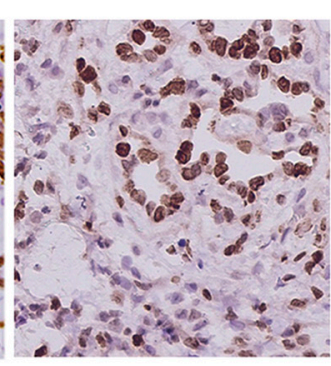

Ki67 
bioRxiv preprint doi: https://doi.org/10.1101/481200; this version posted November 29, 2018. The copyright holder for this preprint (which was
not certified by peer review) is the author/funder. All rights reserked, No reuse allowed without permission

A - ${ }_{0}$ not certified by peer review) is the author/funder. All rights resered, No reuse allowed without permission - OSE
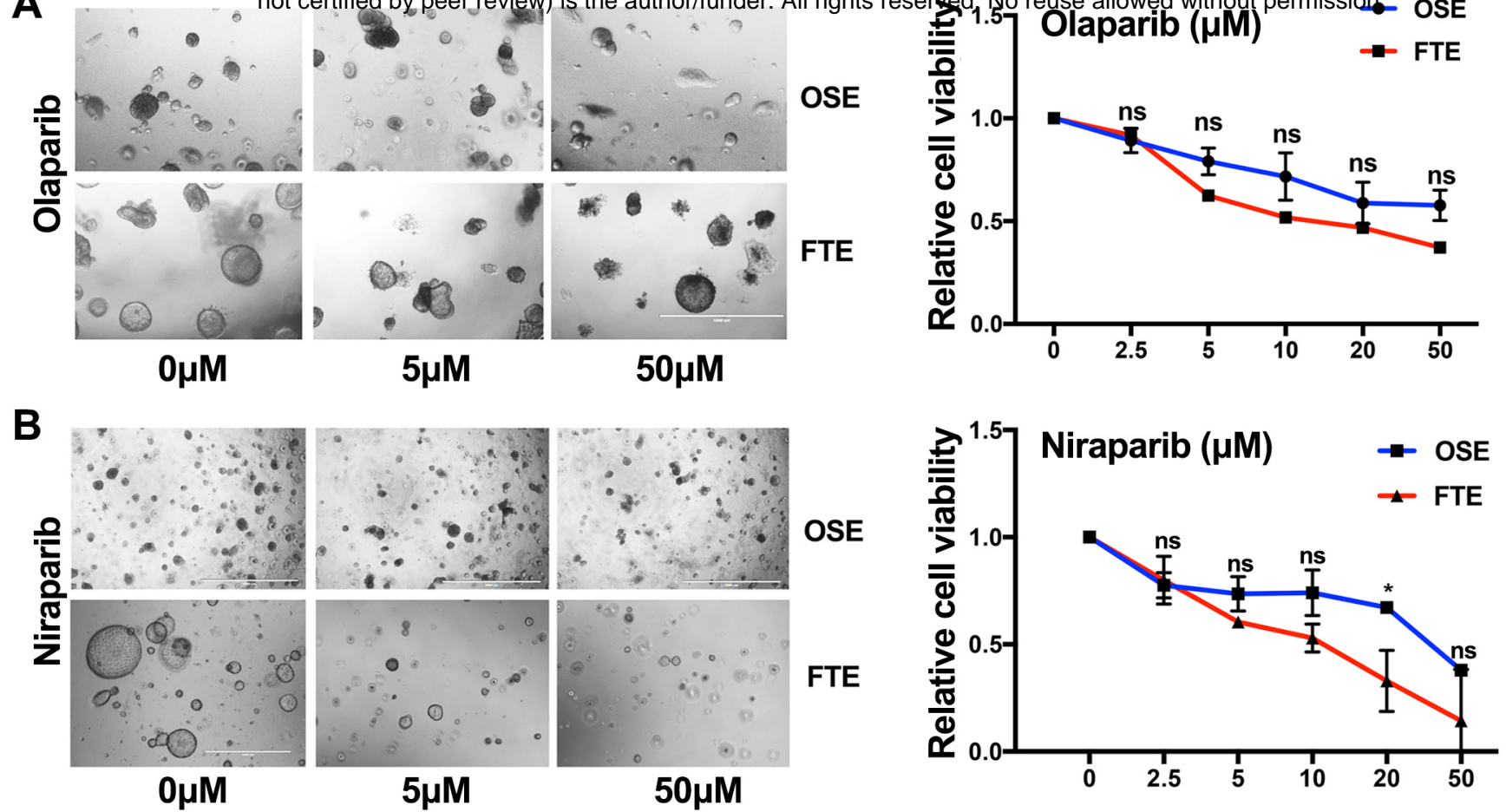

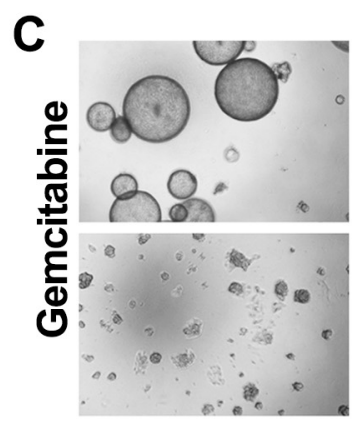

OnM

D

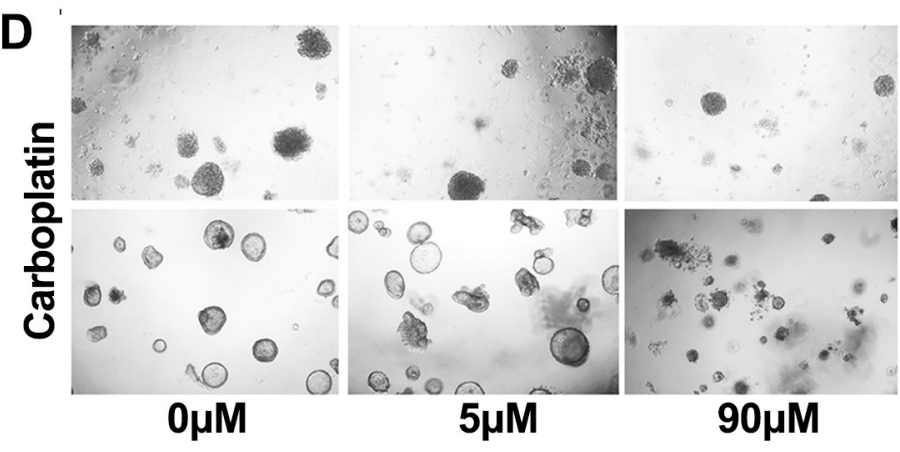

E
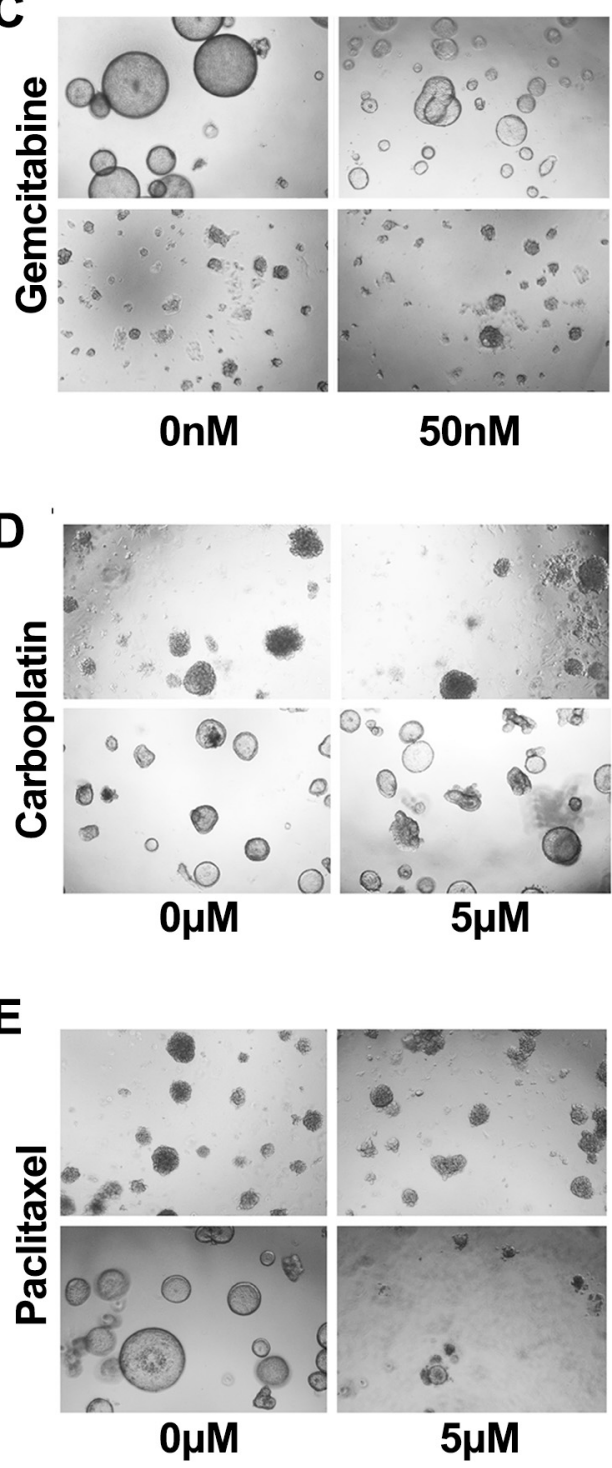

$50 \mathrm{nM}$

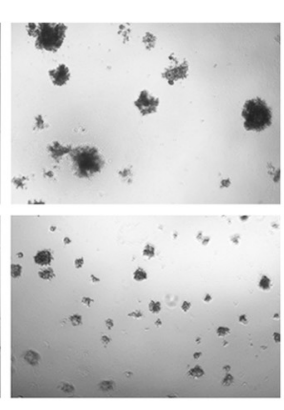

500nM

OSE

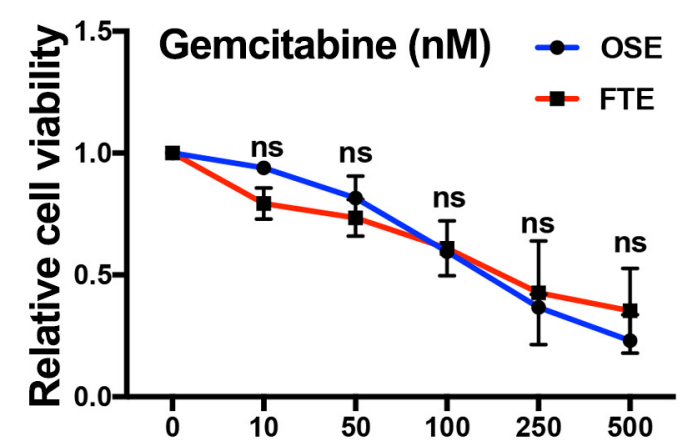

FTE
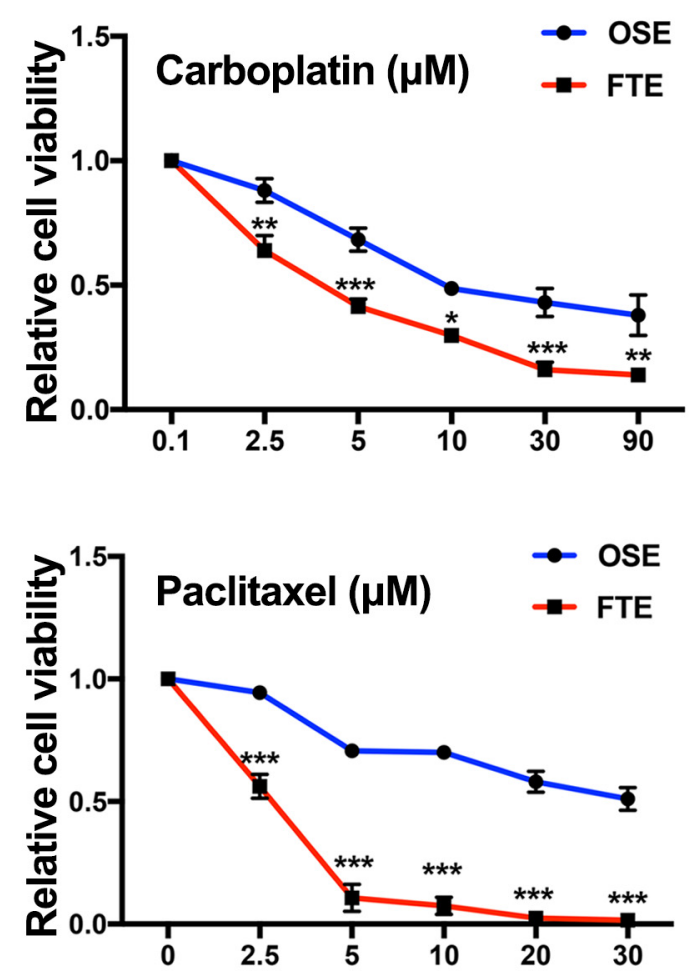
A

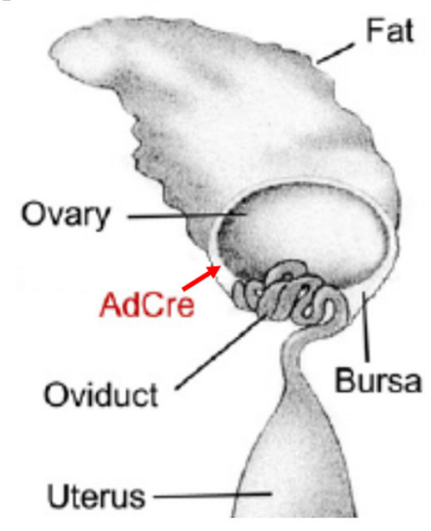

B

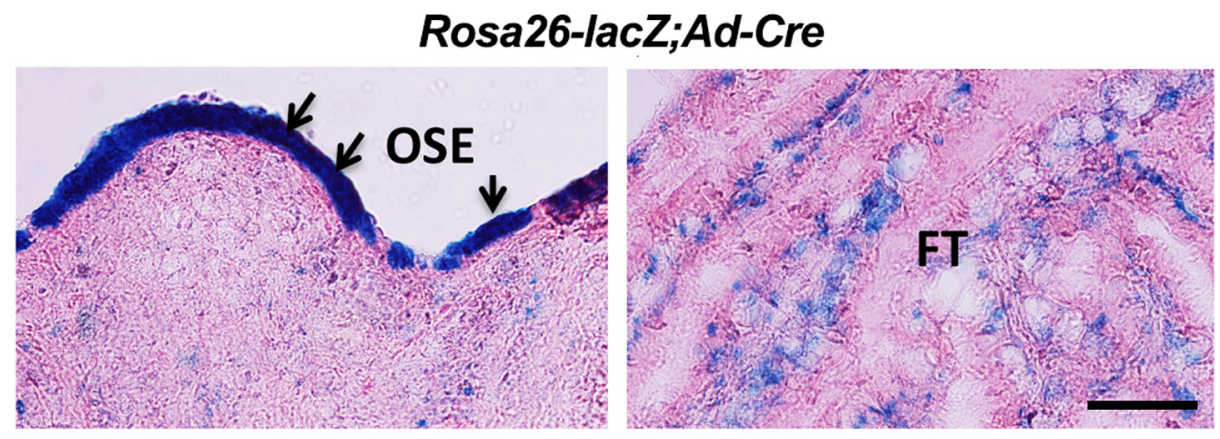


A

Pax8rtTA; TetOcre,Rosa26-tdTomato

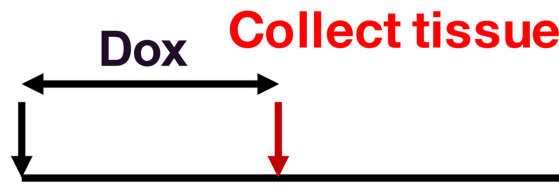

Day 0 Day 2

B

DAPI

PAX8
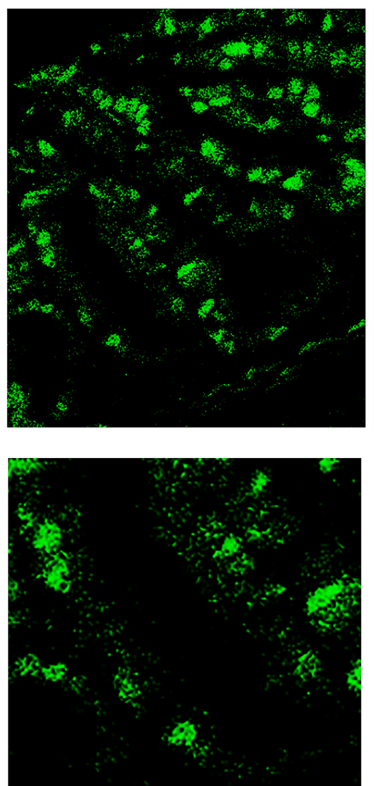

Tomato
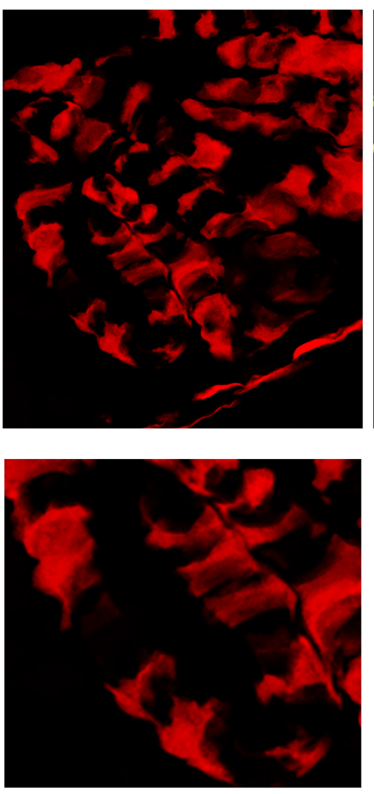

acetyl-or-Tulb
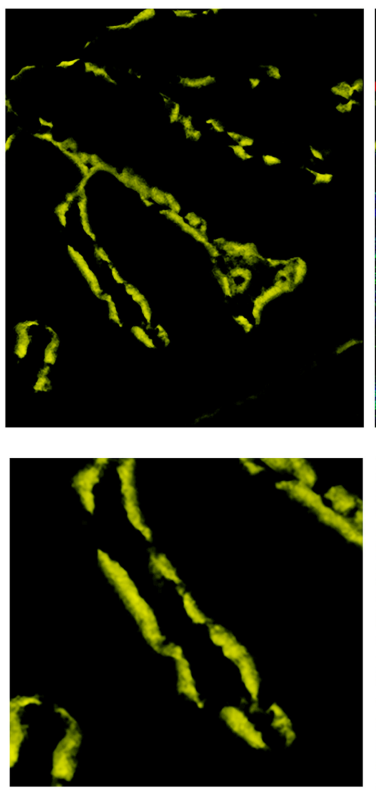

Merge
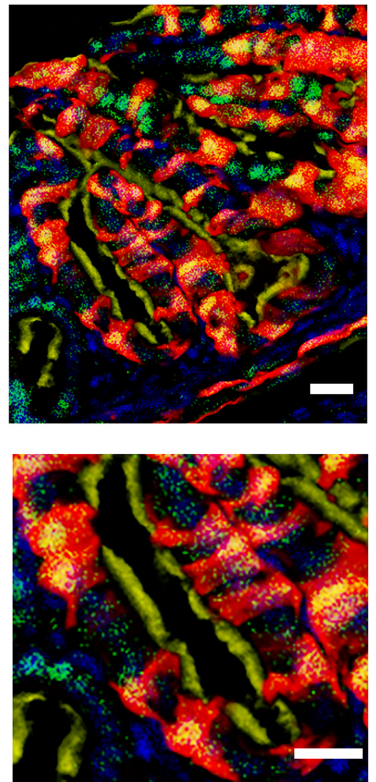

C Pax8rtTA;TetOcre;Rosa26-tdTomato

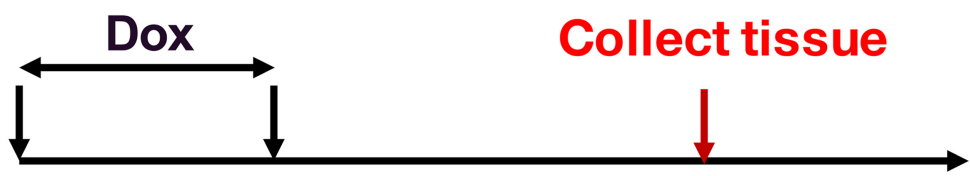

\section{$\begin{array}{lll}\text { Day } 0 & \text { Day } 2 & \text { Day } 60\end{array}$}

D
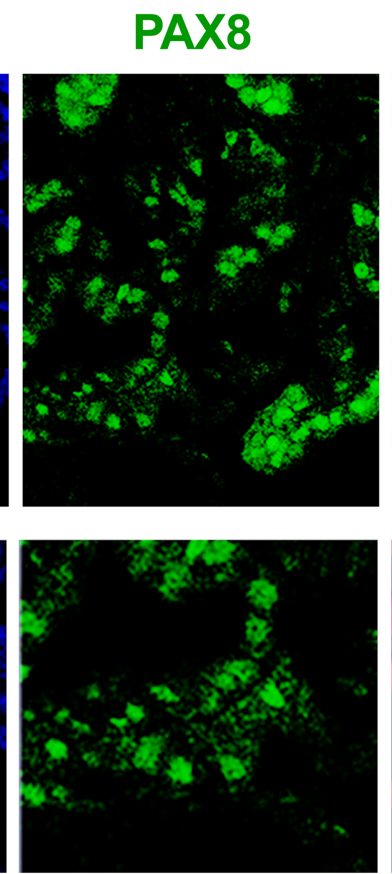

Tomato
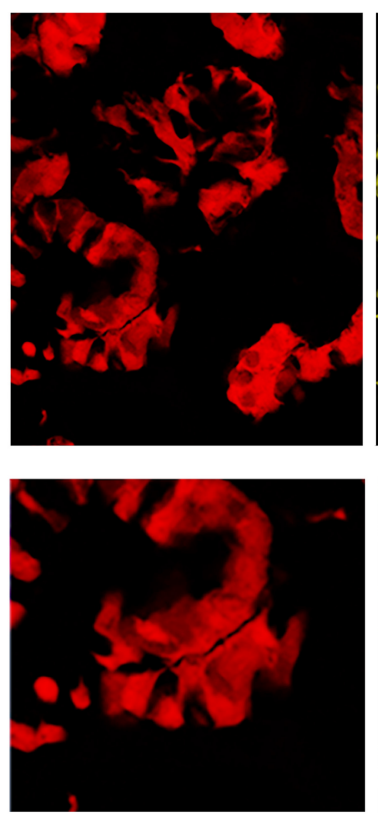

acetyl-a-Tulb
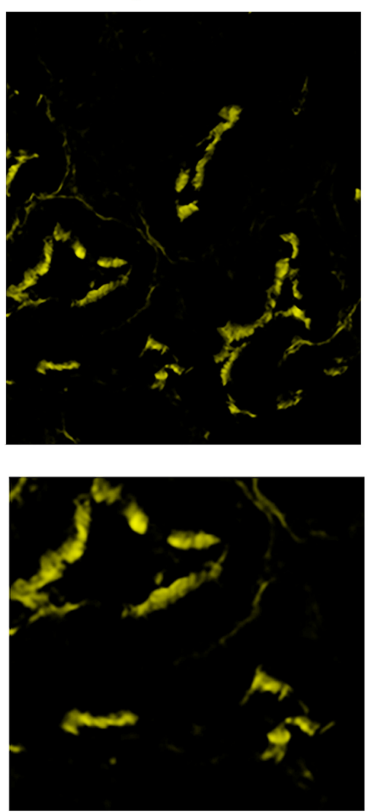
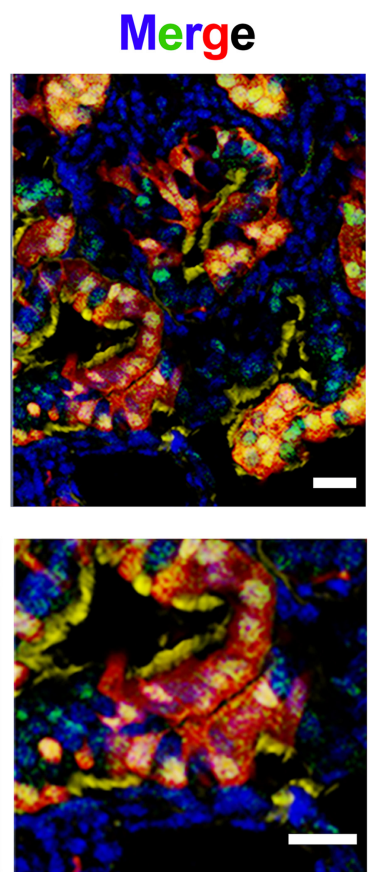
A

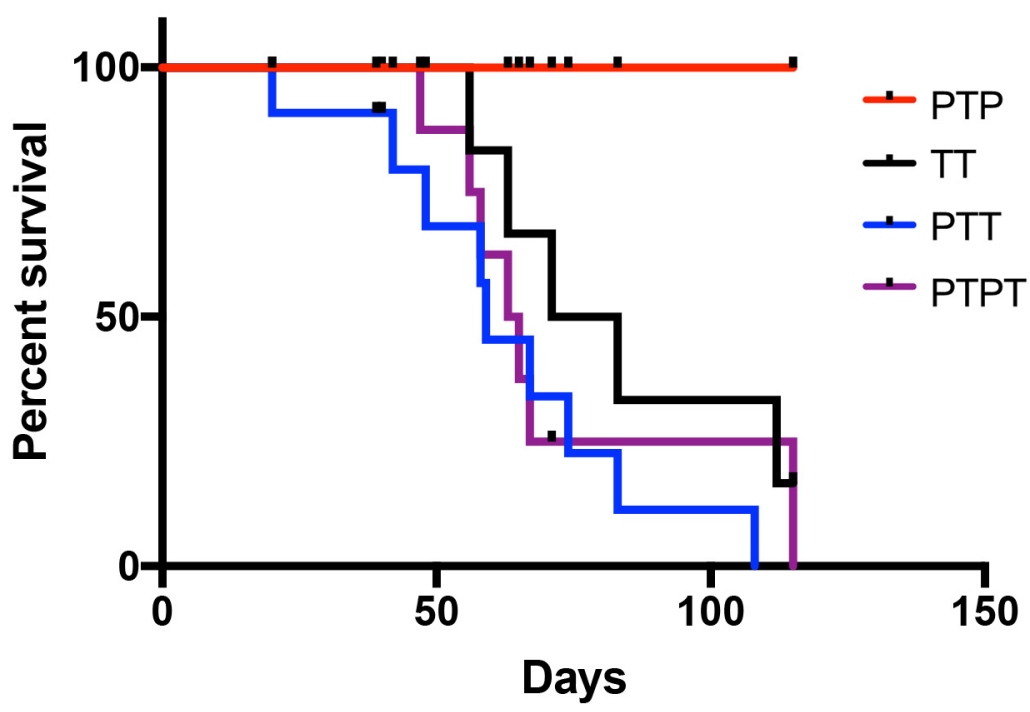

B
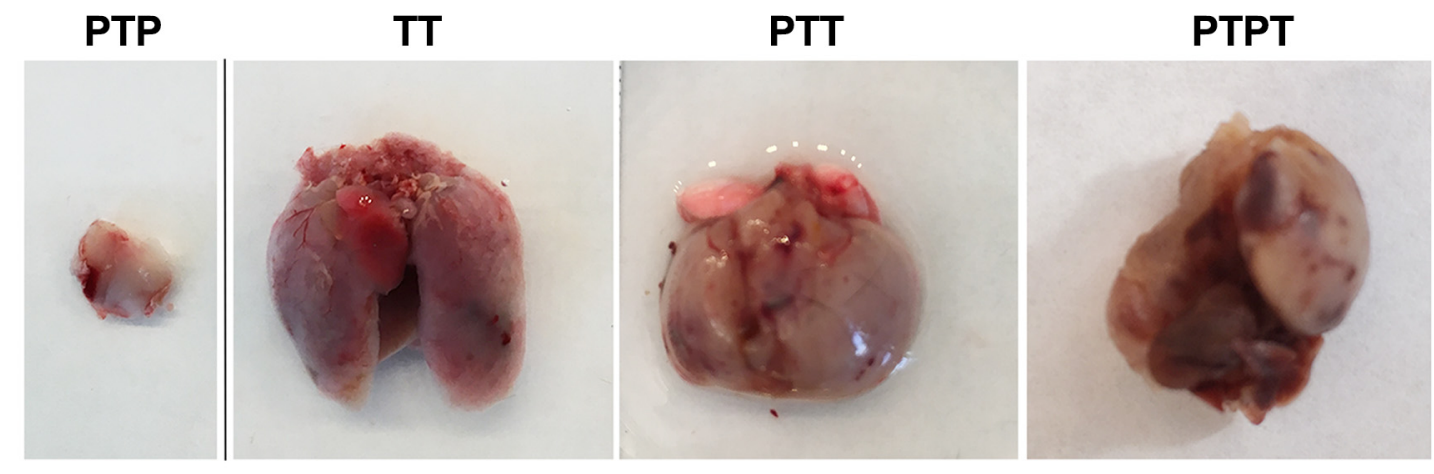

C
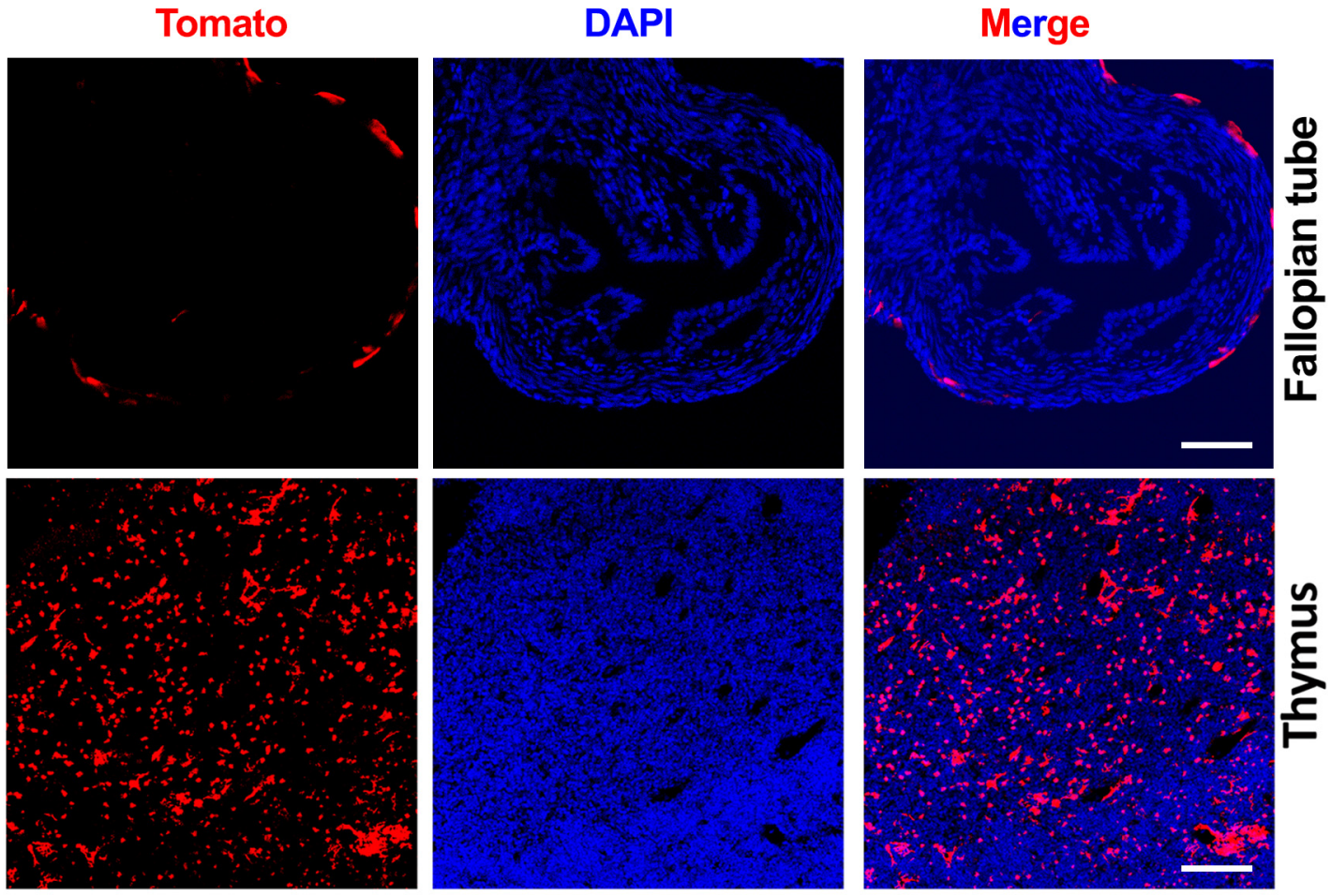

Pax8rtTA;TetOcre;Rosa26-tdTomato

\section{Supplementary Fig.3}


A
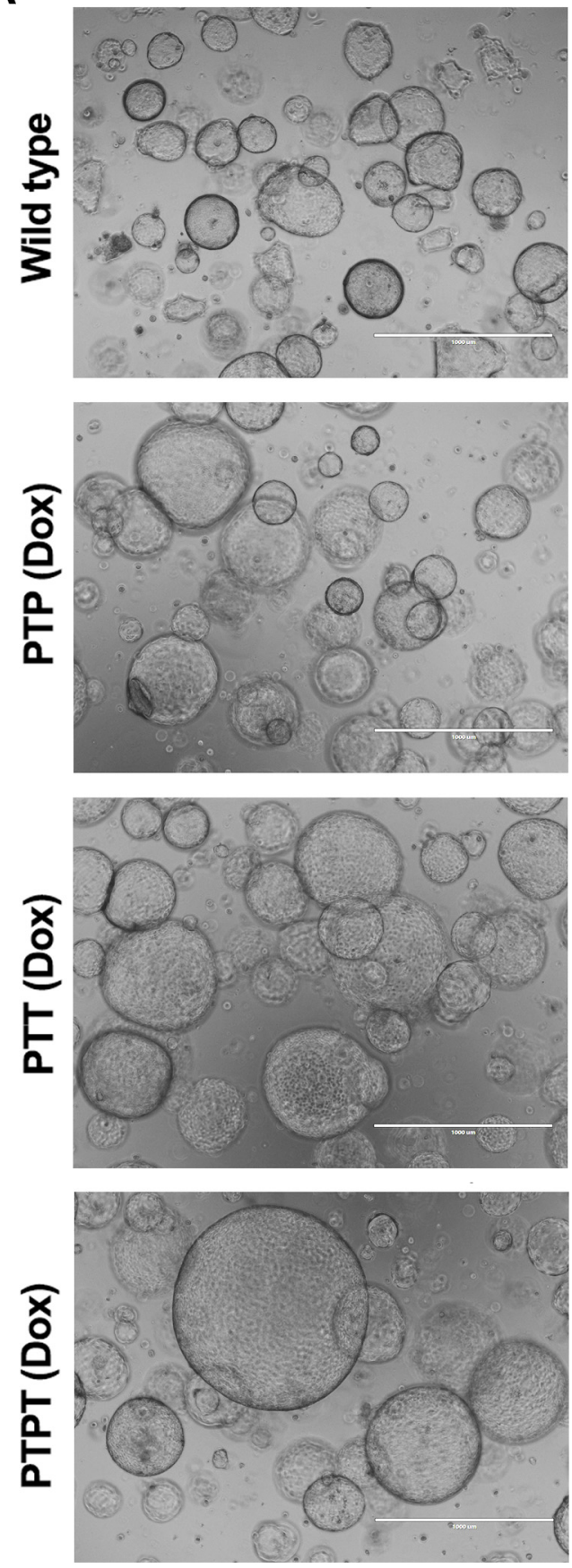

B

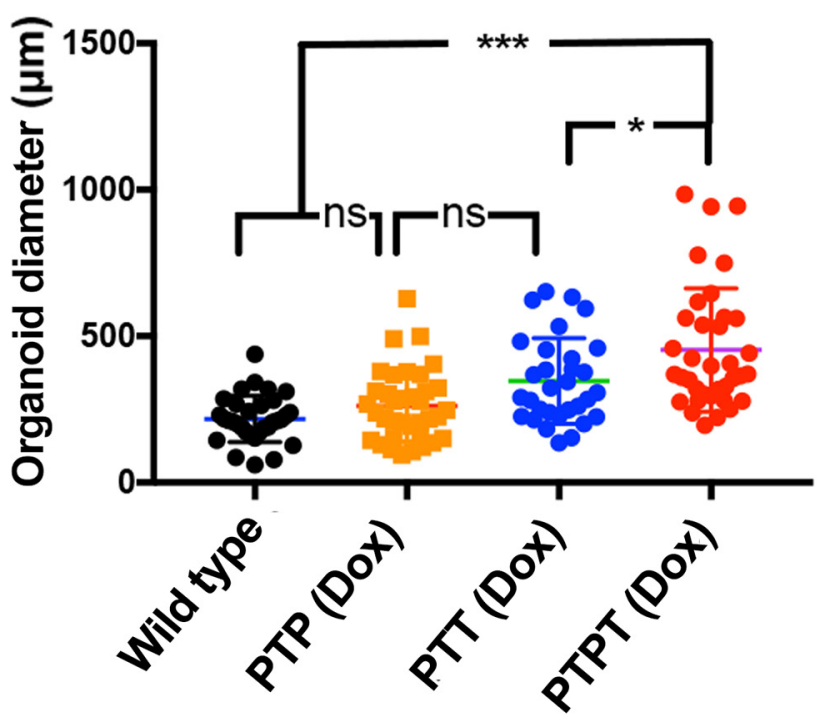

C

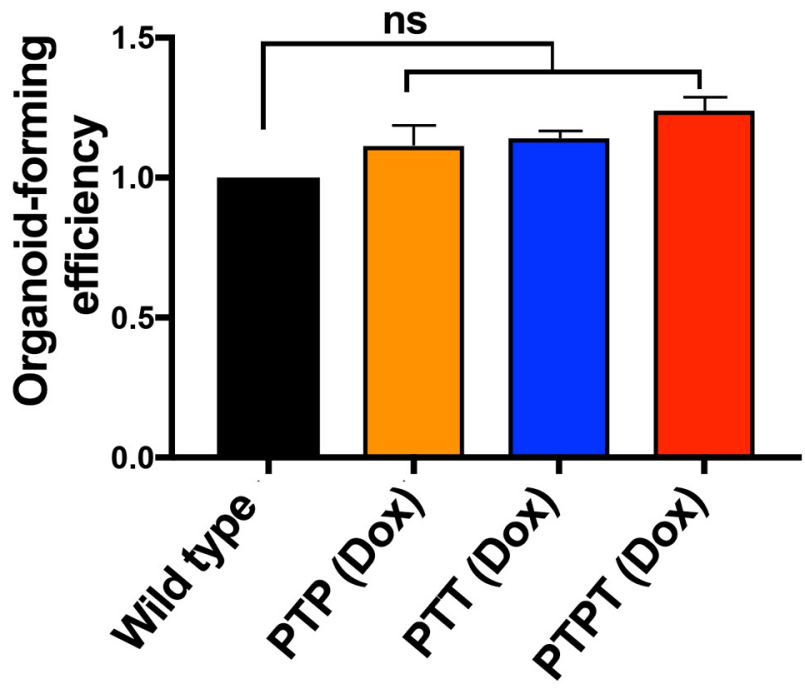


A Superovulation

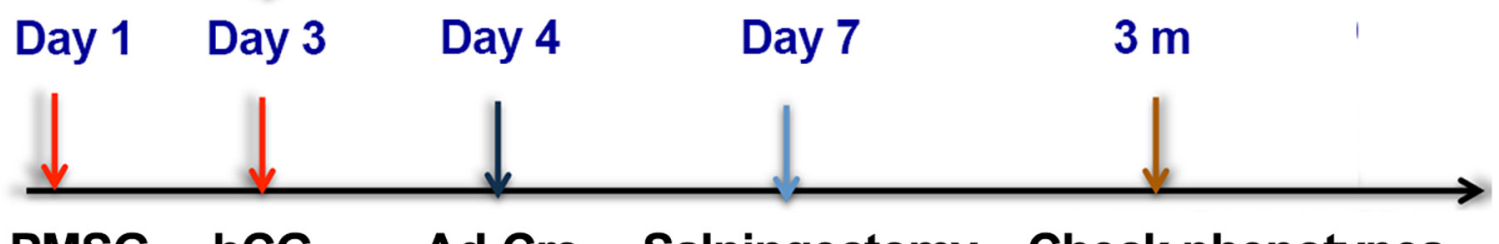

\section{PMSG hCG Ad-Cre Salpingectomy Check phenotypes}

B

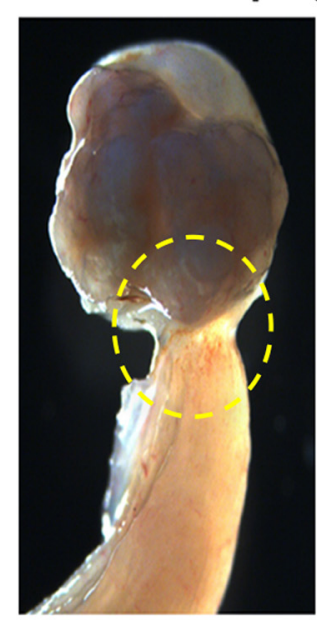

Tp53 ${ }^{\text {R172H/fi }} ;$ T121 (+AdCre)
C Sham control
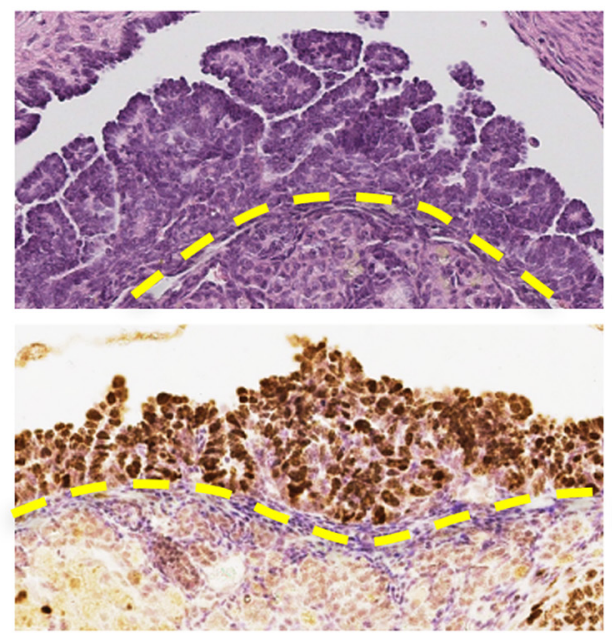

$T p 53^{\text {R172Hff }} ;$ T121 (+AdCre)
Salpingectomy

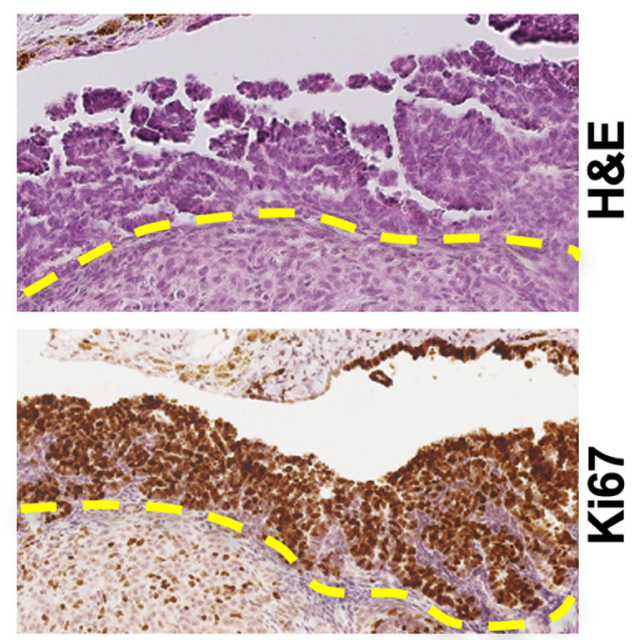

$\frac{1}{2}$

\section{Supplementary Fig.5}


A

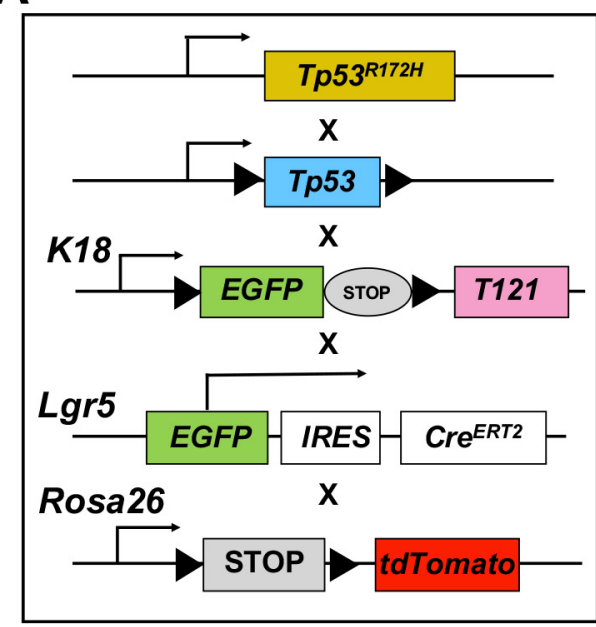

B

4-OHT harvest harvest harvest

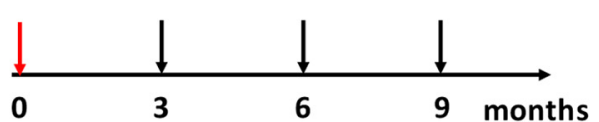

3 month chase

6 month chase

9 month chase

C

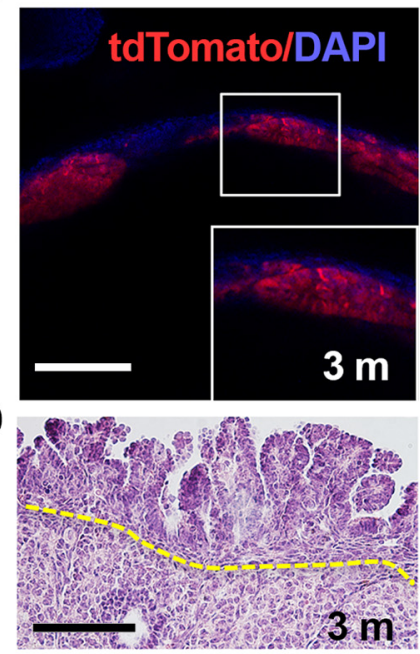

E

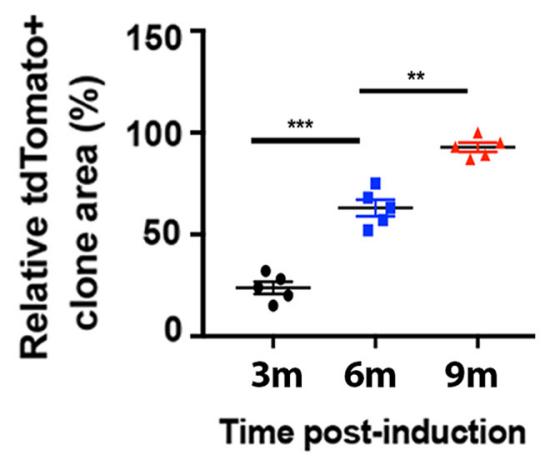

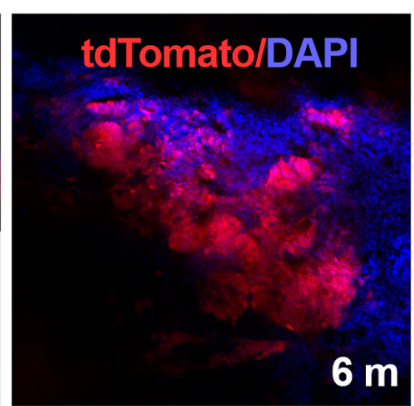
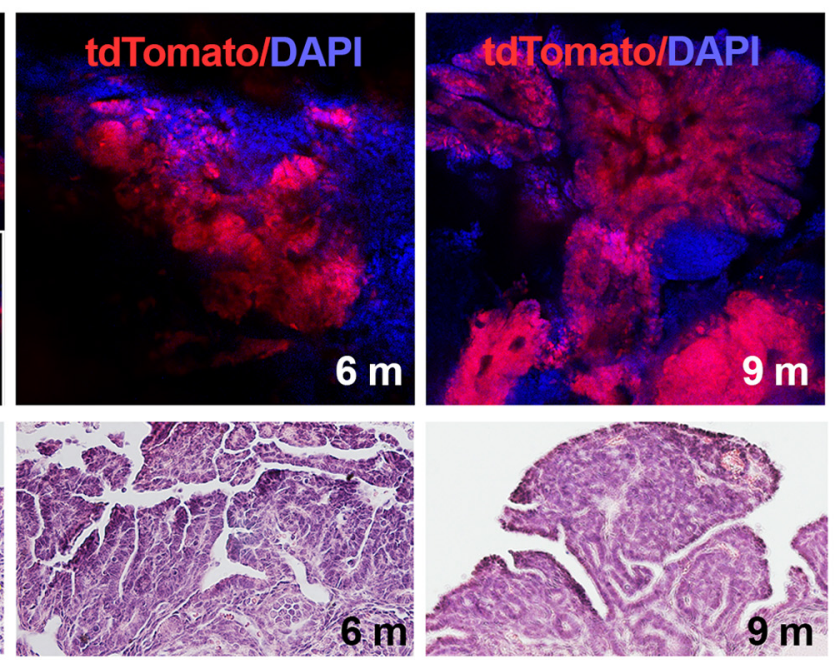

$\mathbf{F}$

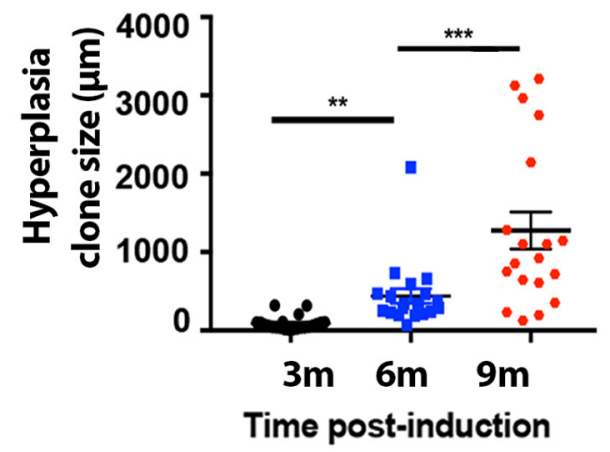

G

without 4-OHT

with 4-OHT
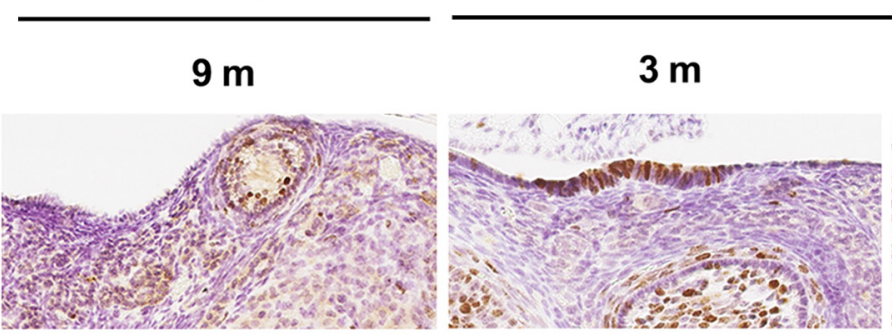

$6 \mathrm{~m}$

$9 \mathrm{~m}$
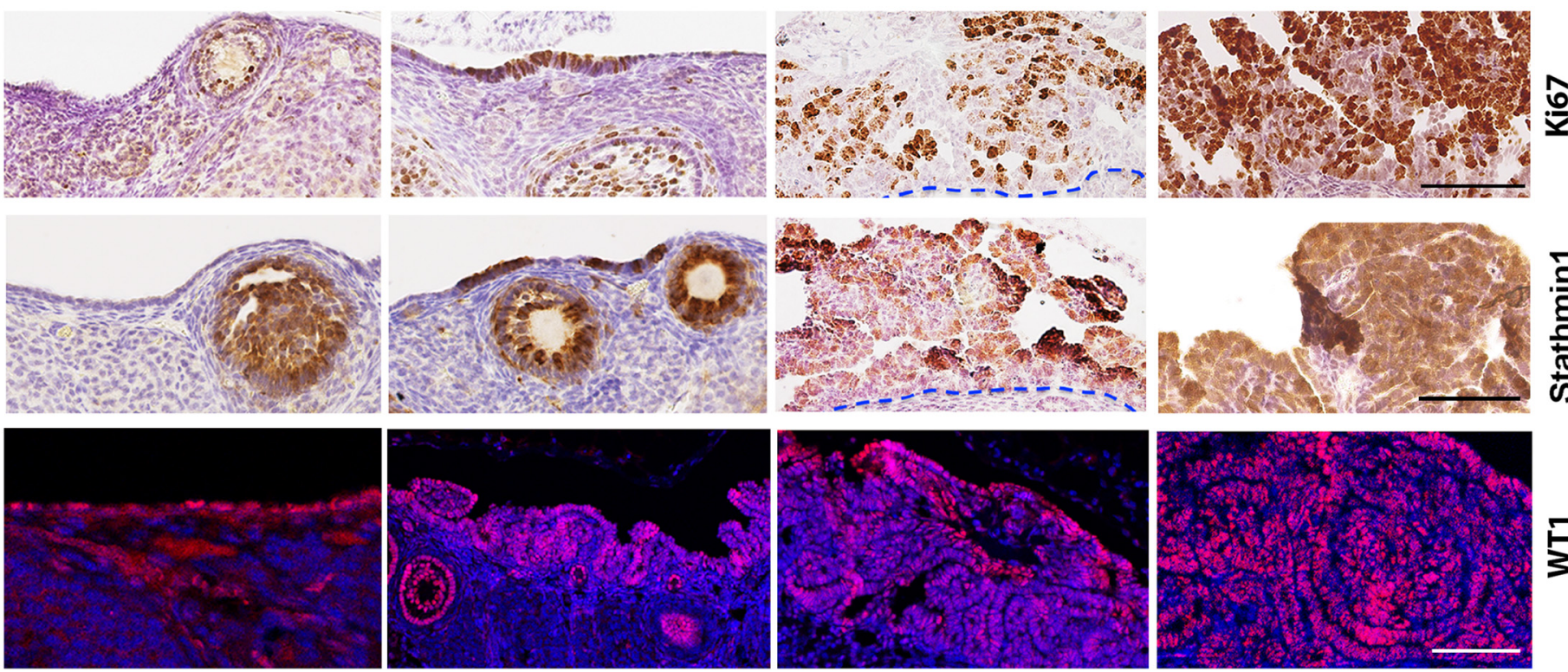

言 
A
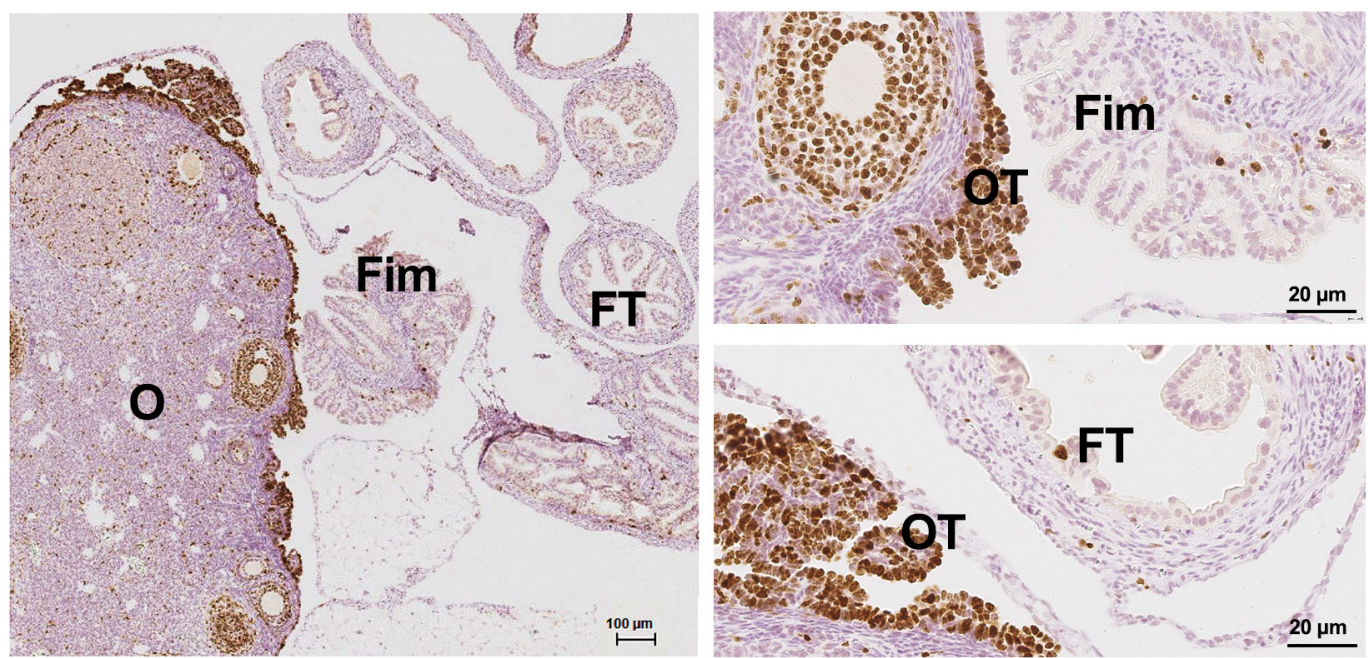

B
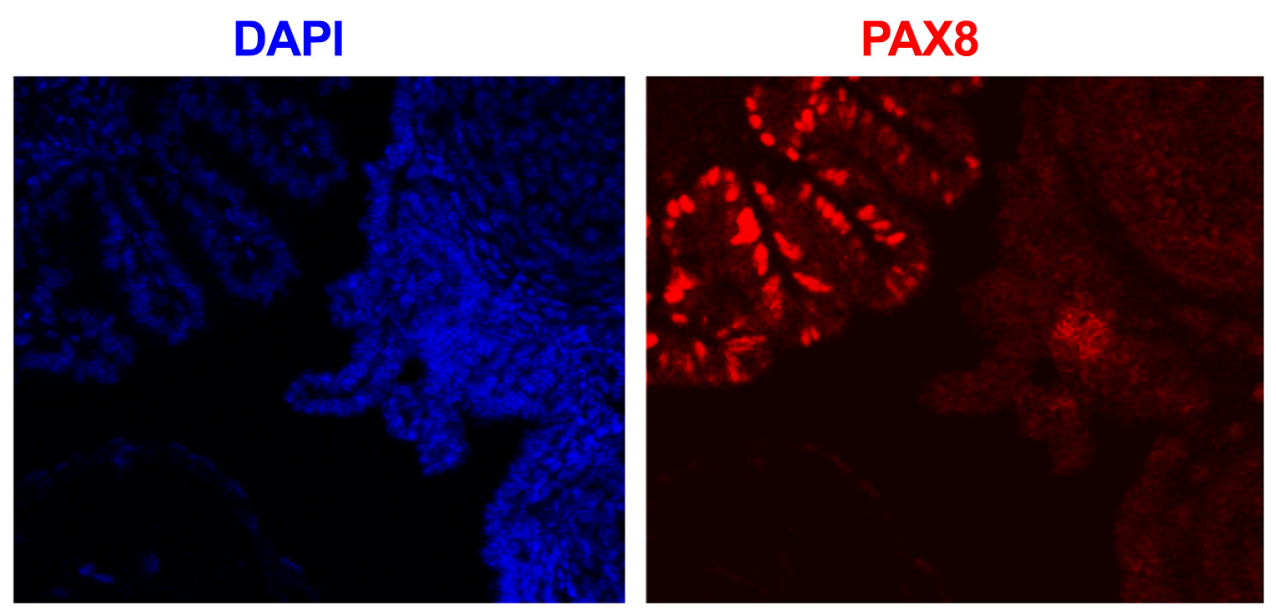

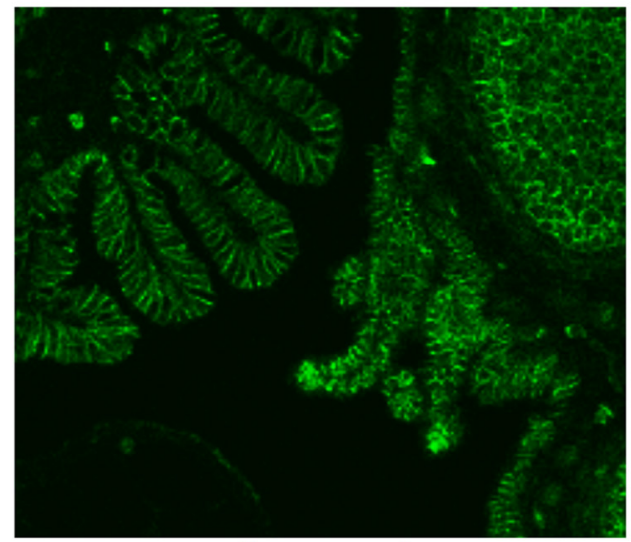

$\beta$-catenin

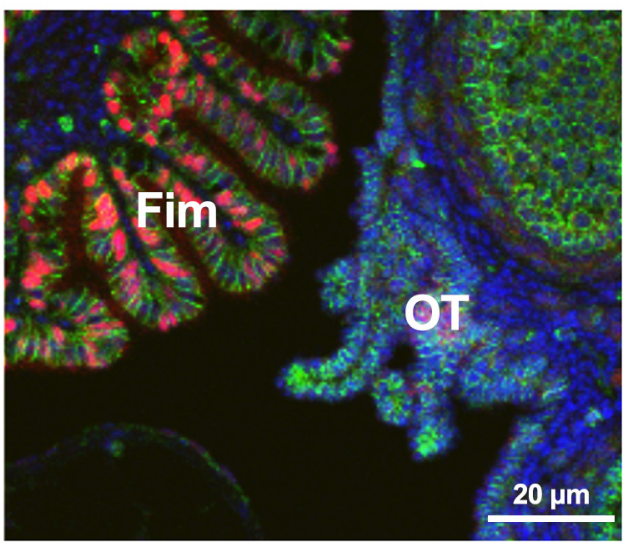

Merge

\section{Supplementary Fig.7}


T-O vs T-FT 50 most significant

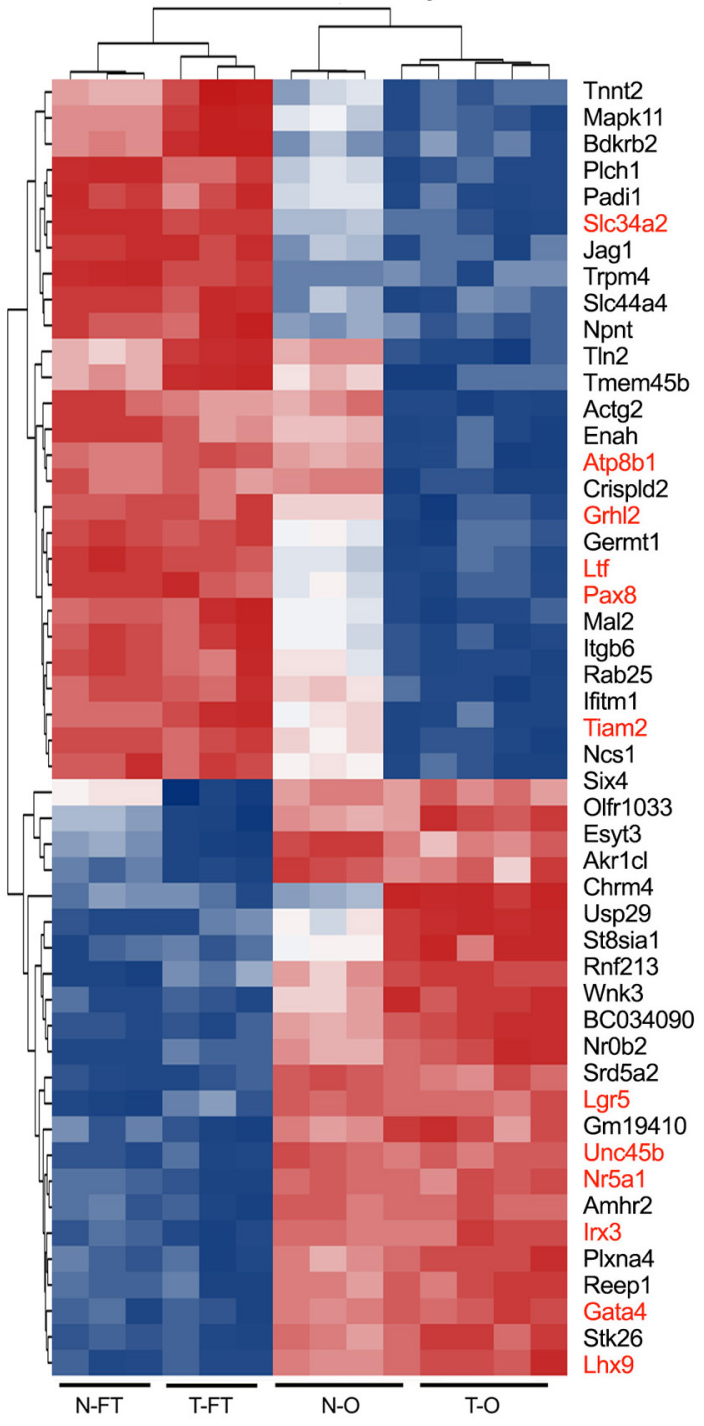

T-O vs T-FT 100 most significant

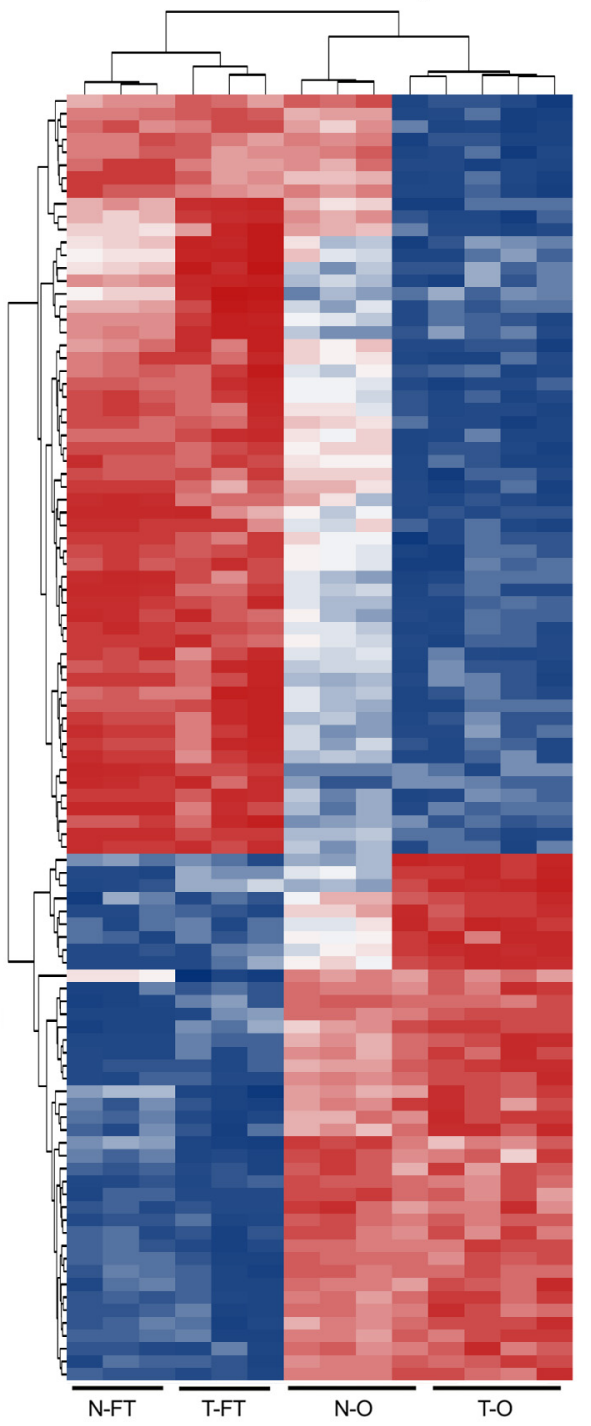

T-O vs T-FT 1000 most significant

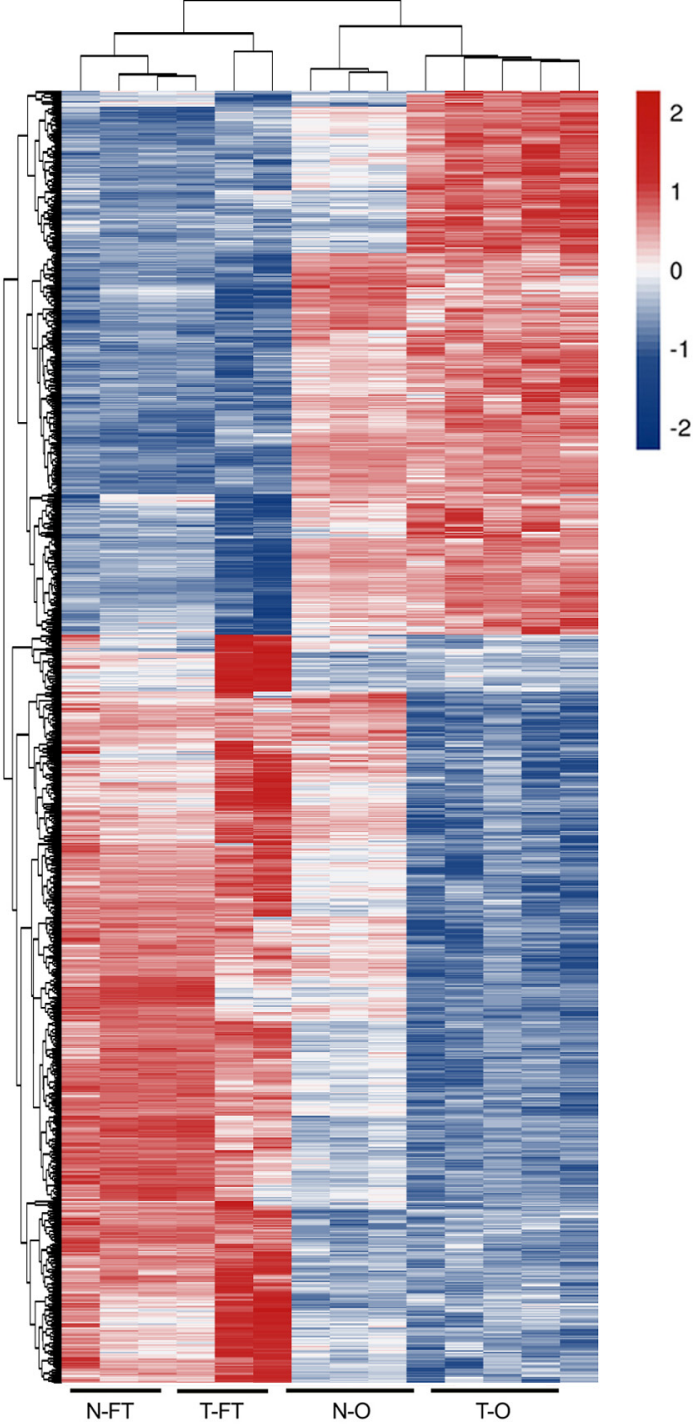

Supplementary Fig.8 
A

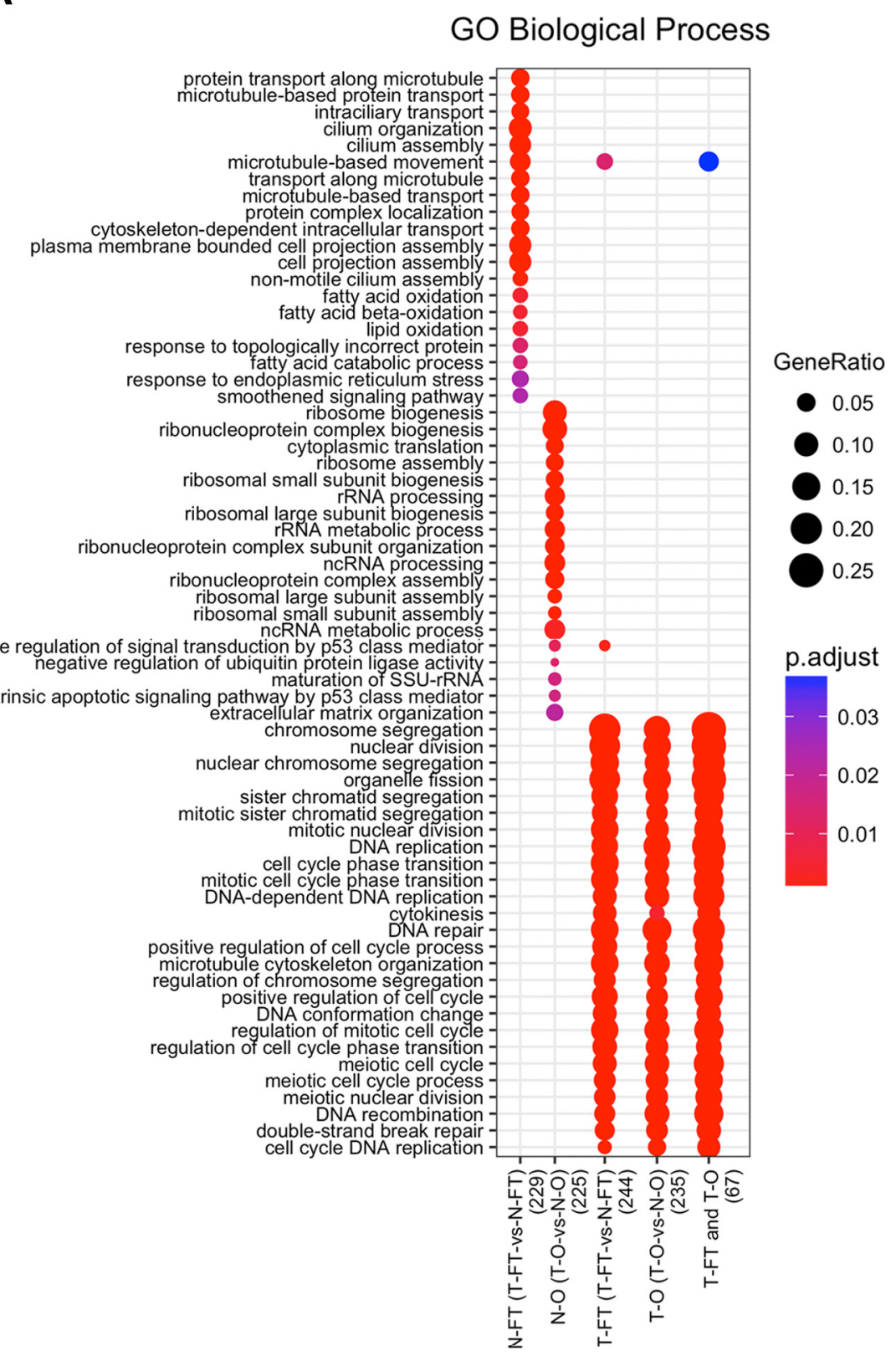

B

\section{GSEA Motif Gene Sets}

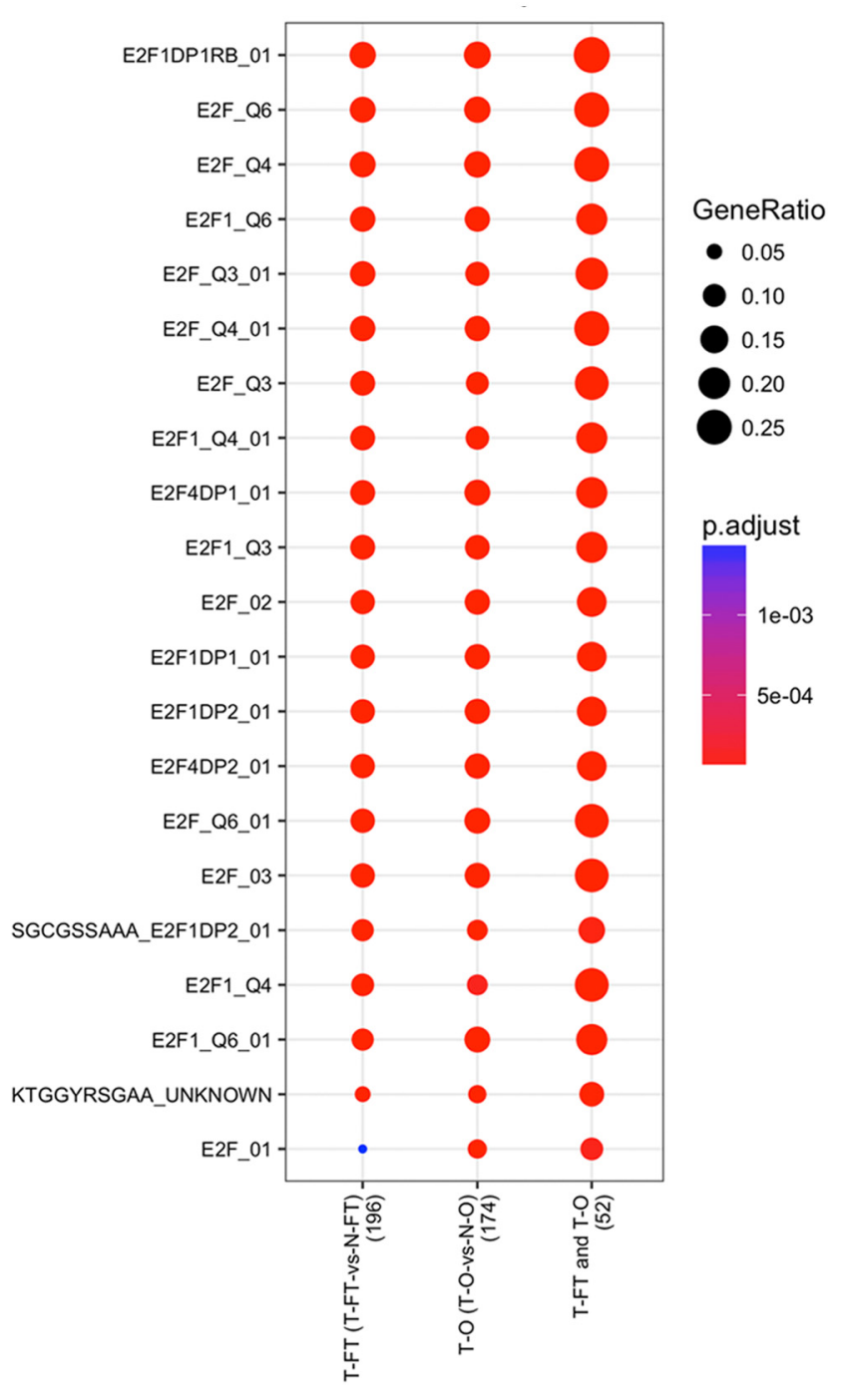

\section{Supplementary Fig.9}



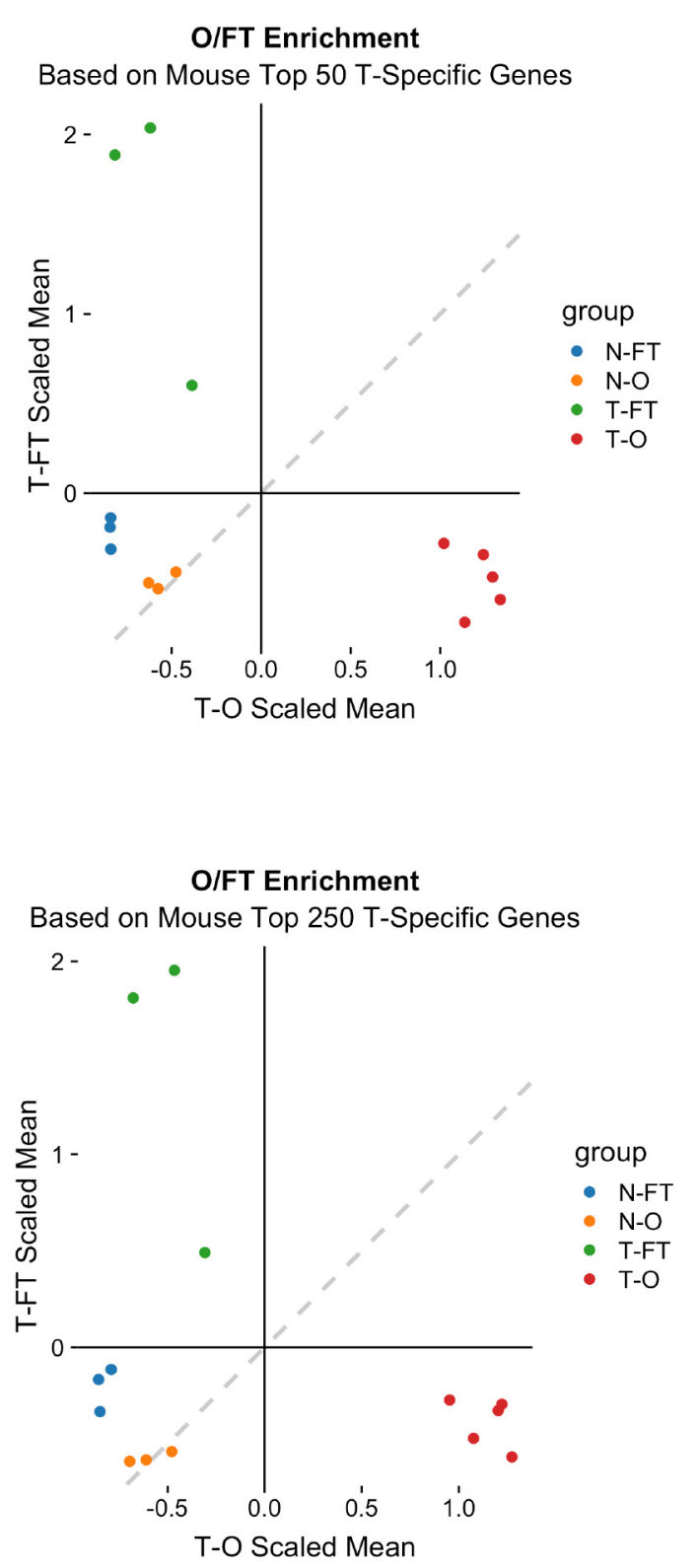
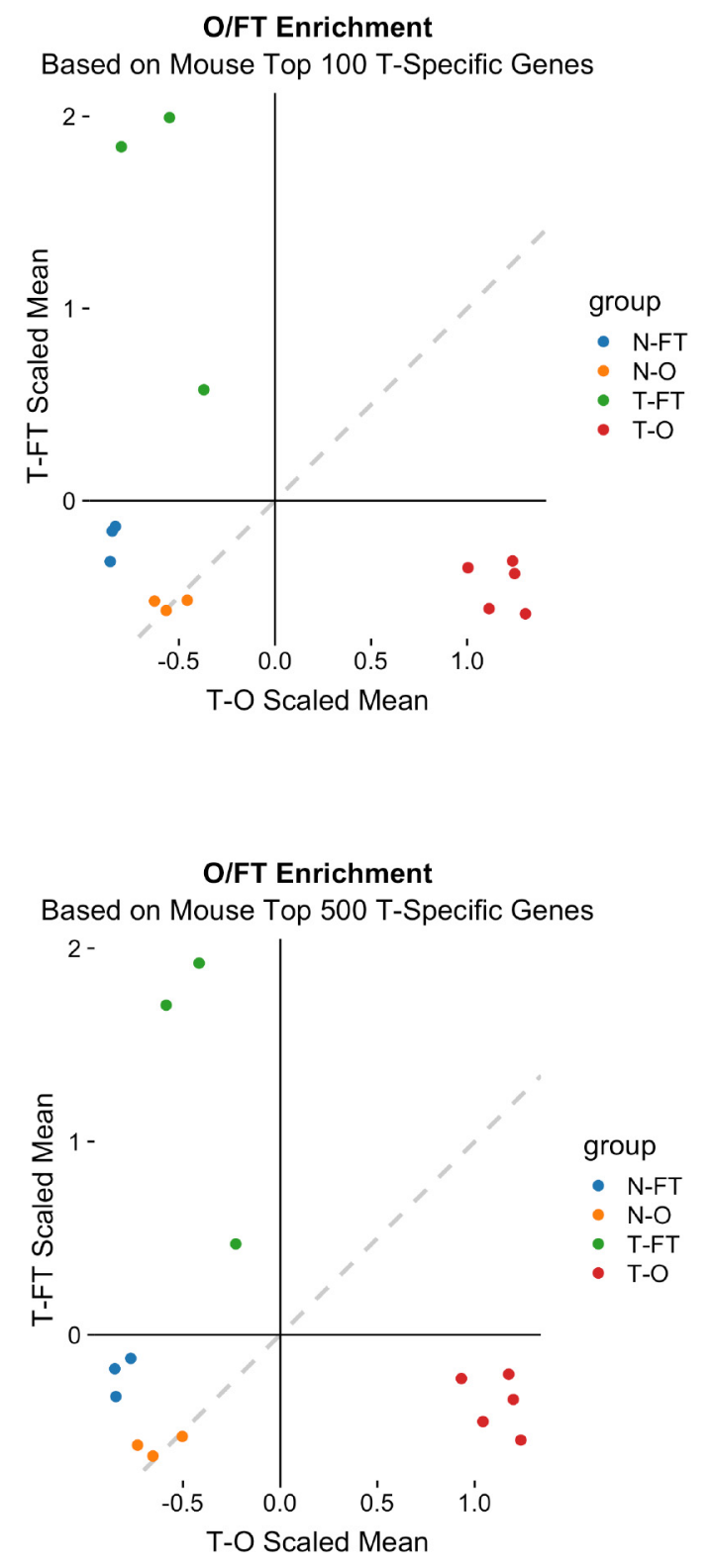

\section{Supplementary Fig. 10}




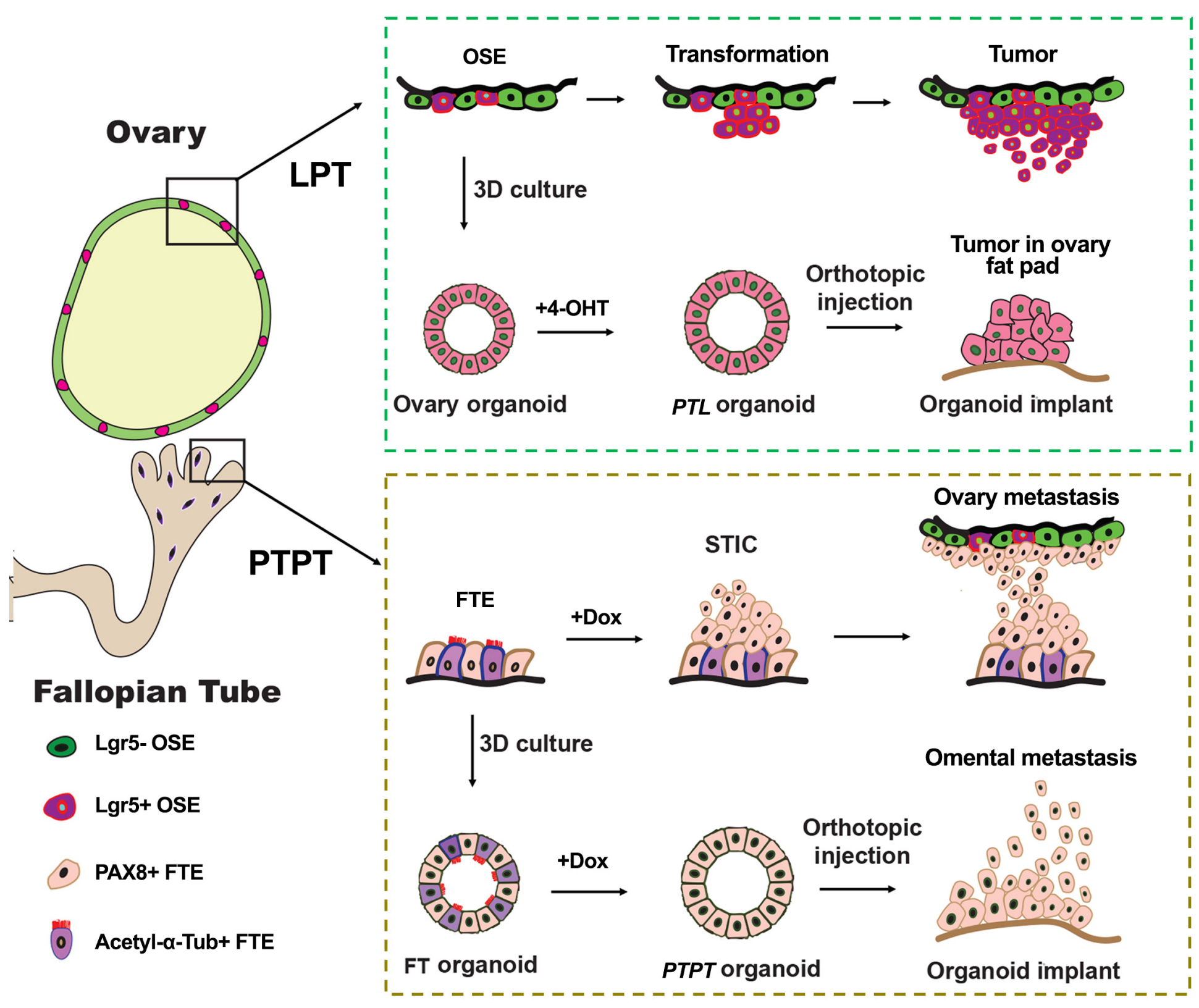

\section{Supplementary Fig.11}

